\title{
Supplementary Material for: \\ Benchmarking the accuracy of the direct random phase approximation and $\sigma$-functionals for NMR shieldings
}

\author{
Michael Glasbrenner ${ }^{1}$, Daniel Graf ${ }^{1}$, and Christian Ochsenfeld ${ }^{1,2}$ \\ ${ }^{1}$ Chair of Theoretical Chemistry, Department of Chemistry, University of Munich \\ (LMU), Butenandtstr. 7, 81377 Munich, Germany \\ ${ }^{2}$ Max Planck Institute for Solid State Research, D-70569 Stuttgart, Germany
}

November 7, 2021 


\section{List of Figures and Tables}

Figure 1: $\quad$ Comparison of different methods for the pcSseg-2 basis set . . . . . . . . . . . . S3

Figure 2: $\quad$ Basis set convergence of the $\mathrm{CCSD}(\mathrm{T})$ shieldings . . . . . . . . . . . . . . . . . . . . S3

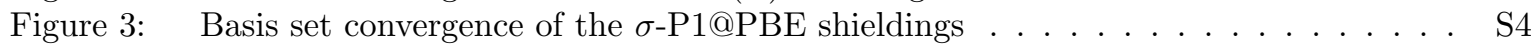

Table 1: $\quad$ Absolute isotropic NMR shieldings in a cc-pVDZ basis set. . . . . . . . . . . . . . S5

Table 2: $\quad$ Statistics for cc-pVDZ-shieldings with CCSD(T)/aug-cc-pCV[TQ]Z as reference. . S6

Table 3: $\quad$ Statistics for cc-pVDZ-shieldings with $\operatorname{CCSD}(\mathrm{T})$ as reference. . . . . . . . . . . . . S6

Table 4: Absolute isotropic NMR shieldings in a cc-pVTZ basis set. . . . . . . . . . . . . . S S7

Table 5: $\quad$ Absolute isotropic NMR shieldings in a cc-pVTZ basis set. . . . . . . . . . . . . S8

Table 6: $\quad$ Statistics for cc-pVTZ-shieldings with CCSD(T)/aug-cc-pCV[TQ]Z as reference. . S9

Table 7: $\quad$ Statistics for cc-pVTZ-shieldings with $\operatorname{CCSD}(\mathrm{T})$ as reference. . . . . . . . . . . . S9

Table 8: Diamagnetic contributions to the NMR shieldings in a cc-pVTZ basis set. . . . . . S10

Table 9: $\quad$ Paramagnetic contributions to the NMR shieldings in a cc-pVTZ basis set. . . . . S11

Table 10: $\quad$ Diamagnetic contributions to the NMR shieldings in a cc-pVTZ basis set. . . . . . S12

Table 11: $\quad$ Paramagnetic contributions to the NMR shieldings in a cc-pVTZ basis set. . . . . S13

Table 12: $\quad$ Testing influence of $\delta$ and DFT grid and gauge-origin independence in a cc-pVTZ

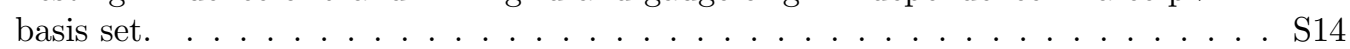

Table 13: Comparison of analytical and numerical shieldings in a cc-pVTZ basis set. . . . . . S15

Table 14: Testing influence of number of frequency integration points in a cc-pVTZ basis set. S16

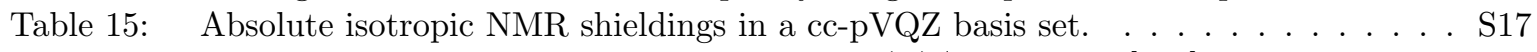

Table 16: $\quad$ Statistics for cc-pVQZ-shieldings with CCSD(T)/aug-cc-pCV[TQ]Z as reference. . S18

Table 17: $\quad$ Statistics for cc-pVQZ-shieldings with $\operatorname{CCSD}(\mathrm{T})$ as reference. . . . . . . . . . . . . S18

Table 18: $\quad$ Absolute isotropic NMR shieldings in a cc-pCVDZ basis set. . . . . . . . . . . . . . S19

Table 19: $\quad$ Statistics for cc-pCVDZ-shieldings with CCSD(T)/aug-cc-pCV[TQ]Z as reference. S20

Table 20: $\quad$ Statistics for cc-pCVDZ-shieldings with CCSD $(\mathrm{T})$ as reference. . . . . . . . . . . . S20

Table 21: $\quad$ Absolute isotropic NMR shieldings in a cc-pCVTZ basis set. $\ldots$. . . . . . . . . . . S21

Table 22: $\quad$ Statistics for cc-pCVTZ-shieldings with CCSD(T)/aug-cc-pCV[TQ]Z as reference. S22

Table 23: $\quad$ Statistics for cc-pCVTZ-shieldings with CCSD $(\mathrm{T})$ as reference. . . . . . . . . . . . S22

Table 24: $\quad$ Absolute isotropic NMR shieldings in a cc-pCVQZ basis set. . . . . . . . . . . . . . S23

Table 25: $\quad$ Statistics for cc-pCVQZ-shieldings with CCSD(T)/aug-cc-pCV[TQ]Z as reference. S24

Table 26: $\quad$ Statistics for cc-pCVQZ-shieldings with CCSD $(\mathrm{T})$ as reference. . . . . . . . . . . . . S24

Table 27: $\quad$ Absolute isotropic NMR shieldings in a def2-SVP basis set. . . . . . . . . . . . . S25

Table 28: $\quad$ Statistics for def2-SVP-shieldings with CCSD(T)/aug-cc-pCV[TQ]Z as reference. . S26

Table 29: $\quad$ Statistics for def2-SVP-shieldings with CCSD $(\mathrm{T})$ as reference. . . . . . . . . . . . . S26

Table 30: $\quad$ Absolute isotropic NMR shieldings in a def2-TZVP basis set. . . . . . . . . . . . . . S27

Table 31: $\quad$ Statistics for def2-TZVP-shieldings with CCSD(T)/aug-cc-pCV[TQ]Z as reference. S28

Table 32: $\quad$ Statistics for def2-TZVP-shieldings with CCSD $(\mathrm{T})$ as reference. . . . . . . . . . . . S28

Table 33: $\quad$ Absolute isotropic NMR shieldings in a def2-QZVP basis set. . . . . . . . . . . . . . . S29

Table 34: $\quad$ Statistics for def2-QZVP-shieldings with CCSD(T)/aug-cc-pCV[TQ]Z as reference. S30

Table 35: $\quad$ Statistics for def2-QZVP-shieldings with CCSD $(\mathrm{T})$ as reference. . . . . . . . . . . . S30

Table 36: Absolute isotropic NMR shieldings in a pcSseg-0 basis set. . . . . . . . . . . . . . . S31

Table 37: $\quad$ Statistics for pcSseg-0-shieldings with CCSD(T)/aug-cc-pCV[TQ]Z as reference. . S32

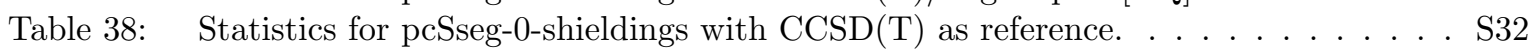

Table 39: $\quad$ Testing influence of the auxiliary basis set in a pcSseg-0 basis set. . . . . . . . . . S33

Table 40: $\quad$ Absolute isotropic NMR shieldings in a pcSseg-1 basis set. . . . . . . . . . . . . . . S34

Table 41: $\quad$ Statistics for pcSseg-1-shieldings with CCSD(T)/aug-cc-pCV[TQ]Z as reference. . S35

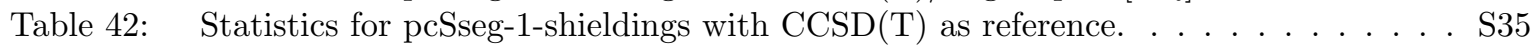

Table 43: $\quad$ Absolute isotropic NMR shieldings in a pcSseg-2 basis set. . . . . . . . . . . . . . . S36

Table 44: Absolute isotropic NMR shieldings in a pcSseg-2 basis set. . . . . . . . . . . . . . . S37

Table 45: $\quad$ Statistics for pcSseg-2-shieldings with CCSD $(\mathrm{T}) /$ aug-cc-pCV[TQ]Z as reference. . S38

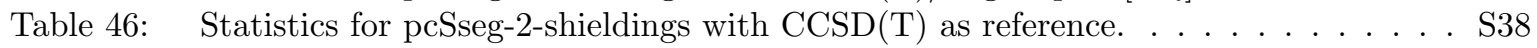




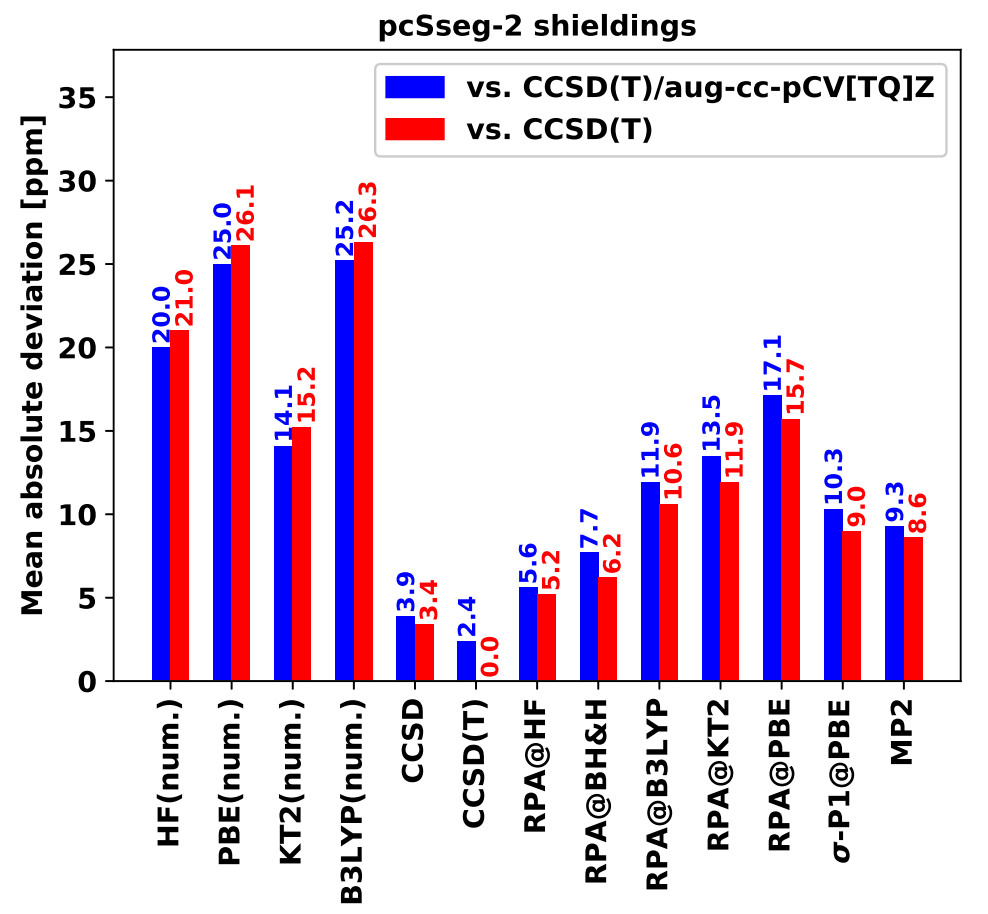

Figure 1: Mean absolute deviations of NMR-shieldings on the employed test set using the pcSseg-2 basis set in all calculations. The blue bars display the deviation from basis-set extrapolated (aug-cc-pCV[TQ]Z) $\operatorname{CCSD}(\mathrm{T})$-shieldings; the red bars display deviations from $\operatorname{CCSD}(\mathrm{T})$-shieldings obtained with a pcSseg-2 basis set. "(num.)" denotes shieldings computed with a numerical derivative.

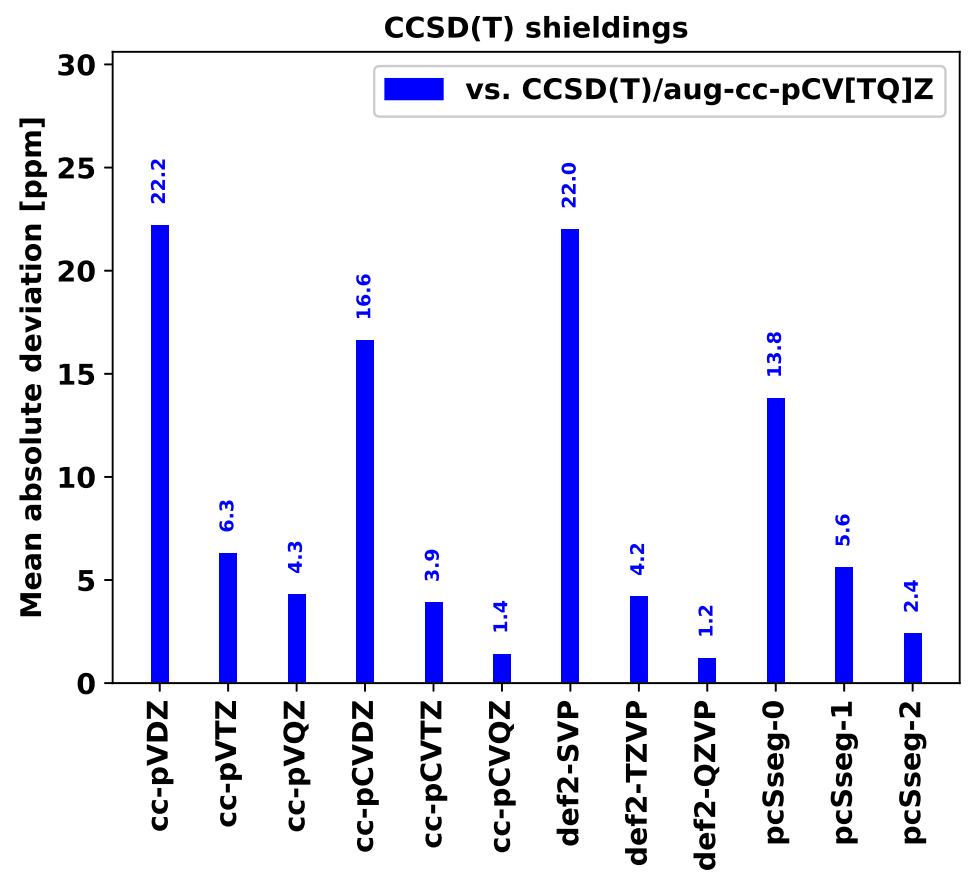

Figure 2: Mean absolute deviations of $\operatorname{CCSD}(\mathrm{T})$ shieldings using different basis sets. Basis-set extrapolated (aug-cc-pCV[TQ]Z) CCSD(T)-shieldings are used as a reference. All $\operatorname{CCSD}(\mathrm{T})$ shielding calculations were done with the CFOUR program [1, 2]. 


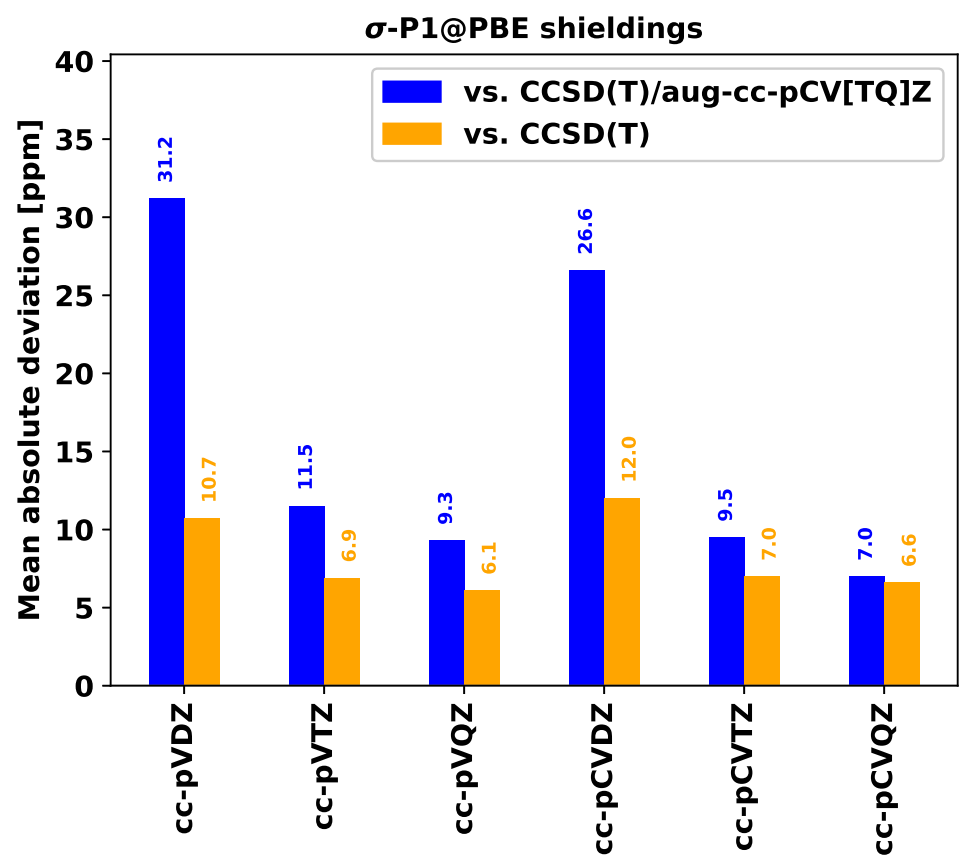

Figure 3: Mean absolute deviations of $\sigma$-P1@PBE shieldings using different basis sets. Basis-set extrapolated (aug-cc-pCV[TQ]Z) CCSD $(\mathrm{T})$-shieldings and $\operatorname{CCSD}(\mathrm{T})$ with the same respective basis set are used as references. All $\operatorname{CCSD}(\mathrm{T})$ shielding calculations were done with the CFOUR program [1, 2]. 
Table 1: Absolute isotropic NMR shieldings in a cc-pVDZ basis. All values are given in ppm. "Extrap." stands for basis-set extrapolated $\operatorname{CCSD}(\mathrm{T})$-shieldings in an aug-cc-pCV[TQ]Z basis set. The data for "Extrap." was taken from Ref. 3. CCSD(T) calculations were done with the CFOUR program[1, 2.

\begin{tabular}{|c|c|c|c|c|c|c|}
\hline Mol. & Nuc. & $\overline{\mathrm{CCSD}(\mathrm{T})}$ & Extrap. & $\overline{\mathrm{RPA} @ H F}$ & RPA@PBE & $\sigma-\mathrm{P} 1 @ \mathrm{PBE}$ \\
\hline \multirow[t]{2}{*}{$\mathrm{HF}$} & $\mathrm{H}$ & 30.5 & 28.8 & 30.4 & 31.1 & 31.4 \\
\hline & $\mathrm{F}$ & 427.0 & 420.3 & 427.2 & 425.1 & 422.0 \\
\hline \multirow[t]{2}{*}{$\mathrm{CO}$} & $\mathrm{C}$ & 34.3 & 2.2 & 32.2 & 55.8 & 46.9 \\
\hline & $\mathrm{O}$ & -16.5 & -55.0 & -10.2 & 18.6 & 0.1 \\
\hline $\mathrm{N}_{2}$ & $\mathrm{~N}$ & -25.6 & -60.4 & -25.4 & 3.7 & -11.8 \\
\hline \multirow[t]{2}{*}{$\mathrm{H}_{2} \mathrm{O}$} & $\mathrm{O}$ & 354.9 & 338.0 & 353.8 & 351.8 & 347.9 \\
\hline & $\mathrm{H}$ & 31.9 & 30.6 & 31.9 & 32.3 & 32.5 \\
\hline \multirow[t]{3}{*}{$\mathrm{HCN}$} & $\mathrm{H}$ & 29.4 & 29.0 & 29.6 & 29.6 & 29.7 \\
\hline & $\mathrm{C}$ & 111.5 & 84.6 & 108.7 & 120.1 & 117.4 \\
\hline & $\mathrm{N}$ & 21.5 & -14.1 & 20.3 & 45.0 & 37.9 \\
\hline \multirow[t]{3}{*}{$\mathrm{HOF}$} & $\mathrm{O}$ & -5.6 & -68.9 & 9.1 & 76.6 & 43.1 \\
\hline & $\mathrm{H}$ & 21.8 & 19.6 & 22.6 & 24.5 & 23.8 \\
\hline & $\mathrm{F}$ & 183.1 & 192.2 & 206.6 & 222.0 & 213.2 \\
\hline \multirow[t]{2}{*}{$\mathrm{NH}_{3}$} & $\mathrm{~N}$ & 283.9 & 270.7 & 281.8 & 281.3 & 279.0 \\
\hline & $\mathrm{H}$ & 32.3 & 31.4 & 32.4 & 32.5 & 32.6 \\
\hline \multirow[t]{3}{*}{$\mathrm{CH}_{2} \mathrm{O}$} & $\mathrm{O}$ & -339.3 & -378.6 & -309.5 & -204.3 & -259.4 \\
\hline & $\mathrm{C}$ & 36.9 & 1.5 & 37.1 & 59.3 & 54.2 \\
\hline & $\mathrm{H}$ & 22.7 & 22.0 & 23.1 & 23.7 & 23.4 \\
\hline \multirow[t]{2}{*}{$\mathrm{CH}_{4}$} & $\mathrm{C}$ & 209.6 & 198.9 & 207.6 & 208.9 & 207.2 \\
\hline & $\mathrm{H}$ & 31.6 & 31.3 & 31.7 & 31.7 & 31.7 \\
\hline \multirow{2}{*}{$\mathrm{C} 2 \mathrm{H} 4$} & $\mathrm{C}$ & 95.6 & 69.7 & 92.3 & 103.4 & 102.7 \\
\hline & $\mathrm{H}$ & 26.6 & 26.0 & 26.7 & 27.0 & 27.0 \\
\hline \multirow[t]{2}{*}{$\mathrm{AlF}$} & $\mathrm{Al}$ & 599.3 & 572.9 & 604.1 & 610.6 & 607.0 \\
\hline & $\mathrm{F}$ & 190.9 & 211.8 & 212.3 & 207.8 & 204.7 \\
\hline \multirow[t]{3}{*}{$\mathrm{CH}_{3} \mathrm{~F}$} & $\mathrm{C}$ & 142.9 & 122.1 & 142.9 & 147.2 & 146.0 \\
\hline & $\mathrm{F}$ & 475.9 & 482.9 & 476.7 & 470.5 & 471.0 \\
\hline & $\mathrm{H}$ & 27.9 & 27.3 & 28.1 & 28.2 & 28.3 \\
\hline $\mathrm{C}_{3} \mathrm{H}_{4}$ & $\mathrm{C}$ & 205.0 & 192.1 & 203.9 & 205.6 & 205.4 \\
\hline & $\mathrm{C}$ & 107.5 & 83.7 & 105.6 & 115.5 & 113.9 \\
\hline & $\mathrm{H}$ & 25.1 & 24.4 & 25.2 & 25.6 & 25.5 \\
\hline & $\mathrm{H}$ & 31.2 & 30.6 & 31.3 & 31.3 & 31.4 \\
\hline $\mathrm{FCCH}$ & $\mathrm{C}$ & 192.5 & 179.9 & 190.7 & 194.5 & 195.6 \\
\hline & $\mathrm{C}$ & 125.5 & 100.1 & 125.0 & 129.5 & 129.2 \\
\hline & $\mathrm{H}$ & 31.0 & 30.5 & 31.1 & 31.2 & 31.3 \\
\hline & $\mathrm{F}$ & 429.6 & 423.5 & 433.1 & 431.2 & 431.9 \\
\hline $\mathrm{FCN}$ & $\mathrm{F}$ & 385.1 & 374.1 & 392.3 & 391.3 & 387.2 \\
\hline & $\mathrm{C}$ & 110.7 & 82.2 & 108.5 & 116.0 & 114.6 \\
\hline & $\mathrm{N}$ & 140.5 & 117.9 & 137.8 & 153.0 & 151.2 \\
\hline $\mathrm{H}_{2} \mathrm{~S}$ & $\mathrm{~S}$ & 783.0 & 739.0 & 776.4 & 785.2 & 768.2 \\
\hline & $\mathrm{H}$ & 31.4 & 30.5 & 31.5 & 31.7 & 31.9 \\
\hline HCP & $\mathrm{H}$ & 29.4 & 29.6 & 29.7 & 29.6 & 29.7 \\
\hline & $\mathrm{C}$ & 67.3 & 37.6 & 61.2 & 78.4 & 76.6 \\
\hline & $\mathrm{P}$ & 471.7 & 388.0 & 456.1 & 510.3 & 499.6 \\
\hline $\mathrm{HFCO}$ & $\mathrm{O}$ & -58.6 & -94.3 & -48.5 & -15.6 & -33.6 \\
\hline & $\mathrm{C}$ & 68.0 & 39.6 & 66.9 & 76.7 & 74.3 \\
\hline & $\mathrm{F}$ & 187.0 & 165.3 & 204.5 & 207.1 & 196.6 \\
\hline & $\mathrm{H}$ & 24.7 & 23.9 & 24.9 & 25.0 & 24.9 \\
\hline $\mathrm{H}_{2} \mathrm{C}_{2} \mathrm{O}$ & $\mathrm{C}$ & 205.3 & 193.3 & 204.4 & 205.8 & 205.2 \\
\hline & $\mathrm{C}$ & 26.1 & -6.3 & 26.5 & 37.5 & 31.0 \\
\hline & $\mathrm{O}$ & 22.2 & -5.9 & 36.3 & 51.0 & 35.1 \\
\hline & $\mathrm{H}$ & 30.0 & 29.2 & 30.2 & 30.3 & 30.4 \\
\hline $\mathrm{LiF}$ & $\mathrm{Li}$ & 94.4 & 89.3 & 94.5 & 94.6 & 94.6 \\
\hline & $\mathrm{F}$ & 350.0 & 382.5 & 359.8 & 352.2 & 351.4 \\
\hline $\mathrm{LiH}$ & $\mathrm{H}$ & 26.3 & 26.6 & 26.5 & 26.2 & 26.3 \\
\hline & $\mathrm{Li}$ & 92.4 & 89.3 & 92.4 & 93.7 & 93.6 \\
\hline $\mathrm{N}_{2} \mathrm{O}$ & $\mathrm{N}$ & 129.6 & 106.4 & 127.9 & 144.2 & 139.8 \\
\hline & $\mathrm{N}$ & 45.4 & 12.6 & 41.0 & 60.3 & 55.0 \\
\hline & $\mathrm{O}$ & 209.8 & 199.0 & 215.9 & 223.7 & 217.2 \\
\hline OCS & $\mathrm{O}$ & 123.0 & 96.8 & 128.1 & 140.9 & 132.8 \\
\hline & $\mathrm{C}$ & 58.3 & 30.2 & 54.6 & 69.5 & 66.4 \\
\hline & $\mathrm{S}$ & 840.0 & 796.7 & 845.6 & 857.4 & 848.2 \\
\hline $\mathrm{OF}_{2}$ & $\mathrm{O}$ & -339.7 & -447.1 & -306.1 & -130.0 & -229.2 \\
\hline & $\mathrm{F}$ & 18.7 & -24.0 & 49.5 & 79.9 & 58.4 \\
\hline $\mathrm{H}_{4} \mathrm{C}_{2} \mathrm{O}$ & $\mathrm{O}$ & 364.8 & 363.2 & 369.5 & 364.7 & 364.2 \\
\hline & $\mathrm{C}$ & 170.7 & 153.2 & 170.6 & 173.5 & 173.0 \\
\hline & $\mathrm{H}$ & 29.7 & 29.1 & 30.0 & 30.0 & 30.0 \\
\hline $\mathrm{PN}$ & $\mathrm{N}$ & -282.0 & -344.0 & -273.9 & -192.3 & -245.0 \\
\hline & $\mathrm{P}$ & 189.0 & 50.6 & 176.9 & 302.7 & 224.5 \\
\hline
\end{tabular}


Table 2: Statistics for absolute isotropic NMR shieldings in a cc-pVDZ basis. CCSD(T)/aug-cc$\mathrm{pCV}[\mathrm{TQ}] \mathrm{Z}$ shieldings are used as a reference. $\mathrm{MSD}=$ mean signed deviation; $\mathrm{MAD}=$ mean absolute deviation; $\mathrm{MRD}=$ mean relative deviation [\%]; $\mathrm{MARD}=$ mean absolute relative deviation [\%]; $\mathrm{RMSD}=$ root mean squared deviation.

\begin{tabular}{lrrrrr}
\hline \hline & MSD & MAD & MRD & MARD & RMSD \\
\hline RHF & 1.6 & 15.8 & 8.9 & 39.5 & 28.7 \\
CCSD & 20.0 & 21.3 & 92.4 & 92.8 & 32.4 \\
CCSD(T) & 20.2 & 22.2 & 98.7 & 99.5 & 33.4 \\
RPA@HF & 22.9 & 23.7 & 103.0 & 103.2 & 36.4 \\
RPA@PBE & 37.9 & 39.3 & 165.9 & 166.3 & 69.2 \\
$\sigma-P 1 @ P B E$ & 29.7 & 31.2 & 140.8 & 141.2 & 51.0 \\
MP2 & 28.6 & 30.5 & 126.1 & 126.9 & 48.3 \\
\hline \hline
\end{tabular}

Table 3: Statistics for absolute isotropic NMR shieldings in a cc-pVDZ basis. CCSD(T) shieldings are used as a reference. $\mathrm{MSD}=$ mean signed deviation; $\mathrm{MAD}=$ mean absolute deviation; $\mathrm{MRD}=$ mean relative deviation [\%]; MARD=mean absolute relative deviation [\%]; RMSD=root mean squared deviation.

\begin{tabular}{lrrrrr}
\hline \hline & MSD & MAD & MRD & MARD & RMSD \\
\hline RHF & -18.5 & 24.8 & -47.3 & 54.5 & 46.6 \\
CCSD & -0.1 & 2.8 & -0.1 & 3.9 & 5.4 \\
CCSD(T) & 0.0 & 0.0 & 0.0 & 0.0 & 0.0 \\
RPA@HF & 2.7 & 5.0 & 8.3 & 10.3 & 9.2 \\
RPA@PBE & 17.7 & 18.1 & 44.7 & 44.8 & 39.5 \\
$\sigma$-P1@PBE & 9.6 & 10.7 & 26.2 & 26.5 & 21.2 \\
MP2 & 8.4 & 9.0 & 16.8 & 17.3 & 19.1 \\
\hline \hline
\end{tabular}


Table 4: Absolute isotropic NMR shieldings in a cc-pVTZ basis. All values are given in ppm. "Extrap." stands for basis-set extrapolated $\operatorname{CCSD}(\mathrm{T})$-shieldings in an aug-cc-pCV[TQ]Z basis set. The data for "Extrap." was taken from Ref. 3, CCSD(T) calculations were done with the CFOUR program [1, 2.

\begin{tabular}{|c|c|c|c|c|c|c|c|c|c|}
\hline Mol. & Nuc. & $\overline{C \operatorname{CSS}(\mathrm{T})}$ & Extrap. & $\overline{\mathrm{RPA} @ \mathrm{HF}}$ & RPA@PBE & 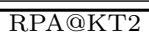 & RPA@B3LYP & RPA@BH\&H & RPA@B97-2 \\
\hline \multirow[t]{2}{*}{$\mathrm{HF}$} & $\mathrm{H}$ & 29.5 & 28.8 & 29.4 & 30.1 & 30.1 & 29.9 & 29.7 & 29.9 \\
\hline & $\mathrm{F}$ & 419.8 & 420.3 & 419.0 & 417.9 & 418.2 & 418.3 & 418.6 & 418.0 \\
\hline \multirow[t]{2}{*}{$\mathrm{CO}$} & $\mathrm{C}$ & 11.3 & 2.2 & 5.6 & 28.4 & 23.6 & 21.5 & 14.3 & 19.7 \\
\hline & $\mathrm{O}$ & -49.8 & -55.0 & -48.4 & -21.4 & -26.2 & -29.7 & -37.8 & -31.7 \\
\hline $\mathrm{N}_{2}$ & $\mathrm{~N}$ & -52.5 & -60.4 & -58.2 & -28.8 & -34.7 & -37.8 & -47.3 & -39.8 \\
\hline \multirow[t]{2}{*}{$\mathrm{H}_{2} \mathrm{O}$} & $\mathrm{O}$ & 344.3 & 338.0 & 341.7 & 341.1 & 341.6 & 341.5 & 341.5 & 341.0 \\
\hline & $\mathrm{H}$ & 31.3 & 30.6 & 31.3 & 31.7 & 31.7 & 31.6 & 31.5 & 31.6 \\
\hline \multirow[t]{3}{*}{$\mathrm{HCN}$} & $\mathrm{H}$ & 29.2 & 29.0 & 29.5 & 29.5 & 29.5 & 29.5 & 29.5 & 29.5 \\
\hline & $\mathrm{C}$ & 91.5 & 84.6 & 87.5 & 98.7 & 96.4 & 95.7 & 92.2 & 94.8 \\
\hline & $\mathrm{N}$ & -7.4 & -14.1 & -11.8 & 12.5 & 6.6 & 6.5 & -1.3 & 3.8 \\
\hline \multirow[t]{3}{*}{$\mathrm{HOF}$} & $\mathrm{O}$ & -44.4 & -68.9 & -40.6 & 24.1 & 0.4 & -5.2 & -26.7 & -10.5 \\
\hline & $\mathrm{H}$ & 20.7 & 19.6 & 21.3 & 22.9 & 22.3 & 22.0 & 21.5 & 22.0 \\
\hline & $\mathrm{F}$ & 188.3 & 192.2 & 212.3 & 213.7 & 210.5 & 210.2 & 209.9 & 210.0 \\
\hline \multirow[t]{2}{*}{$\mathrm{NH}_{3}$} & $\mathrm{~N}$ & 276.2 & 270.7 & 272.8 & 273.0 & 273.3 & 273.3 & 273.0 & 272.6 \\
\hline & $\mathrm{H}$ & 32.0 & 31.4 & 32.1 & 32.2 & 32.2 & 32.2 & 32.2 & 32.2 \\
\hline \multirow[t]{3}{*}{$\mathrm{CH}_{2} \mathrm{O}$} & $\mathrm{O}$ & -376.5 & -378.6 & -351.6 & -266.4 & -292.3 & -293.5 & -316.4 & -302.2 \\
\hline & $\mathrm{C}$ & 13.2 & 1.5 & 12.3 & 31.6 & 25.4 & 24.8 & 18.6 & 22.9 \\
\hline & $\mathrm{H}$ & 22.3 & 22.0 & 22.9 & 23.3 & 23.1 & 23.0 & 22.9 & 23.0 \\
\hline \multirow[t]{2}{*}{$\mathrm{CH}_{4}$} & $\mathrm{C}$ & 201.3 & 198.9 & 198.4 & 199.3 & 199.3 & 199.6 & 198.9 & 198.7 \\
\hline & $\mathrm{H}$ & 31.5 & 31.3 & 31.6 & 31.6 & 31.6 & 31.6 & 31.6 & 31.6 \\
\hline \multirow[t]{2}{*}{$\mathrm{C} 2 \mathrm{H} 4$} & $\mathrm{C}$ & 77.3 & 69.7 & 73.3 & 83.0 & 80.7 & 80.8 & 77.6 & 79.3 \\
\hline & $\mathrm{H}$ & 26.3 & 26.0 & 26.5 & 26.7 & 26.7 & 26.7 & 26.6 & 26.7 \\
\hline \multirow[t]{2}{*}{$\mathrm{AlF}$} & $\mathrm{Al}$ & 576.5 & 572.9 & 580.9 & 586.1 & 583.4 & 583.3 & 580.7 & 581.7 \\
\hline & $\mathrm{F}$ & 200.2 & 211.8 & 217.9 & 217.6 & 215.6 & 216.3 & 216.0 & 214.9 \\
\hline $\mathrm{CH}_{3} \mathrm{~F}$ & $\mathrm{C}$ & 127.4 & 122.1 & 127.3 & 130.0 & 129.0 & 129.5 & 128.1 & 128.3 \\
\hline & $\mathrm{F}$ & 479.3 & 482.9 & 477.4 & 472.0 & 472.5 & 473.3 & 474.5 & 473.4 \\
\hline & $\mathrm{H}$ & 27.7 & 27.3 & 28.0 & 28.0 & 28.0 & 28.0 & 27.9 & 27.9 \\
\hline $\mathrm{C}_{3} \mathrm{H}_{4}$ & $\mathrm{C}$ & 194.9 & 192.1 & 193.2 & 194.3 & 193.6 & 194.4 & 193.5 & 193.3 \\
\hline & $\mathrm{C}$ & 90.3 & 83.7 & 87.0 & 96.4 & 94.1 & 94.2 & 90.8 & 92.5 \\
\hline & $\mathrm{H}$ & 24.6 & 24.4 & 24.8 & 25.2 & 25.1 & 25.1 & 25.0 & 25.1 \\
\hline & $\mathrm{H}$ & 30.9 & 30.6 & 31.1 & 31.1 & 31.1 & 31.1 & 31.1 & 31.1 \\
\hline $\mathrm{FCCH}$ & $\mathrm{C}$ & 183.1 & 179.9 & 180.7 & 183.8 & 182.6 & 183.7 & 182.7 & 183.0 \\
\hline & $\mathrm{C}$ & 105.3 & 100.1 & 104.8 & 108.4 & 107.3 & 107.9 & 106.4 & 107.0 \\
\hline & $\mathrm{H}$ & 30.7 & 30.5 & 30.9 & 31.0 & 31.0 & 31.0 & 30.9 & 31.0 \\
\hline & $\mathrm{F}$ & 421.2 & 423.5 & 423.2 & 421.4 & 421.3 & 421.8 & 422.5 & 422.3 \\
\hline $\mathrm{FCN}$ & $\mathrm{F}$ & 373.2 & 374.1 & 377.9 & 376.7 & 376.8 & 376.4 & 376.7 & 376.7 \\
\hline & $\mathrm{C}$ & 88.2 & 82.2 & 85.4 & 92.4 & 90.8 & 91.3 & 88.8 & 90.3 \\
\hline & $\mathrm{N}$ & 122.4 & 117.9 & 116.7 & 131.5 & 128.1 & 129.6 & 125.1 & 127.6 \\
\hline $\mathrm{H}_{2} \mathrm{~S}$ & $\mathrm{~S}$ & 758.5 & 739.0 & 744.8 & 753.3 & 752.2 & 752.1 & 747.7 & 748.9 \\
\hline & $\mathrm{H}$ & 30.9 & 30.5 & 31.1 & 31.3 & 31.3 & 31.2 & 31.2 & 31.2 \\
\hline $\mathrm{HCP}$ & $\mathrm{H}$ & 29.7 & 29.6 & 30.0 & 29.9 & 30.0 & 29.9 & 30.0 & 30.0 \\
\hline & $\mathrm{C}$ & 45.4 & 37.6 & 38.2 & 54.1 & 50.9 & 50.5 & 45.7 & 48.6 \\
\hline & $\mathrm{P}$ & 412.9 & 388.0 & 394.9 & 446.8 & 435.9 & 434.9 & 416.8 & 428.4 \\
\hline $\mathrm{HFCO}$ & $\mathrm{O}$ & -88.0 & -94.3 & -82.9 & -54.6 & -59.0 & -58.7 & -65.9 & -61.9 \\
\hline & $\mathrm{C}$ & 47.0 & 39.6 & 45.0 & 53.3 & 51.3 & 51.7 & 48.8 & 50.6 \\
\hline & $\mathrm{F}$ & 167.2 & 165.3 & 184.0 & 184.5 & 180.3 & 183.2 & 181.6 & 181.9 \\
\hline & $\mathrm{H}$ & 24.3 & 23.9 & 24.6 & 24.6 & 24.6 & 24.6 & 24.6 & 24.6 \\
\hline $\mathrm{H}_{2} \mathrm{C}_{2} \mathrm{O}$ & $\mathrm{C}$ & 196.3 & 193.3 & 194.1 & 195.4 & 195.3 & 195.6 & 194.9 & 194.9 \\
\hline & $\mathrm{C}$ & 3.4 & -6.3 & 1.8 & 11.8 & 10.3 & 9.9 & 6.6 & 8.7 \\
\hline & $\mathrm{O}$ & -3.9 & -5.9 & 4.1 & 18.2 & 17.4 & 16.7 & 13.5 & 14.9 \\
\hline & $\mathrm{H}$ & 29.5 & 29.2 & 29.7 & 29.9 & 29.9 & 29.8 & 29.8 & 29.8 \\
\hline $\mathrm{LiF}$ & $\mathrm{Li}$ & 90.6 & 89.3 & 90.9 & 90.9 & 90.5 & 90.8 & 90.8 & 90.7 \\
\hline & $\mathrm{F}$ & 362.8 & 382.5 & 371.1 & 369.7 & 362.9 & 371.0 & 370.6 & 367.9 \\
\hline $\mathrm{LiH}$ & $\mathrm{H}$ & 26.5 & 26.6 & 26.8 & 26.5 & 26.5 & 26.6 & 26.8 & 26.6 \\
\hline & $\mathrm{Li}$ & 90.0 & 89.3 & 89.9 & 90.9 & 90.6 & 90.5 & 90.2 & 90.3 \\
\hline $\mathrm{N}_{2} \mathrm{O}$ & $\mathrm{N}$ & 114.3 & 106.4 & 107.8 & 125.4 & 122.9 & 121.8 & 116.2 & 120.0 \\
\hline & $\mathrm{N}$ & 22.1 & 12.6 & 13.9 & 34.5 & 32.3 & 29.8 & 23.3 & 28.1 \\
\hline & $\mathrm{O}$ & 197.0 & 199.0 & 197.7 & 207.2 & 206.4 & 205.3 & 202.6 & 204.5 \\
\hline OCS & $\mathrm{O}$ & 98.7 & 96.8 & 100.6 & 112.6 & 111.3 & 110.1 & 106.6 & 108.9 \\
\hline & $\mathrm{C}$ & 37.9 & 30.2 & 32.2 & 47.0 & 45.2 & 43.9 & 39.3 & 42.6 \\
\hline & $\mathrm{S}$ & 810.9 & 796.7 & 811.1 & 823.4 & 820.4 & 822.1 & 816.6 & 818.7 \\
\hline $\mathrm{OF}_{2}$ & $\mathrm{O}$ & -402.8 & -447.1 & -377.4 & -230.0 & -300.5 & -309.3 & -356.8 & -320.7 \\
\hline & $\mathrm{F}$ & 0.0 & -24.0 & 27.2 & 49.3 & 42.4 & 38.6 & 31.1 & 36.0 \\
\hline $\mathrm{H}_{4} \mathrm{C}_{2} \mathrm{O}$ & $\mathrm{O}$ & 362.1 & 363.2 & 364.7 & 359.0 & 357.7 & 361.5 & 362.9 & 360.7 \\
\hline & $\mathrm{C}$ & 158.1 & 153.2 & 157.5 & 159.2 & 158.3 & 159.2 & 158.0 & 158.0 \\
\hline & $\mathrm{H}$ & 29.4 & 29.1 & 29.7 & 29.7 & 29.6 & 29.6 & 29.7 & 29.6 \\
\hline $\mathrm{PN}$ & $\mathrm{N}$ & -336.0 & -344.0 & -348.5 & -268.5 & -286.4 & -293.1 & -317.7 & -301.4 \\
\hline & $\mathrm{P}$ & 89.4 & 50.6 & 58.4 & 177.5 & 152.2 & 137.8 & 100.8 & 129.9 \\
\hline
\end{tabular}


Table 5: Absolute isotropic NMR shieldings in a cc-pVTZ basis. All values are given in ppm. "Extrap." stands for basis-set extrapolated $\operatorname{CCSD}(\mathrm{T})$-shieldings in an aug-cc-pCV[TQ]Z basis set. The data for "Extrap." was taken from Ref. 3, CCSD(T) calculations were done with the CFOUR program 1, 2, MP2 calculations were done with the Turbomole program [4 [6].

\begin{tabular}{|c|c|c|c|c|c|c|c|}
\hline Mol. & Nuc. & $\operatorname{CCSD}(\mathrm{T})$ & Extrap. & $\sigma$-P1@PBE & $\sigma-\mathrm{P} 2 @ \mathrm{PBE}$ & $\sigma$-P3@PBE & $\overline{\text { MP2 }}$ \\
\hline \multirow{2}{*}{$\mathrm{HF}$} & $\mathrm{H}$ & 29.5 & 28.8 & 30.0 & 30.0 & 29.9 & 29.2 \\
\hline & $\mathrm{F}$ & 419.8 & 420.3 & 415.7 & 415.7 & 416.1 & 425.2 \\
\hline \multirow[t]{2}{*}{$\mathrm{CO}$} & $\mathrm{C}$ & 11.3 & 2.2 & 18.4 & 18.2 & 18.8 & 16.8 \\
\hline & $\mathrm{O}$ & -49.8 & -55.0 & -36.5 & -36.6 & -34.4 & -43.3 \\
\hline $\mathrm{N}_{2}$ & $\mathrm{~N}$ & -52.5 & -60.4 & -43.0 & -43.5 & -42.2 & -35.8 \\
\hline \multirow[t]{2}{*}{$\mathrm{H}_{2} \mathrm{O}$} & $\mathrm{O}$ & 344.3 & 338.0 & 338.5 & 338.6 & 338.9 & 352.5 \\
\hline & $\mathrm{H}$ & 31.3 & 30.6 & 31.6 & 31.6 & 31.5 & 31.0 \\
\hline \multirow[t]{3}{*}{$\mathrm{HCN}$} & $\mathrm{H}$ & 29.2 & 29.0 & 29.5 & 29.6 & 29.6 & 29.2 \\
\hline & $\mathrm{C}$ & 91.5 & 84.6 & 94.8 & 94.5 & 95.1 & 93.0 \\
\hline & $\mathrm{N}$ & -7.4 & -14.1 & 3.5 & 3.1 & 3.4 & 5.2 \\
\hline \multirow{3}{*}{$\mathrm{HOF}$} & $\mathrm{O}$ & -44.4 & -68.9 & -19.6 & -19.4 & -14.0 & -28.6 \\
\hline & $\mathrm{H}$ & 20.7 & 19.6 & 21.8 & 21.8 & 21.9 & 20.8 \\
\hline & $\mathrm{F}$ & 188.3 & 192.2 & 190.4 & 191.7 & 193.3 & 191.4 \\
\hline \multirow[t]{2}{*}{$\mathrm{NH}_{3}$} & $\mathrm{~N}$ & 276.2 & 270.7 & 271.3 & 271.4 & 271.6 & 281.8 \\
\hline & $\mathrm{H}$ & 32.0 & 31.4 & 32.1 & 32.1 & 32.1 & 31.8 \\
\hline \multirow{3}{*}{$\mathrm{CH}_{2} \mathrm{O}$} & $\mathrm{O}$ & -376.5 & -378.6 & -333.6 & -333.2 & -325.9 & -335.0 \\
\hline & $\mathrm{C}$ & 13.2 & 1.5 & 24.1 & 24.0 & 24.4 & 13.5 \\
\hline & $\mathrm{H}$ & 22.3 & 22.0 & 23.0 & 23.0 & 23.0 & 22.3 \\
\hline \multirow[t]{2}{*}{$\mathrm{CH}_{4}$} & $\mathrm{C}$ & 201.3 & 198.9 & 198.4 & 198.4 & 198.6 & 203.0 \\
\hline & $\mathrm{H}$ & 31.5 & 31.3 & 31.6 & 31.6 & 31.6 & 31.4 \\
\hline \multirow[t]{2}{*}{$\mathrm{C} 2 \mathrm{H} 4$} & $\mathrm{C}$ & 77.3 & 69.7 & 80.8 & 80.9 & 80.9 & 75.9 \\
\hline & $\mathrm{H}$ & 26.3 & 26.0 & 26.7 & 26.7 & 26.7 & 26.2 \\
\hline \multirow[t]{2}{*}{$\mathrm{AlF}$} & $\mathrm{Al}$ & 576.5 & 572.9 & 583.0 & 582.9 & 583.4 & 575.8 \\
\hline & $\mathrm{F}$ & 200.2 & 211.8 & 214.0 & 213.7 & 214.7 & 197.8 \\
\hline \multirow{3}{*}{$\mathrm{CH}_{3} \mathrm{~F}$} & $\mathrm{C}$ & 127.4 & 122.1 & 128.8 & 128.8 & 129.0 & 126.0 \\
\hline & $\mathrm{F}$ & 479.3 & 482.9 & 475.4 & 475.4 & 475.4 & 485.8 \\
\hline & $\mathrm{H}$ & 27.7 & 27.3 & 28.1 & 28.1 & 28.1 & 27.6 \\
\hline $\mathrm{C}_{3} \mathrm{H}_{4}$ & $\mathrm{C}$ & 194.9 & 192.1 & 194.3 & 194.3 & 194.4 & 195.8 \\
\hline & $\mathrm{C}$ & 90.3 & 83.7 & 94.1 & 94.0 & 93.9 & 91.6 \\
\hline & $\mathrm{H}$ & 24.6 & 24.4 & 25.1 & 25.1 & 25.1 & 24.6 \\
\hline & $\mathrm{H}$ & 30.9 & 30.6 & 31.2 & 31.2 & 31.2 & 30.9 \\
\hline $\mathrm{FCCH}$ & $\mathrm{C}$ & 183.1 & 179.9 & 182.5 & 182.6 & 182.5 & 185.0 \\
\hline & $\mathrm{C}$ & 105.3 & 100.1 & 107.6 & 107.6 & 107.6 & 102.8 \\
\hline & $\mathrm{H}$ & 30.7 & 30.5 & 31.0 & 31.0 & 31.0 & 30.7 \\
\hline & $\mathrm{F}$ & 421.2 & 423.5 & 421.7 & 421.7 & 421.8 & 427.5 \\
\hline $\mathrm{FCN}$ & $\mathrm{F}$ & 373.2 & 374.1 & 373.6 & 373.7 & 374.0 & 382.0 \\
\hline & $\mathrm{C}$ & 88.2 & 82.2 & 90.5 & 90.3 & 90.3 & 87.2 \\
\hline & $\mathrm{N}$ & 122.4 & 117.9 & 125.6 & 125.4 & 125.6 & 135.3 \\
\hline $\mathrm{H}_{2} \mathrm{~S}$ & $\mathrm{~S}$ & 758.5 & 739.0 & 739.5 & 739.6 & 739.8 & 774.0 \\
\hline & $\mathrm{H}$ & 30.9 & 30.5 & 31.4 & 31.4 & 31.3 & 30.7 \\
\hline $\mathrm{HCP}$ & $\mathrm{H}$ & 29.7 & 29.6 & 30.1 & 30.1 & 30.1 & 29.6 \\
\hline & $\mathrm{C}$ & 45.4 & 37.6 & 52.5 & 51.6 & 52.2 & 50.2 \\
\hline & $\mathrm{P}$ & 412.9 & 388.0 & 436.5 & 434.5 & 435.3 & 419.3 \\
\hline $\mathrm{HFCO}$ & $\mathrm{O}$ & -88.0 & -94.3 & -76.7 & -76.3 & -73.4 & -57.8 \\
\hline & $\mathrm{C}$ & 47.0 & 39.6 & 49.9 & 49.9 & 50.2 & 47.4 \\
\hline & $\mathrm{F}$ & 167.2 & 165.3 & 174.4 & 174.2 & 175.5 & 167.4 \\
\hline & $\mathrm{H}$ & 24.3 & 23.9 & 24.6 & 24.6 & 24.6 & 24.2 \\
\hline $\mathrm{H}_{2} \mathrm{C}_{2} \mathrm{O}$ & $\mathrm{C}$ & 196.3 & 193.3 & 194.0 & 194.1 & 194.0 & 201.1 \\
\hline & $\mathrm{C}$ & 3.4 & -6.3 & 5.3 & 5.3 & 5.5 & 7.5 \\
\hline & $\mathrm{O}$ & -3.9 & -5.9 & 3.1 & 2.9 & 5.0 & 29.7 \\
\hline & $\mathrm{H}$ & 29.5 & 29.2 & 29.8 & 29.8 & 29.8 & 29.6 \\
\hline $\mathrm{LiF}$ & $\mathrm{Li}$ & 90.6 & 89.3 & 90.8 & 90.8 & 90.8 & 90.5 \\
\hline & $\mathrm{F}$ & 362.8 & 382.5 & 369.7 & 369.4 & 369.6 & 360.2 \\
\hline $\mathrm{LiH}$ & $\mathrm{H}$ & 26.5 & 26.6 & 26.7 & 26.7 & 26.7 & 26.6 \\
\hline & $\mathrm{Li}$ & 90.0 & 89.3 & 90.6 & 90.6 & 90.6 & 90.0 \\
\hline $\mathrm{N}_{2} \mathrm{O}$ & $\mathrm{N}$ & 114.3 & 106.4 & 118.0 & 117.4 & 118.2 & 137.1 \\
\hline & $\mathrm{N}$ & 22.1 & 12.6 & 29.7 & 28.7 & 29.5 & 40.0 \\
\hline & $\mathrm{O}$ & 197.0 & 199.0 & 200.0 & 200.1 & 201.1 & 214.0 \\
\hline OCS & $\mathrm{O}$ & 98.7 & 96.8 & 108.6 & 108.8 & 109.3 & 111.7 \\
\hline & $\mathrm{C}$ & 37.9 & 30.2 & 44.2 & 43.9 & 44.1 & 45.0 \\
\hline & $\mathrm{S}$ & 810.9 & 796.7 & 811.8 & 812.1 & 812.3 & 830.2 \\
\hline $\mathrm{OF}_{2}$ & $\mathrm{O}$ & -402.8 & -447.1 & -341.9 & -341.7 & -328.0 & -422.1 \\
\hline & $\mathrm{F}$ & 0.0 & -24.0 & 12.4 & 14.1 & 16.7 & 9.6 \\
\hline $\mathrm{H}_{4} \mathrm{C}_{2} \mathrm{O}$ & $\mathrm{O}$ & 362.1 & 363.2 & 359.3 & 359.3 & 359.5 & 370.5 \\
\hline & $\mathrm{C}$ & 158.1 & 153.2 & 158.3 & 158.3 & 158.4 & 157.3 \\
\hline & $\mathrm{H}$ & 29.4 & 29.1 & 29.7 & 29.7 & 29.7 & 29.3 \\
\hline $\mathrm{PN}$ & $\mathrm{N}$ & -336.0 & -344.0 & -299.1 & -303.1 & -301.3 & -248.2 \\
\hline & $\mathrm{P}$ & 89.4 & 50.6 & 132.7 & 126.5 & 128.9 & 152.2 \\
\hline
\end{tabular}


Table 6: Statistics for absolute isotropic NMR shieldings in a cc-pVTZ basis. CCSD(T)/aug-cc$\mathrm{pCV}[\mathrm{TQ}] \mathrm{Z}$ shieldings are used as a reference. $\mathrm{MSD}=$ mean signed deviation; $\mathrm{MAD}=$ mean absolute deviation; $\mathrm{MRD}=$ mean relative deviation [\%]; $\mathrm{MARD}=$ mean absolute relative deviation [\%]; $\mathrm{RMSD}=$ root mean squared deviation.

\begin{tabular}{lrrrrr}
\hline \hline & MSD & MAD & MRD & MARD & RMSD \\
\hline RHF & -9.4 & 17.4 & -40.5 & 51.9 & 34.9 \\
PBE & -18.4 & 18.6 & -41.3 & 42.0 & 31.7 \\
KT2 & -5.7 & 9.3 & 16.5 & 24.4 & 17.7 \\
B3LYP & -19.1 & 19.3 & -52.4 & 53.0 & 30.6 \\
B97-2 & -13.4 & 13.6 & -29.6 & 30.5 & 22.7 \\
CCSD & 4.2 & 5.4 & 19.8 & 21.4 & 11.3 \\
CCSD(T) & 5.0 & 6.3 & 28.2 & 28.7 & 10.6 \\
RPA@HF & 5.3 & 6.1 & 24.2 & 24.4 & 12.8 \\
RPA@BH\&H & 10.0 & 10.7 & 46.4 & 46.5 & 19.4 \\
RPA@B3LYP & 14.4 & 15.2 & 64.5 & 64.7 & 28.4 \\
RPA@KT2 & 15.0 & 16.1 & 68.1 & 68.4 & 30.5 \\
RPA@B97-2 & 12.9 & 13.8 & 59.0 & 59.2 & 25.9 \\
RPA@PBE & 18.9 & 19.9 & 82.0 & 82.3 & 40.4 \\
$\sigma$-P1@PBE & 10.5 & 11.5 & 53.5 & 53.7 & 21.9 \\
$\sigma$-P2@PBE & 10.3 & 11.3 & 52.9 & 53.1 & 21.4 \\
$\sigma$-P3@PBE & 11.2 & 12.1 & 55.1 & 55.3 & 23.3 \\
MP2 & 12.3 & 13.4 & 50.8 & 51.1 & 23.3 \\
\hline \hline
\end{tabular}

Table 7: Statistics for absolute isotropic NMR shieldings in a cc-pVTZ basis. CCSD (T) shieldings are used as a reference. $\mathrm{MSD}=$ mean signed deviation; $\mathrm{MAD}=$ mean absolute deviation; $\mathrm{MRD}=$ mean relative deviation [\%]; MARD=mean absolute relative deviation [\%]; RMSD=root mean squared deviation.

\begin{tabular}{lrrrrr}
\hline \hline & MSD & MAD & MRD & MARD & RMSD \\
\hline RHF & -14.4 & 21.0 & -45.0 & 47.8 & 40.0 \\
PBE & -23.4 & 23.5 & -36.3 & 36.5 & 38.6 \\
KT2 & -10.6 & 11.5 & -3.4 & 11.9 & 21.1 \\
B3LYP & -24.1 & 24.2 & -47.1 & 47.3 & 38.2 \\
B97-2 & -18.4 & 18.4 & -31.7 & 31.8 & 30.2 \\
CCSD & -0.8 & 3.4 & -5.7 & 6.7 & 6.4 \\
CCSD(T) & 0.0 & 0.0 & 0.0 & 0.0 & 0.0 \\
RPA@HF & 0.3 & 5.1 & -0.5 & 9.1 & 9.0 \\
RPA@BH\&H & 5.0 & 5.9 & 13.5 & 13.7 & 12.0 \\
RPA@B3LYP & 9.4 & 10.1 & 23.1 & 23.3 & 20.1 \\
RPA@KT2 & 10.0 & 10.8 & 24.7 & 25.0 & 22.0 \\
RPA@B97-2 & 7.9 & 8.8 & 19.8 & 20.0 & 17.6 \\
RPA@PBE & 13.9 & 14.7 & 31.1 & 31.3 & 31.9 \\
$\sigma$-P1@PBE & 5.5 & 6.9 & 12.8 & 13.2 & 13.3 \\
$\sigma$-P2@PBE & 5.3 & 6.7 & 12.4 & 12.8 & 12.8 \\
$\sigma$-P3@PBE & 6.2 & 7.5 & 14.1 & 14.4 & 14.7 \\
MP2 & 7.3 & 8.3 & 23.5 & 24.1 & 16.8 \\
\hline \hline
\end{tabular}


Table 8: Diamagnetic (-dia) contributions to the absolute isotropic NMR shieldings in a cc-pVTZ basis set. All values are given in ppm. The diamagnetic and paramagnetic contributions to the $\operatorname{CCSD}(\mathrm{T})$ shieldings were computed with the CFOUR 1, 2] program. The gauge-origin is located at the position of the nucleus, for which the shielding is computed.

\begin{tabular}{|c|c|c|c|c|c|c|c|}
\hline Mol. & Nuc. & CCSD(T)-dia & RPA@HF-dia & RPA@PBE-dia & RPA@BH\&H-dia & RPA@B3LYP-dia & RPA@KT2-dia \\
\hline \multirow[t]{2}{*}{$\mathrm{HF}$} & $\mathrm{H}$ & 29.0 & 29.0 & 29.2 & 29.2 & 29.2 & 29.2 \\
\hline & $\mathrm{F}$ & 466.9 & 466.7 & 466.9 & 466.8 & 466.9 & 466.8 \\
\hline \multirow[t]{2}{*}{$\mathrm{CO}$} & $\mathrm{C}$ & 259.7 & 259.9 & 260.2 & 259.9 & 260.0 & 260.2 \\
\hline & $\mathrm{O}$ & 395.7 & 395.3 & 395.0 & 395.3 & 395.2 & 395.1 \\
\hline $\mathrm{N}_{2}$ & $\mathrm{~N}$ & 324.9 & 324.7 & 324.6 & 324.7 & 324.7 & 324.7 \\
\hline \multirow[t]{2}{*}{$\mathrm{H}_{2} \mathrm{O}$} & $\mathrm{O}$ & 383.2 & 382.5 & 383.3 & 382.7 & 383.0 & 383.1 \\
\hline & $\mathrm{H}$ & 30.2 & 30.6 & 30.5 & 30.6 & 30.5 & 30.5 \\
\hline \multirow[t]{3}{*}{$\mathrm{HCN}$} & $\mathrm{H}$ & 28.9 & 29.0 & 29.0 & 29.0 & 29.0 & 28.9 \\
\hline & $\mathrm{C}$ & 259.1 & 259.3 & 259.6 & 259.4 & 259.5 & 259.6 \\
\hline & $\mathrm{N}$ & 334.2 & 332.8 & 332.5 & 332.9 & 332.7 & 332.6 \\
\hline \multirow[t]{3}{*}{$\mathrm{HOF}$} & $\mathrm{O}$ & 379.9 & 379.7 & 381.1 & 380.2 & 380.6 & 381.0 \\
\hline & $\mathrm{H}$ & 28.6 & 28.0 & 28.0 & 28.0 & 28.0 & 28.0 \\
\hline & $\mathrm{F}$ & 466.6 & 466.3 & 467.1 & 466.6 & 466.8 & 467.1 \\
\hline \multirow[t]{2}{*}{$\mathrm{NH}_{3}$} & $\mathrm{~N}$ & 310.5 & 309.3 & 310.6 & 309.8 & 310.2 & 310.3 \\
\hline & $\mathrm{H}$ & 30.3 & 30.9 & 30.7 & 30.8 & 30.7 & 30.6 \\
\hline \multirow[t]{3}{*}{$\mathrm{CH}_{2} \mathrm{O}$} & $\mathrm{O}$ & 398.6 & 399.6 & 399.4 & 399.7 & 399.6 & 399.5 \\
\hline & $\mathrm{C}$ & 246.4 & 245.8 & 247.2 & 246.4 & 246.9 & 247.2 \\
\hline & $\mathrm{H}$ & 28.4 & 29.6 & 29.2 & 29.4 & 29.3 & 29.2 \\
\hline \multirow[t]{2}{*}{$\mathrm{CH}_{4}$} & $\mathrm{C}$ & 247.6 & 246.6 & 248.2 & 247.2 & 247.8 & 247.9 \\
\hline & $\mathrm{H}$ & 29.4 & 29.9 & 29.7 & 29.8 & 29.8 & 29.7 \\
\hline \multirow[t]{2}{*}{$\mathrm{C} 2 \mathrm{H} 4$} & $\mathrm{C}$ & 253.3 & 253.1 & 253.9 & 253.4 & 253.7 & 253.8 \\
\hline & $\mathrm{H}$ & 30.1 & 30.8 & 30.5 & 30.7 & 30.6 & 30.5 \\
\hline \multirow[t]{2}{*}{$\mathrm{AlF}$} & $\mathrm{Al}$ & 791.1 & 790.8 & 791.0 & 790.9 & 790.9 & 791.0 \\
\hline & $\mathrm{F}$ & 467.0 & 466.8 & 466.4 & 466.7 & 466.5 & 466.3 \\
\hline \multirow[t]{3}{*}{$\mathrm{CH}_{3} \mathrm{~F}$} & $\mathrm{C}$ & 238.4 & 240.3 & 242.2 & 241.1 & 241.8 & 241.9 \\
\hline & $\mathrm{F}$ & 469.9 & 471.4 & 471.2 & 471.4 & 471.3 & 471.3 \\
\hline & $\mathrm{H}$ & 28.3 & 29.1 & 28.9 & 29.0 & 28.9 & 28.8 \\
\hline $\mathrm{C}_{3} \mathrm{H}_{4}$ & $\mathrm{C}$ & 246.5 & 236.1 & 238.0 & 236.9 & 237.5 & 237.7 \\
\hline & $\mathrm{C}$ & 254.9 & 245.8 & 247.0 & 246.2 & 246.7 & 246.9 \\
\hline & $\mathrm{H}$ & 29.2 & 25.6 & 25.4 & 25.6 & 25.5 & 25.5 \\
\hline & $\mathrm{H}$ & 29.5 & 22.0 & 21.7 & 21.9 & 21.8 & 21.7 \\
\hline $\mathrm{FCCH}$ & $\mathrm{C}$ & 261.1 & 256.4 & 256.6 & 256.5 & 256.6 & 256.4 \\
\hline & $\mathrm{C}$ & 258.8 & 257.8 & 257.9 & 257.9 & 258.0 & 257.8 \\
\hline & $\mathrm{H}$ & 29.4 & 30.3 & 30.3 & 30.3 & 30.3 & 30.3 \\
\hline & $\mathrm{F}$ & 467.1 & 462.8 & 463.5 & 463.1 & 463.3 & 463.5 \\
\hline $\mathrm{FCN}$ & $\mathrm{F}$ & 465.4 & 464.5 & 465.2 & 464.8 & 465.0 & 465.1 \\
\hline & $\mathrm{C}$ & 263.3 & 263.1 & 263.5 & 263.3 & 263.5 & 263.5 \\
\hline & $\mathrm{N}$ & 330.4 & 329.6 & 329.1 & 329.6 & 329.4 & 329.1 \\
\hline $\mathrm{H}_{2} \mathrm{~S}$ & $\mathrm{~S}$ & 1054.7 & 1054.5 & 1054.1 & 1054.4 & 1054.3 & 1054.2 \\
\hline & $\mathrm{H}$ & 29.5 & 30.2 & 30.0 & 30.1 & 30.1 & 30.0 \\
\hline $\mathrm{HCP}$ & $\mathrm{H}$ & 28.8 & 29.2 & 29.0 & 29.1 & 29.0 & 29.0 \\
\hline & $\mathrm{C}$ & 261.6 & 260.2 & 260.6 & 260.4 & 260.4 & 260.4 \\
\hline & $\mathrm{P}$ & 966.9 & 970.5 & 969.2 & 970.1 & 969.7 & 969.3 \\
\hline $\mathrm{HFCO}$ & $\mathrm{O}$ & 398.4 & 399.6 & 399.6 & 399.8 & 399.7 & 399.6 \\
\hline & $\mathrm{C}$ & 247.5 & 248.3 & 249.7 & 249.0 & 249.4 & 249.7 \\
\hline & $\mathrm{F}$ & 471.1 & 472.0 & 472.2 & 472.1 & 472.2 & 472.2 \\
\hline & $\mathrm{H}$ & 28.1 & 29.9 & 29.6 & 29.8 & 29.7 & 29.6 \\
\hline $\mathrm{H}_{2} \mathrm{C}_{2} \mathrm{O}$ & $\mathrm{C}$ & 257.5 & 256.5 & 257.2 & 256.9 & 257.1 & 257.1 \\
\hline & $\mathrm{C}$ & 256.4 & 255.8 & 256.3 & 256.0 & 256.2 & 256.3 \\
\hline & $\mathrm{O}$ & 397.9 & 396.2 & 396.2 & 396.3 & 396.3 & 396.2 \\
\hline & $\mathrm{H}$ & 29.9 & 30.2 & 29.9 & 30.1 & 30.0 & 29.9 \\
\hline $\mathrm{LiF}$ & $\mathrm{Li}$ & 100.8 & 100.7 & 101.1 & 100.9 & 101.0 & 101.1 \\
\hline & $\mathrm{F}$ & 471.5 & 468.5 & 469.0 & 468.6 & 468.8 & 468.7 \\
\hline $\mathrm{LiH}$ & $\mathrm{H}$ & 26.0 & 26.5 & 26.0 & 26.3 & 26.2 & 26.0 \\
\hline & $\mathrm{Li}$ & 101.7 & 101.2 & 101.3 & 101.2 & 101.2 & 101.3 \\
\hline $\mathrm{N}_{2} \mathrm{O}$ & $\mathrm{N}$ & 326.1 & 324.2 & 324.4 & 324.5 & 324.5 & 324.4 \\
\hline & $\mathrm{N}$ & 320.7 & 319.9 & 320.4 & 320.1 & 320.3 & 320.5 \\
\hline & $\mathrm{O}$ & 398.4 & 397.5 & 397.5 & 397.6 & 397.6 & 397.6 \\
\hline OCS & $\mathrm{O}$ & 394.8 & 393.2 & 393.3 & 393.4 & 393.4 & 393.3 \\
\hline & $\mathrm{C}$ & 260.7 & 258.5 & 259.2 & 258.8 & 259.0 & 259.1 \\
\hline & $\mathrm{S}$ & 1055.5 & 1057.5 & 1056.5 & 1057.2 & 1056.8 & 1056.6 \\
\hline $\mathrm{OF}_{2}$ & $\mathrm{O}$ & 378.5 & 378.6 & 380.6 & 379.4 & 380.0 & 380.6 \\
\hline & $\mathrm{F}$ & 466.5 & 465.8 & 466.6 & 466.2 & 466.4 & 466.6 \\
\hline $\mathrm{H}_{4} \mathrm{C}_{2} \mathrm{O}$ & $\mathrm{O}$ & 391.4 & 388.7 & 389.1 & 389.0 & 389.0 & 389.4 \\
\hline & $\mathrm{C}$ & 246.9 & 245.1 & 246.6 & 245.8 & 246.3 & 246.5 \\
\hline & $\mathrm{H}$ & 29.4 & 28.7 & 28.4 & 28.6 & 28.5 & 28.4 \\
\hline PN & $\mathrm{N}$ & 330.4 & 329.5 & 329.0 & 329.5 & 329.3 & 329.0 \\
\hline & $\mathrm{P}$ & 967.2 & 967.3 & 966.8 & 967.2 & 967.0 & 966.9 \\
\hline
\end{tabular}


Table 9: Paramagnetic (-para) contributions to the absolute isotropic NMR shieldings in a cc-pVTZ basis set. All values are given in ppm. The diamagnetic and paramagnetic contributions to the CCSD(T)shieldings were computed with the CFOUR [1, 2] program. The gauge-origin is located at the position of the nucleus, for which the shielding is computed.

\begin{tabular}{|c|c|c|c|c|c|c|c|}
\hline Mol. & Nuc. & CCSD(T)-para & RPA@HF-para & RPA@PBE-para & RPA@BH\&H-para & RPA@B3LYP-para & RPA@KT2-para \\
\hline \multirow{2}{*}{$\mathrm{HF}$} & $\mathrm{H}$ & 0.5 & 0.4 & 0.9 & 0.5 & 0.7 & 0.9 \\
\hline & $\mathrm{F}$ & -47.2 & -47.7 & -49.0 & -48.2 & -48.6 & -48.6 \\
\hline \multirow[t]{2}{*}{$\mathrm{CO}$} & $\mathrm{C}$ & -248.4 & -254.3 & -231.8 & -245.6 & -238.5 & -236.6 \\
\hline & $\mathrm{O}$ & -445.5 & -443.7 & -416.4 & -433.1 & -424.9 & -421.3 \\
\hline $\mathrm{N}_{2}$ & $\mathrm{~N}$ & -377.5 & -382.9 & -353.4 & -372.0 & -362.5 & -359.4 \\
\hline \multirow{2}{*}{$\mathrm{H}_{2} \mathrm{O}$} & $\mathrm{O}$ & -38.9 & -40.8 & -42.2 & -41.2 & -41.5 & -41.5 \\
\hline & $\mathrm{H}$ & 1.0 & 0.7 & 1.2 & 0.9 & 1.1 & 1.2 \\
\hline \multirow[t]{3}{*}{$\mathrm{HCN}$} & $\mathrm{H}$ & 0.4 & 0.5 & 0.5 & 0.5 & 0.5 & 0.6 \\
\hline & $\mathrm{C}$ & -167.6 & -171.8 & -160.9 & -167.2 & -163.8 & -163.2 \\
\hline & $\mathrm{N}$ & -341.6 & -344.6 & -320.0 & -334.2 & -326.2 & -326.0 \\
\hline \multirow[t]{3}{*}{$\mathrm{HOF}$} & $\mathrm{O}$ & -424.4 & -420.3 & -357.0 & -406.9 & -385.8 & -380.6 \\
\hline & $\mathrm{H}$ & -7.9 & -6.7 & -5.1 & -6.5 & -6.0 & -5.7 \\
\hline & $\mathrm{F}$ & -278.3 & -254.0 & -253.4 & -256.7 & -256.6 & -256.6 \\
\hline \multirow[t]{2}{*}{$\mathrm{NH}_{3}$} & $\mathrm{~N}$ & -34.4 & -36.5 & -37.6 & -36.8 & -36.9 & -37.0 \\
\hline & $\mathrm{H}$ & 1.6 & 1.2 & 1.5 & 1.4 & 1.5 & 1.6 \\
\hline \multirow[t]{3}{*}{$\mathrm{CH}_{2} \mathrm{O}$} & $\mathrm{O}$ & -775.0 & -751.2 & -665.8 & -716.1 & -693.1 & -691.8 \\
\hline & $\mathrm{C}$ & -233.2 & -233.5 & -215.6 & -227.8 & -222.1 & -221.8 \\
\hline & $\mathrm{H}$ & -6.1 & -6.7 & -5.9 & -6.5 & -6.3 & -6.1 \\
\hline \multirow[t]{2}{*}{$\mathrm{CH}_{4}$} & $\mathrm{C}$ & -46.2 & -48.2 & -48.9 & -48.3 & -48.2 & -48.6 \\
\hline & $\mathrm{H}$ & 2.0 & 1.7 & 1.9 & 1.8 & 1.8 & 1.9 \\
\hline \multirow[t]{2}{*}{$\mathrm{C} 2 \mathrm{H} 4$} & $\mathrm{C}$ & -176.1 & -179.8 & -170.9 & -175.8 & -172.9 & -173.1 \\
\hline & $\mathrm{H}$ & -3.8 & -4.3 & -3.8 & -4.1 & -3.9 & -3.8 \\
\hline \multirow[t]{2}{*}{$\mathrm{AlF}$} & $\mathrm{Al}$ & -214.6 & -209.9 & -204.9 & -210.2 & -207.6 & -207.6 \\
\hline & $\mathrm{F}$ & -266.8 & -248.9 & -248.8 & -250.7 & -250.2 & -250.7 \\
\hline \multirow{3}{*}{$\mathrm{CH}_{3} \mathrm{~F}$} & $\mathrm{C}$ & -111.0 & -113.0 & -112.2 & -113.0 & -112.3 & -112.9 \\
\hline & $\mathrm{F}$ & 9.4 & 6.0 & 0.8 & 3.1 & 2.0 & 1.2 \\
\hline & $\mathrm{H}$ & -0.6 & -1.1 & -0.9 & -1.1 & -0.9 & -0.8 \\
\hline $\mathrm{C}_{3} \mathrm{H}_{4}$ & $\mathrm{C}$ & -51.6 & -42.9 & -43.7 & -43.4 & -43.1 & -44.1 \\
\hline & $\mathrm{C}$ & -164.6 & -158.8 & -150.6 & -155.4 & -152.5 & -152.8 \\
\hline & $\mathrm{H}$ & -4.6 & -0.8 & -0.2 & -0.6 & -0.4 & -0.4 \\
\hline & $\mathrm{H}$ & 1.4 & 9.1 & 9.4 & 9.2 & 9.3 & 9.4 \\
\hline $\mathrm{FCCH}$ & $\mathrm{C}$ & -77.9 & -75.7 & -72.8 & -73.8 & -72.9 & -73.8 \\
\hline & $\mathrm{C}$ & -153.4 & -153.0 & -149.5 & -151.5 & -150.1 & -150.5 \\
\hline & $\mathrm{H}$ & 1.3 & 0.6 & 0.7 & 0.6 & 0.7 & 0.7 \\
\hline & $\mathrm{F}$ & -45.8 & -39.6 & -42.1 & -40.6 & -41.5 & -42.2 \\
\hline $\mathrm{FCN}$ & $\mathrm{F}$ & -92.3 & -86.6 & -88.5 & -88.1 & -88.6 & -88.3 \\
\hline & $\mathrm{C}$ & -175.1 & -177.7 & -171.1 & -174.5 & -172.2 & -172.7 \\
\hline & $\mathrm{N}$ & -207.9 & -212.9 & -197.6 & -204.5 & -199.8 & -201.0 \\
\hline $\mathrm{H}_{2} \mathrm{~S}$ & $\mathrm{~S}$ & -296.2 & -309.7 & -300.8 & -306.7 & -302.2 & -302.0 \\
\hline & $\mathrm{H}$ & 1.4 & 0.9 & 1.3 & 1.1 & 1.1 & 1.3 \\
\hline $\mathrm{HCP}$ & $\mathrm{H}$ & 0.9 & 0.8 & 0.9 & 0.9 & 0.9 & 1.0 \\
\hline & $\mathrm{C}$ & -216.3 & -222.0 & -206.5 & -214.7 & -209.9 & -209.5 \\
\hline & $\mathrm{P}$ & -554.0 & -575.6 & -522.4 & -553.3 & -534.8 & -533.4 \\
\hline $\mathrm{HFCO}$ & $\mathrm{O}$ & -486.4 & -482.5 & -454.2 & -465.7 & -458.4 & -458.6 \\
\hline & $\mathrm{C}$ & -200.5 & -203.3 & -196.4 & -200.2 & -197.7 & -198.4 \\
\hline & $\mathrm{F}$ & -303.9 & -288.0 & -287.7 & -290.5 & -289.0 & -291.9 \\
\hline & $\mathrm{H}$ & -3.8 & -5.3 & -5.0 & -5.2 & -5.1 & -5.0 \\
\hline $\mathrm{H}_{2} \mathrm{C}_{2} \mathrm{O}$ & $\mathrm{C}$ & -61.3 & -62.4 & -61.8 & -62.0 & -61.5 & -61.8 \\
\hline & $\mathrm{C}$ & -252.9 & -254.0 & -244.5 & -249.4 & -246.3 & -246.0 \\
\hline & $\mathrm{O}$ & -401.8 & -392.1 & -378.0 & -382.8 & -379.6 & -378.8 \\
\hline & $\mathrm{H}$ & -0.3 & -0.5 & 0.0 & -0.3 & -0.2 & 0.0 \\
\hline $\mathrm{LiF}$ & $\mathrm{Li}$ & -10.1 & -9.8 & -10.2 & -10.1 & -10.2 & -10.6 \\
\hline & $\mathrm{F}$ & -101.2 & -97.4 & -99.3 & -98.0 & -97.8 & -105.8 \\
\hline $\mathrm{LiH}$ & $\mathrm{H}$ & 0.5 & 0.3 & 0.5 & 0.5 & 0.4 & 0.5 \\
\hline & $\mathrm{Li}$ & -11.7 & -11.3 & -10.4 & -11.0 & -10.7 & -10.7 \\
\hline $\mathrm{N}_{2} \mathrm{O}$ & $\mathrm{N}$ & -211.8 & -216.4 & -199.0 & -208.3 & -202.7 & -201.5 \\
\hline & $\mathrm{N}$ & -298.6 & -306.0 & -285.9 & -296.8 & -290.5 & -288.2 \\
\hline & $\mathrm{O}$ & -201.4 & -199.8 & -190.3 & -195.0 & -192.3 & -191.2 \\
\hline OCS & $\mathrm{O}$ & -296.1 & -292.6 & -280.7 & -286.8 & -283.3 & -282.0 \\
\hline & $\mathrm{C}$ & -222.9 & -226.3 & -212.2 & -219.5 & -215.1 & -213.9 \\
\hline & $\mathrm{S}$ & -244.6 & -246.4 & -233.1 & -240.6 & -234.7 & -236.2 \\
\hline $\mathrm{OF}_{2}$ & $\mathrm{O}$ & -781.3 & -756.0 & -610.6 & -736.2 & -689.3 & -681.1 \\
\hline & $\mathrm{F}$ & -466.5 & -438.6 & -417.3 & -435.1 & -427.8 & -424.2 \\
\hline $\mathrm{H}_{4} \mathrm{C}_{2} \mathrm{O}$ & $\mathrm{O}$ & -29.3 & -24.0 & -30.1 & -26.1 & -27.5 & -31.7 \\
\hline & $\mathrm{C}$ & -88.8 & -87.6 & -87.4 & -87.8 & -87.1 & -88.2 \\
\hline & $\mathrm{H}$ & 0.0 & 1.0 & 1.3 & 1.1 & 1.1 & 1.2 \\
\hline $\mathrm{PN}$ & $\mathrm{N}$ & -666.4 & -678.0 & -597.5 & -647.2 & -622.4 & -615.4 \\
\hline & $\mathrm{P}$ & -877.8 & -908.9 & -789.3 & -866.4 & -829.2 & -814.7 \\
\hline
\end{tabular}


Table 10: Diamagnetic (-dia) contributions to the absolute isotropic NMR shieldings in a cc-pVTZ basis set. All values are given in ppm. The diamagnetic and paramagnetic contributions to the CCSD(T)shieldings were computed with the CFOUR 1, 2 program. The gauge-origin is located at the the position of the nucleus, for which the shielding is computed.

\begin{tabular}{|c|c|c|c|c|c|c|c|}
\hline Mol. & Nuc. & CCSD(T)-dia & HF(num.)-dia & PBE(num.)-dia & BH\&H(num.)-dia & B3LYP(num.)-dia & KT2(num.)-dia \\
\hline \multirow[t]{2}{*}{$\mathrm{HF}$} & $\mathrm{H}$ & 29.0 & 28.5 & 29.8 & 29.0 & 29.5 & 29.5 \\
\hline & $\mathrm{F}$ & 466.9 & 466.0 & 467.3 & 467.0 & 467.2 & 470.9 \\
\hline \multirow[t]{2}{*}{$\mathrm{CO}$} & $\mathrm{C}$ & 259.7 & 259.4 & 259.6 & 259.4 & 259.3 & 261.2 \\
\hline & $\mathrm{O}$ & 395.7 & 394.1 & 397.7 & 396.1 & 397.2 & 401.3 \\
\hline $\mathrm{N}_{2}$ & $\mathrm{~N}$ & 324.9 & 324.3 & 325.6 & 325.0 & 325.2 & 327.4 \\
\hline \multirow{2}{*}{$\mathrm{H}_{2} \mathrm{O}$} & $\mathrm{O}$ & 383.2 & 381.1 & 382.3 & 382.1 & 382.2 & 386.1 \\
\hline & $\mathrm{H}$ & 30.2 & 30.5 & 31.5 & 30.9 & 31.3 & 31.2 \\
\hline \multirow[t]{3}{*}{$\mathrm{HCN}$} & $\mathrm{H}$ & 28.9 & 29.0 & 29.7 & 29.3 & 29.7 & 29.6 \\
\hline & $\mathrm{C}$ & 259.1 & 259.4 & 258.0 & 258.7 & 257.9 & 259.7 \\
\hline & $\mathrm{N}$ & 334.2 & 330.9 & 336.6 & 333.8 & 335.6 & 338.9 \\
\hline \multirow[t]{3}{*}{$\mathrm{HOF}$} & $\mathrm{O}$ & 379.9 & 377.7 & 379.9 & 379.0 & 379.5 & 383.2 \\
\hline & $\mathrm{H}$ & 28.6 & 27.5 & 28.9 & 28.0 & 28.5 & 28.5 \\
\hline & $\mathrm{F}$ & 466.6 & 466.1 & 466.2 & 466.4 & 466.4 & 469.2 \\
\hline \multirow[t]{2}{*}{$\mathrm{NH}_{3}$} & $\mathrm{~N}$ & 310.5 & 307.2 & 308.9 & 308.3 & 308.4 & 312.5 \\
\hline & $\mathrm{H}$ & 30.3 & 30.9 & 31.5 & 31.1 & 31.3 & 31.1 \\
\hline \multirow[t]{3}{*}{$\mathrm{CH}_{2} \mathrm{O}$} & $\mathrm{O}$ & 398.6 & 398.0 & 402.2 & 400.4 & 401.8 & 405.3 \\
\hline & $\mathrm{C}$ & 246.4 & 243.8 & 245.2 & 244.1 & 244.1 & 247.6 \\
\hline & $\mathrm{H}$ & 28.4 & 29.8 & 29.6 & 29.7 & 29.7 & 29.2 \\
\hline \multirow[t]{2}{*}{$\mathrm{CH}_{4}$} & $\mathrm{C}$ & 247.6 & 243.7 & 248.0 & 245.4 & 246.0 & 251.1 \\
\hline & $\mathrm{H}$ & 29.4 & 30.0 & 30.3 & 30.0 & 30.1 & 29.8 \\
\hline \multirow[t]{2}{*}{$\mathrm{C} 2 \mathrm{H} 4$} & $\mathrm{C}$ & 253.3 & 251.7 & 253.3 & 252.2 & 252.2 & 255.9 \\
\hline & $\mathrm{H}$ & 30.1 & 31.1 & 31.1 & 31.0 & 31.1 & 30.5 \\
\hline \multirow[t]{2}{*}{$\mathrm{AlF}$} & $\mathrm{Al}$ & 791.1 & 790.8 & 792.4 & 791.7 & 792.1 & 794.7 \\
\hline & $\mathrm{F}$ & 467.0 & 466.0 & 467.9 & 467.6 & 468.4 & 471.0 \\
\hline \multirow{3}{*}{$\mathrm{CH}_{3} \mathrm{~F}$} & $\mathrm{C}$ & 238.4 & 237.4 & 240.5 & 238.4 & 238.6 & 243.6 \\
\hline & $\mathrm{F}$ & 469.9 & 470.5 & 473.7 & 472.6 & 473.8 & 476.4 \\
\hline & $\mathrm{H}$ & 28.3 & 29.1 & 29.3 & 29.1 & 29.1 & 28.8 \\
\hline $\mathrm{C}_{3} \mathrm{H}_{4}$ & $\mathrm{C}$ & 246.5 & 233.2 & 236.5 & 234.4 & 235.0 & 238.7 \\
\hline & $\mathrm{C}$ & 254.9 & 244.6 & 243.7 & 244.2 & 243.5 & 245.8 \\
\hline & $\mathrm{H}$ & 29.2 & 25.7 & 26.4 & 26.0 & 26.3 & 25.8 \\
\hline & $\mathrm{H}$ & 29.5 & 22.3 & 21.8 & 21.9 & 21.8 & 20.6 \\
\hline $\mathrm{FCCH}$ & $\mathrm{C}$ & 261.1 & 255.8 & 256.3 & 256.0 & 255.7 & 258.4 \\
\hline & $\mathrm{C}$ & 258.8 & 257.2 & 257.3 & 257.0 & 256.7 & 259.9 \\
\hline & $\mathrm{H}$ & 29.4 & 30.2 & 31.1 & 30.6 & 31.0 & 31.0 \\
\hline & $\mathrm{F}$ & 467.1 & 460.9 & 463.7 & 463.0 & 463.9 & 468.0 \\
\hline $\mathrm{FCN}$ & $\mathrm{F}$ & 465.4 & 462.8 & 466.2 & 465.0 & 466.1 & 470.3 \\
\hline & $\mathrm{C}$ & 263.3 & 262.6 & 262.3 & 262.3 & 262.0 & 264.1 \\
\hline & $\mathrm{N}$ & 330.4 & 328.0 & 333.6 & 330.7 & 332.3 & 335.8 \\
\hline $\mathrm{H}_{2} \mathrm{~S}$ & $\mathrm{~S}$ & 1054.7 & 1055.1 & 1056.7 & 1056.1 & 1056.5 & 1059.5 \\
\hline & $\mathrm{H}$ & 29.5 & 30.2 & 30.1 & 30.1 & 30.2 & 29.9 \\
\hline $\mathrm{HCP}$ & $\mathrm{H}$ & 28.8 & 29.1 & 30.0 & 29.5 & 29.9 & 30.0 \\
\hline & $\mathrm{C}$ & 261.6 & 259.4 & 259.0 & 259.7 & 259.6 & 262.9 \\
\hline & $\mathrm{P}$ & 966.9 & 971.7 & 976.2 & 974.1 & 975.5 & 979.9 \\
\hline $\mathrm{HFCO}$ & $\mathrm{O}$ & 398.4 & 398.0 & 402.7 & 400.5 & 401.9 & 405.9 \\
\hline & $\mathrm{C}$ & 247.5 & 246.4 & 247.9 & 246.7 & 246.8 & 249.9 \\
\hline & $\mathrm{F}$ & 471.1 & 470.7 & 474.4 & 473.2 & 474.4 & 477.5 \\
\hline & $\mathrm{H}$ & 28.1 & 29.9 & 30.6 & 30.1 & 30.4 & 30.0 \\
\hline $\mathrm{H}_{2} \mathrm{C}_{2} \mathrm{O}$ & $\mathrm{C}$ & 257.5 & 254.9 & 258.5 & 256.2 & 256.7 & 260.8 \\
\hline & $\mathrm{C}$ & 256.4 & 255.5 & 254.7 & 255.2 & 254.8 & 256.9 \\
\hline & $\mathrm{O}$ & 397.9 & 394.5 & 398.3 & 396.6 & 397.6 & 401.6 \\
\hline & $\mathrm{H}$ & 29.9 & 30.4 & 30.7 & 30.4 & 30.6 & 30.0 \\
\hline $\mathrm{LiF}$ & $\mathrm{Li}$ & 100.8 & 100.4 & 101.9 & 101.1 & 101.6 & 104.7 \\
\hline & $\mathrm{F}$ & 471.5 & 467.8 & 465.9 & 467.3 & 466.7 & 469.6 \\
\hline $\mathrm{LiH}$ & $\mathrm{H}$ & 26.0 & 26.2 & 25.8 & 26.1 & 26.1 & 25.8 \\
\hline & $\mathrm{Li}$ & 101.7 & 101.4 & 101.9 & 101.8 & 101.8 & 104.9 \\
\hline $\mathrm{N}_{2} \mathrm{O}$ & $\mathrm{N}$ & 326.1 & 322.9 & 326.7 & 324.5 & 325.4 & 328.7 \\
\hline & $\mathrm{N}$ & 320.7 & 319.6 & 318.1 & 319.1 & 318.6 & 321.1 \\
\hline & $\mathrm{O}$ & 398.4 & 396.2 & 399.2 & 397.8 & 398.8 & 401.6 \\
\hline OCS & $\mathrm{O}$ & 394.8 & 391.5 & 395.3 & 393.6 & 394.6 & 398.8 \\
\hline & $\mathrm{C}$ & 260.7 & 257.5 & 257.2 & 257.6 & 257.5 & 260.1 \\
\hline & $\mathrm{S}$ & 1055.5 & 1058.5 & 1060.8 & 1059.7 & 1060.4 & 1064.1 \\
\hline $\mathrm{OF}_{2}$ & $\mathrm{O}$ & 378.5 & 376.2 & 378.9 & 377.5 & 378.2 & 381.8 \\
\hline & $\mathrm{F}$ & 466.5 & 464.8 & 466.5 & 465.8 & 466.3 & 469.5 \\
\hline $\mathrm{H}_{4} \mathrm{C}_{2} \mathrm{O}$ & $\mathrm{O}$ & 391.4 & 387.8 & 389.7 & 389.4 & 390.3 & 391.2 \\
\hline & $\mathrm{C}$ & 246.9 & 242.8 & 245.4 & 243.6 & 243.8 & 247.8 \\
\hline & $\mathrm{H}$ & 29.4 & 28.9 & 29.1 & 28.8 & 28.9 & 28.1 \\
\hline $\mathrm{PN}$ & $\mathrm{N}$ & 330.4 & 327.3 & 331.1 & 329.8 & 331.3 & 334.3 \\
\hline & $\mathrm{P}$ & 967.2 & 967.9 & 969.5 & 968.7 & 969.1 & 971.8 \\
\hline
\end{tabular}


Table 11: Paramagnetic (-para) contributions to the absolute isotropic NMR shieldings in a cc-pVTZ basis set. All values are given in ppm. The diamagnetic and paramagnetic contributions to the $\operatorname{CCSD}(\mathrm{T})-$ shieldings were computed with the CFOUR 1, 2] program. The gauge-origin is located at the the position of the nucleus, for which the shielding is computed.

\begin{tabular}{|c|c|c|c|c|c|c|c|}
\hline Mol. & Nuc. & CCSD(T)-para & $\mathrm{HF}$ (num.)-para & PBE(num.)-para & BH\&H(num.)-para & B3LYP(num.)-para & KT2(num.)-para \\
\hline \multirow{2}{*}{$\mathrm{HF}$} & $\mathrm{H}$ & 0.5 & 0.0 & 0.4 & 0.1 & 0.3 & 0.8 \\
\hline & $\mathrm{F}$ & -47.2 & -50.4 & -54.8 & -52.2 & -54.3 & -59.0 \\
\hline \multirow[t]{2}{*}{$\mathrm{CO}$} & $\mathrm{C}$ & -248.4 & -281.2 & -266.0 & -277.5 & -272.2 & -246.7 \\
\hline & $\mathrm{O}$ & -445.5 & -481.3 & -473.8 & -480.4 & -477.2 & -456.3 \\
\hline $\mathrm{N}_{2}$ & $\mathrm{~N}$ & -377.5 & -433.1 & -402.7 & -421.5 & -411.9 & -380.3 \\
\hline \multirow{2}{*}{$\mathrm{H}_{2} \mathrm{O}$} & $\mathrm{O}$ & -38.9 & -45.7 & -44.9 & -45.6 & -46.4 & -50.4 \\
\hline & $\mathrm{H}$ & 1.0 & 0.4 & 0.2 & 0.3 & 0.2 & 0.6 \\
\hline \multirow[t]{3}{*}{$\mathrm{HCN}$} & $\mathrm{H}$ & 0.4 & 0.3 & -0.4 & 0.1 & -0.3 & -0.6 \\
\hline & $\mathrm{C}$ & -167.6 & -185.0 & -179.8 & -185.1 & -183.0 & -167.1 \\
\hline & $\mathrm{N}$ & -341.6 & -378.2 & -374.2 & -380.9 & -379.6 & -351.5 \\
\hline \multirow[t]{3}{*}{$\mathrm{HOF}$} & $\mathrm{O}$ & -424.4 & -497.0 & -484.5 & -494.9 & -494.0 & -454.1 \\
\hline & $\mathrm{H}$ & -7.9 & -7.9 & -8.8 & -8.1 & -8.5 & -7.8 \\
\hline & $\mathrm{F}$ & -278.3 & -179.6 & -316.7 & -249.4 & -292.8 & -306.3 \\
\hline \multirow[t]{2}{*}{$\mathrm{NH}_{3}$} & $\mathrm{~N}$ & -34.4 & -38.7 & -39.5 & -40.0 & -41.2 & -41.4 \\
\hline & $\mathrm{H}$ & 1.6 & 1.0 & 0.7 & 0.9 & 0.9 & 1.1 \\
\hline \multirow[t]{3}{*}{$\mathrm{CH}_{2} \mathrm{O}$} & $\mathrm{O}$ & -775.0 & -841.6 & -847.6 & -858.2 & -855.1 & -782.5 \\
\hline & $\mathrm{C}$ & -233.2 & -245.9 & -260.8 & -255.8 & -259.6 & -241.9 \\
\hline & $\mathrm{H}$ & -6.1 & -7.1 & -8.6 & -7.7 & -8.1 & -7.7 \\
\hline \multirow[t]{2}{*}{$\mathrm{CH}_{4}$} & $\mathrm{C}$ & -46.2 & -47.0 & -54.4 & -51.5 & -54.5 & -52.6 \\
\hline & $\mathrm{H}$ & 2.0 & 1.6 & 1.2 & 1.6 & 1.5 & 1.8 \\
\hline \multirow[t]{2}{*}{$\mathrm{C} 2 \mathrm{H} 4$} & $\mathrm{C}$ & -176.1 & -187.9 & -196.9 & -195.0 & -197.7 & -184.4 \\
\hline & $\mathrm{H}$ & -3.8 & -4.8 & -5.4 & -4.9 & -5.1 & -4.5 \\
\hline \multirow[t]{2}{*}{$\mathrm{AlF}$} & $\mathrm{Al}$ & -214.6 & -207.7 & -247.8 & -227.6 & -241.3 & -235.3 \\
\hline & $\mathrm{F}$ & -266.8 & -249.7 & -336.5 & -286.5 & -313.9 & -336.2 \\
\hline \multirow{3}{*}{$\mathrm{CH}_{3} \mathrm{~F}$} & $\mathrm{C}$ & -111.0 & -109.6 & -129.2 & -120.4 & -127.1 & -123.9 \\
\hline & $\mathrm{F}$ & 9.4 & 14.4 & -15.9 & 0.8 & -11.0 & -18.1 \\
\hline & $\mathrm{H}$ & -0.6 & -1.1 & -2.1 & -1.4 & -1.6 & -1.5 \\
\hline $\mathrm{C}_{3} \mathrm{H}_{4}$ & C & -51.6 & -38.0 & -56.4 & -47.7 & -54.1 & -52.7 \\
\hline & $\mathrm{C}$ & -164.6 & -169.7 & -173.6 & -174.5 & -176.1 & -162.1 \\
\hline & $\mathrm{H}$ & -4.6 & -1.5 & -2.1 & -1.8 & -1.9 & -1.4 \\
\hline & $\mathrm{H}$ & 1.4 & 8.7 & 8.9 & 9.0 & 9.0 & 10.1 \\
\hline $\mathrm{FCCH}$ & $\mathrm{C}$ & -77.9 & -77.9 & -82.6 & -82.3 & -83.3 & -74.2 \\
\hline & $\mathrm{C}$ & -153.4 & -152.9 & -168.1 & -162.5 & -167.6 & -160.0 \\
\hline & $\mathrm{H}$ & 1.3 & 0.4 & 0.0 & 0.2 & 0.0 & -0.1 \\
\hline & $\mathrm{F}$ & -45.8 & -35.8 & -77.3 & -54.1 & -66.5 & -76.4 \\
\hline $\mathrm{FCN}$ & $\mathrm{F}$ & -92.3 & -87.6 & -128.5 & -104.8 & -116.7 & -133.5 \\
\hline & $\mathrm{C}$ & -175.1 & -183.8 & -186.5 & -188.3 & -189.5 & -175.8 \\
\hline & $\mathrm{N}$ & -207.9 & -234.7 & -229.6 & -238.1 & -236.1 & -212.2 \\
\hline $\mathrm{H}_{2} \mathrm{~S}$ & $\mathrm{~S}$ & -296.2 & -319.9 & -328.9 & -333.0 & -342.5 & -321.5 \\
\hline & $\mathrm{H}$ & 1.4 & 0.6 & 0.9 & 0.8 & 0.9 & 1.1 \\
\hline $\mathrm{HCP}$ & $\mathrm{H}$ & 0.9 & 1.1 & -0.4 & 0.5 & 0.0 & -0.7 \\
\hline & $\mathrm{C}$ & -216.3 & -242.3 & -236.8 & -244.6 & -243.0 & -225.1 \\
\hline & $\mathrm{P}$ & -554.0 & -616.6 & -638.4 & -642.0 & -646.7 & -595.0 \\
\hline HFCO & $\mathrm{O}$ & -486.4 & -524.6 & -522.1 & -534.7 & -531.7 & -496.2 \\
\hline & $\mathrm{C}$ & -200.5 & -209.1 & -218.3 & -216.7 & -219.6 & -206.3 \\
\hline & $\mathrm{F}$ & -303.9 & -282.4 & -381.3 & -326.5 & -357.5 & -368.5 \\
\hline & $\mathrm{H}$ & -3.8 & -5.3 & -7.0 & -5.9 & -6.5 & -6.4 \\
\hline $\mathrm{H}_{2} \mathrm{C}_{2} \mathrm{O}$ & $\mathrm{C}$ & -61.3 & -63.9 & -69.7 & -68.0 & -70.0 & -65.5 \\
\hline & $\mathrm{C}$ & -252.9 & -264.5 & -262.1 & -269.0 & -268.7 & -249.4 \\
\hline & $\mathrm{O}$ & -401.8 & -421.2 & -415.1 & -426.1 & -423.4 & -401.2 \\
\hline & $\mathrm{H}$ & -0.3 & -0.9 & -1.2 & -0.9 & -1.0 & -0.4 \\
\hline $\mathrm{LiF}$ & $\mathrm{Li}$ & -10.1 & -8.6 & -13.6 & -10.9 & -12.5 & -12.5 \\
\hline & $\mathrm{F}$ & -101.2 & -94.9 & -150.1 & -122.7 & -140.8 & -129.6 \\
\hline $\mathrm{LiH}$ & $\mathrm{H}$ & 0.5 & 0.3 & 0.5 & 0.5 & 0.4 & 0.6 \\
\hline & $\mathrm{Li}$ & -11.7 & -11.3 & -12.7 & -12.5 & -12.6 & -11.7 \\
\hline $\mathrm{N}_{2} \mathrm{O}$ & $\mathrm{N}$ & -211.8 & -255.8 & -227.4 & -245.6 & -237.0 & -219.5 \\
\hline & $\mathrm{N}$ & -298.6 & -346.7 & -305.5 & -331.8 & -320.1 & -298.4 \\
\hline & $\mathrm{O}$ & -201.4 & -225.7 & -225.6 & -229.1 & -229.0 & -226.8 \\
\hline OCS & $\mathrm{O}$ & -296.1 & -315.6 & -321.7 & -320.4 & -322.2 & -314.8 \\
\hline & $\mathrm{C}$ & -222.9 & -245.6 & -225.3 & -240.8 & -235.1 & -215.5 \\
\hline & $\mathrm{S}$ & -244.6 & -264.8 & -289.5 & -283.7 & -291.7 & -279.1 \\
\hline $\mathrm{OF}_{2}$ & $\mathrm{O}$ & -781.3 & -796.1 & -965.7 & -869.5 & -927.8 & -883.3 \\
\hline & $\mathrm{F}$ & -466.5 & -424.1 & -530.1 & -479.1 & -513.5 & -511.9 \\
\hline $\mathrm{H}_{4} \mathrm{C}_{2} \mathrm{O}$ & $\mathrm{O}$ & -29.3 & -9.9 & -59.6 & -36.1 & -53.1 & -53.8 \\
\hline & $\mathrm{C}$ & -88.8 & -84.1 & -102.4 & -94.2 & -100.6 & -96.9 \\
\hline & $\mathrm{H}$ & 0.0 & 0.9 & 0.1 & 0.7 & 0.5 & 1.2 \\
\hline $\mathrm{PN}$ & $\mathrm{N}$ & -666.4 & -836.4 & -734.9 & -796.4 & -766.4 & -695.7 \\
\hline & $\mathrm{P}$ & -877.8 & -1059.1 & -995.6 & -1043.9 & -1020.4 & -921.9 \\
\hline
\end{tabular}


Table 12: Testing influence of $\delta$ and DFT grid and gauge-origin independence in a cc-pVTZ basis set. All values are given in ppm. "RPA@HF0.05" denotes calculations with $\delta=0.05$ instead of $\delta=0.1$ as in the other calculations. RPA@HF_trans denotes calculations after translating the molecule by 5.0 Angstroem in $\mathrm{x}-, \mathrm{y}-$ and $\mathrm{z}$-direction, respectively. The gauge-origin is located at $(0.0,0.0,0.0)$. The "RPA@PBE" calculations are performed with a g5 grid; in contrast, a g7 grid is used in the "RPA@PBEg7" calculations.

\begin{tabular}{|c|c|c|c|c|c|c|}
\hline Mol. & Nuc. & RPA@HF & RPA@HF0.05 & RPA@HF_trans & RPA@PBE & RPA@PBE-g7 \\
\hline \multirow[t]{2}{*}{$\mathrm{HF}$} & $\mathrm{H}$ & 29.4 & 29.4 & 29.4 & 30.1 & 30.1 \\
\hline & $\mathrm{F}$ & 419.0 & 419.0 & 419.0 & 417.9 & 417.9 \\
\hline \multirow[t]{2}{*}{$\mathrm{CO}$} & $\mathrm{C}$ & 5.6 & 5.6 & 5.6 & 28.4 & 28.4 \\
\hline & $\mathrm{O}$ & -48.4 & -48.4 & -48.4 & -21.4 & -21.4 \\
\hline $\mathrm{N}_{2}$ & $\mathrm{~N}$ & -58.2 & -58.2 & -58.2 & -28.8 & -28.8 \\
\hline \multirow[t]{2}{*}{$\mathrm{H}_{2} \mathrm{O}$} & $\mathrm{O}$ & 341.7 & 341.7 & 341.7 & 341.1 & 341.1 \\
\hline & $\mathrm{H}$ & 31.3 & 31.3 & 31.3 & 31.7 & 31.7 \\
\hline \multirow{3}{*}{$\mathrm{HCN}$} & $\mathrm{H}$ & 29.5 & 29.5 & 29.5 & 29.5 & 29.5 \\
\hline & $\mathrm{C}$ & 87.5 & 87.5 & 87.5 & 98.7 & 98.7 \\
\hline & $\mathrm{N}$ & -11.8 & -11.8 & -11.8 & 12.5 & 12.5 \\
\hline \multirow[t]{3}{*}{$\mathrm{HOF}$} & $\mathrm{O}$ & -40.6 & -40.7 & -40.6 & 24.1 & 24.1 \\
\hline & $\mathrm{H}$ & 21.3 & 21.3 & 21.3 & 22.9 & 22.9 \\
\hline & $\mathrm{F}$ & 212.3 & 212.2 & 212.3 & 213.7 & 213.7 \\
\hline \multirow[t]{2}{*}{$\mathrm{NH}_{3}$} & $\mathrm{~N}$ & 272.8 & 272.8 & 272.8 & 273.0 & 273.0 \\
\hline & $\mathrm{H}$ & 32.1 & 32.1 & 32.1 & 32.2 & 32.2 \\
\hline \multirow[t]{3}{*}{$\mathrm{CH}_{2} \mathrm{O}$} & $\mathrm{O}$ & -351.6 & -351.8 & -351.6 & -266.4 & -266.4 \\
\hline & $\mathrm{C}$ & 12.3 & 12.3 & 12.3 & 31.6 & 31.6 \\
\hline & $\mathrm{H}$ & 22.9 & 22.9 & 22.9 & 23.3 & 23.3 \\
\hline \multirow[t]{2}{*}{$\mathrm{CH}_{4}$} & $\mathrm{C}$ & 198.4 & 198.4 & 198.4 & 199.3 & 199.3 \\
\hline & $\mathrm{H}$ & 31.6 & 31.6 & 31.6 & 31.6 & 31.6 \\
\hline \multirow[t]{2}{*}{$\mathrm{C} 2 \mathrm{H} 4$} & $\mathrm{C}$ & 73.3 & 73.3 & 73.3 & 83.0 & 83.0 \\
\hline & $\mathrm{H}$ & 26.5 & 26.5 & 26.5 & 26.7 & 26.7 \\
\hline \multirow[t]{2}{*}{$\mathrm{AlF}$} & $\mathrm{Al}$ & 580.9 & 580.9 & 580.9 & 586.1 & 586.1 \\
\hline & $\mathrm{F}$ & 217.9 & 218.0 & 217.9 & 217.6 & 217.6 \\
\hline \multirow{3}{*}{$\mathrm{CH}_{3} \mathrm{~F}$} & $\mathrm{C}$ & 127.3 & 127.3 & 127.3 & 130.0 & 130.0 \\
\hline & $\mathrm{F}$ & 477.4 & 477.4 & 477.4 & 472.0 & 472.0 \\
\hline & $\mathrm{H}$ & 28.0 & 28.0 & 28.0 & 28.0 & 28.0 \\
\hline $\mathrm{C}_{3} \mathrm{H}_{4}$ & $\mathrm{C}$ & 193.2 & 193.2 & 193.2 & 194.3 & 194.3 \\
\hline & $\mathrm{C}$ & 87.0 & 87.0 & 87.0 & 96.4 & 96.4 \\
\hline & $\mathrm{H}$ & 24.8 & 24.8 & 24.8 & 25.2 & 25.2 \\
\hline & $\mathrm{H}$ & 31.1 & 31.1 & 31.1 & 31.1 & 31.1 \\
\hline $\mathrm{FCCH}$ & $\mathrm{C}$ & 180.7 & 180.7 & 180.7 & 183.8 & 183.8 \\
\hline & $\mathrm{C}$ & 104.8 & 104.8 & 104.8 & 108.4 & 108.4 \\
\hline & $\mathrm{H}$ & 30.9 & 30.9 & 30.9 & 31.0 & 31.0 \\
\hline & $\mathrm{F}$ & 423.2 & 423.2 & 423.2 & 421.4 & 421.4 \\
\hline $\mathrm{FCN}$ & $\mathrm{F}$ & 377.9 & 377.9 & 377.9 & 376.7 & 376.7 \\
\hline & $\mathrm{C}$ & 85.4 & 85.4 & 85.4 & 92.4 & 92.4 \\
\hline & $\mathrm{N}$ & 116.7 & 116.7 & 116.7 & 131.5 & 131.5 \\
\hline $\mathrm{H}_{2} \mathrm{~S}$ & $\mathrm{~S}$ & 744.8 & 744.9 & 744.9 & 753.3 & 753.3 \\
\hline & $\mathrm{H}$ & 31.1 & 31.1 & 31.1 & 31.3 & 31.3 \\
\hline $\mathrm{HCP}$ & $\mathrm{H}$ & 30.0 & 30.0 & 30.0 & 29.9 & 29.9 \\
\hline & $\mathrm{C}$ & 38.2 & 38.2 & 38.2 & 54.1 & 54.1 \\
\hline & $\mathrm{P}$ & 394.9 & 394.9 & 394.8 & 446.8 & 446.8 \\
\hline $\mathrm{HFCO}$ & $\mathrm{O}$ & -82.9 & -82.9 & -82.9 & -54.6 & -54.6 \\
\hline & $\mathrm{C}$ & 45.0 & 45.0 & 45.0 & 53.3 & 53.3 \\
\hline & $\mathrm{F}$ & 184.0 & 184.1 & 184.1 & 184.5 & 184.5 \\
\hline & $\mathrm{H}$ & 24.6 & 24.6 & 24.6 & 24.6 & 24.6 \\
\hline $\mathrm{H}_{2} \mathrm{C}_{2} \mathrm{O}$ & $\mathrm{C}$ & 194.1 & 194.1 & 194.1 & 195.4 & 195.4 \\
\hline & $\mathrm{C}$ & 1.8 & 1.8 & 1.8 & 11.8 & 11.8 \\
\hline & $\mathrm{O}$ & 4.1 & 4.1 & 4.1 & 18.2 & 18.2 \\
\hline & $\mathrm{H}$ & 29.7 & 29.7 & 29.7 & 29.9 & 29.9 \\
\hline $\mathrm{LiF}$ & $\mathrm{Li}$ & 90.9 & 90.9 & 90.9 & 90.9 & 90.9 \\
\hline & $\mathrm{F}$ & 371.1 & 371.1 & 371.1 & 369.7 & 369.7 \\
\hline $\mathrm{LiH}$ & $\mathrm{H}$ & 26.8 & 26.8 & 26.8 & 26.5 & 26.5 \\
\hline & $\mathrm{Li}$ & 89.9 & 89.9 & 89.9 & 90.9 & 90.9 \\
\hline $\mathrm{N}_{2} \mathrm{O}$ & $\mathrm{N}$ & 107.8 & 107.8 & 107.7 & 125.4 & 125.4 \\
\hline & $\mathrm{N}$ & 13.9 & 13.9 & 13.9 & 34.5 & 34.5 \\
\hline & $\mathrm{O}$ & 197.7 & 197.7 & 197.7 & 207.2 & 207.2 \\
\hline OCS & $\mathrm{O}$ & 100.6 & 100.6 & 100.6 & 112.6 & 112.6 \\
\hline & $\mathrm{C}$ & 32.2 & 32.2 & 32.2 & 47.0 & 47.0 \\
\hline & $\mathrm{S}$ & 811.1 & 811.1 & 811.0 & 823.4 & 823.4 \\
\hline $\mathrm{OF}_{2}$ & $\mathrm{O}$ & -377.4 & -377.5 & -377.4 & -230.0 & -230.0 \\
\hline & $\mathrm{F}$ & 27.2 & 27.1 & 27.2 & 49.3 & 49.3 \\
\hline $\mathrm{H}_{4} \mathrm{C}_{2} \mathrm{O}$ & $\mathrm{O}$ & 364.7 & 364.7 & 364.7 & 359.0 & 359.0 \\
\hline & $\mathrm{C}$ & 157.5 & 157.5 & 157.5 & 159.2 & 159.2 \\
\hline & $\mathrm{H}$ & 29.7 & 29.7 & 29.7 & 29.7 & 29.7 \\
\hline $\mathrm{PN}$ & $\mathrm{N}$ & -348.5 & -348.5 & -348.5 & -268.5 & -268.5 \\
\hline & $\mathrm{P}$ & 58.4 & 58.4 & 58.4 & 177.5 & 177.5 \\
\hline
\end{tabular}


Table 13: Comparison of analytical and numerical NMR shieldings in a cc-pVTZ basis set. All values are given in ppm. The HF and PBE values were taken from Ref. 3. "HF(num.)" and "PBE(num.)" denote the numerically computed shieldings.

\begin{tabular}{|c|c|c|c|c|c|}
\hline Mol. & Nuc. & RHF & HF(num.) & $\mathrm{PBE}$ & PBE(num.) \\
\hline \multirow[t]{2}{*}{$\mathrm{HF}$} & $\mathrm{H}$ & 28.5 & 28.5 & 30.2 & 30.2 \\
\hline & $\mathrm{F}$ & 415.6 & 415.6 & 412.5 & 412.5 \\
\hline \multirow[t]{2}{*}{$\mathrm{CO}$} & $\mathrm{C}$ & -21.8 & -21.8 & -6.4 & -6.4 \\
\hline & $\mathrm{O}$ & -87.2 & -87.2 & -76.1 & -76.1 \\
\hline $\mathrm{N}_{2}$ & $\mathrm{~N}$ & -108.8 & -108.8 & -77.1 & -77.1 \\
\hline \multirow{2}{*}{$\mathrm{H}_{2} \mathrm{O}$} & $\mathrm{O}$ & 335.4 & 335.4 & 337.4 & 337.4 \\
\hline & $\mathrm{H}$ & 30.9 & 30.9 & 31.7 & 31.7 \\
\hline \multirow[t]{3}{*}{$\mathrm{HCN}$} & $\mathrm{H}$ & 29.3 & 29.3 & 29.3 & 29.3 \\
\hline & $\mathrm{C}$ & 74.4 & 74.4 & 78.2 & 78.2 \\
\hline & $\mathrm{N}$ & -47.3 & -47.3 & -37.6 & -37.6 \\
\hline \multirow{3}{*}{$\mathrm{HOF}$} & $\mathrm{O}$ & -119.5 & -119.3 & -104.9 & -104.6 \\
\hline & $\mathrm{H}$ & 19.6 & 19.6 & 20.1 & 20.1 \\
\hline & $\mathrm{F}$ & 286.6 & 286.5 & 149.2 & 149.5 \\
\hline \multirow{2}{*}{$\mathrm{NH}_{3}$} & $\mathrm{~N}$ & 268.5 & 268.5 & 269.4 & 269.4 \\
\hline & $\mathrm{H}$ & 31.9 & 31.9 & 32.2 & 32.2 \\
\hline \multirow[t]{3}{*}{$\mathrm{CH}_{2} \mathrm{O}$} & $\mathrm{O}$ & -443.9 & -443.6 & -445.7 & -445.4 \\
\hline & $\mathrm{C}$ & -2.1 & -2.1 & -15.6 & -15.6 \\
\hline & $\mathrm{H}$ & 22.7 & 22.7 & 21.0 & 21.0 \\
\hline \multirow[t]{2}{*}{$\mathrm{CH}_{4}$} & $\mathrm{C}$ & 196.7 & 196.7 & 193.6 & 193.6 \\
\hline & $\mathrm{H}$ & 31.6 & 31.6 & 31.5 & 31.5 \\
\hline \multirow[t]{2}{*}{$\mathrm{C} 2 \mathrm{H} 4$} & $\mathrm{C}$ & 63.8 & 63.8 & 56.4 & 56.4 \\
\hline & $\mathrm{H}$ & 26.3 & 26.3 & 25.7 & 25.7 \\
\hline \multirow[t]{2}{*}{$\mathrm{AlF}$} & $\mathrm{Al}$ & 583.1 & 583.1 & 544.6 & 544.6 \\
\hline & $\mathrm{F}$ & 216.3 & 216.3 & 131.4 & 131.4 \\
\hline \multirow[t]{3}{*}{$\mathrm{CH}_{3} \mathrm{~F}$} & $\mathrm{C}$ & 127.8 & 127.8 & 111.3 & 111.3 \\
\hline & $\mathrm{F}$ & 484.9 & 484.9 & 457.9 & 457.8 \\
\hline & $\mathrm{H}$ & 28.0 & 28.0 & 27.2 & 27.2 \\
\hline \multirow{4}{*}{$\mathrm{C}_{3} \mathrm{H}_{4}$} & $\mathrm{C}$ & 195.2 & 195.2 & 180.1 & 180.1 \\
\hline & $\mathrm{C}$ & 74.9 & 74.9 & 70.1 & 70.1 \\
\hline & $\mathrm{H}$ & 24.2 & 24.2 & 24.3 & 24.3 \\
\hline & $\mathrm{H}$ & 31.0 & 31.0 & 30.7 & 30.7 \\
\hline $\mathrm{FCCH}$ & $\mathrm{C}$ & 177.9 & 177.9 & 173.7 & 173.7 \\
\hline & $\mathrm{C}$ & 104.3 & 104.3 & 89.2 & 89.2 \\
\hline & $\mathrm{H}$ & 30.6 & 30.6 & 31.1 & 31.1 \\
\hline & $\mathrm{F}$ & 425.1 & 425.1 & 386.4 & 386.4 \\
\hline $\mathrm{FCN}$ & $\mathrm{F}$ & 375.2 & 375.2 & 337.7 & 337.7 \\
\hline & $\mathrm{C}$ & 78.8 & 78.8 & 75.8 & 75.8 \\
\hline & $\mathrm{N}$ & 93.3 & 93.3 & 104.0 & 104.0 \\
\hline $\mathrm{H}_{2} \mathrm{~S}$ & $\mathrm{~S}$ & 735.2 & 735.2 & 727.8 & 727.8 \\
\hline & $\mathrm{H}$ & 30.8 & 30.8 & 31.0 & 31.0 \\
\hline $\mathrm{HCP}$ & $\mathrm{H}$ & 30.2 & 30.2 & 29.6 & 29.6 \\
\hline & $\mathrm{C}$ & 17.1 & 17.1 & 22.2 & 22.2 \\
\hline & $\mathrm{P}$ & 355.1 & 355.1 & 337.8 & 337.8 \\
\hline HFCO & $\mathrm{O}$ & -126.7 & -126.6 & -119.4 & -119.4 \\
\hline & $\mathrm{C}$ & 37.3 & 37.3 & 29.6 & 29.6 \\
\hline & $\mathrm{F}$ & 188.3 & 188.3 & 93.1 & 93.1 \\
\hline & $\mathrm{H}$ & 24.6 & 24.6 & 23.6 & 23.6 \\
\hline $\mathrm{H}_{2} \mathrm{C}_{2} \mathrm{O}$ & $\mathrm{C}$ & 191.0 & 191.0 & 188.8 & 188.8 \\
\hline & $\mathrm{C}$ & -9.0 & -9.0 & -7.4 & -7.4 \\
\hline & $\mathrm{O}$ & -26.7 & -26.7 & -16.9 & -16.8 \\
\hline & $\mathrm{H}$ & 29.5 & 29.5 & 29.5 & 29.5 \\
\hline $\mathrm{LiF}$ & $\mathrm{Li}$ & 91.8 & 91.8 & 88.3 & 88.3 \\
\hline & $\mathrm{F}$ & 373.0 & 372.9 & 315.9 & 315.8 \\
\hline $\mathrm{LiH}$ & $\mathrm{H}$ & 26.5 & 26.5 & 26.3 & 26.3 \\
\hline & $\mathrm{Li}$ & 90.1 & 90.1 & 89.2 & 89.2 \\
\hline $\mathrm{N}_{2} \mathrm{O}$ & $\mathrm{N}$ & 67.1 & 67.1 & 99.3 & 99.3 \\
\hline & $\mathrm{N}$ & -27.1 & -27.1 & 12.6 & 12.6 \\
\hline & $\mathrm{O}$ & 170.4 & 170.5 & 173.6 & 173.6 \\
\hline OCS & $\mathrm{O}$ & 75.9 & 75.9 & 73.6 & 73.6 \\
\hline & $\mathrm{C}$ & 11.9 & 11.9 & 31.9 & 31.9 \\
\hline & $\mathrm{S}$ & 793.7 & 793.7 & 771.3 & 771.3 \\
\hline $\mathrm{OF}_{2}$ & $\mathrm{O}$ & -420.2 & -419.9 & -587.3 & -586.8 \\
\hline & $\mathrm{F}$ & 40.7 & 40.7 & -63.8 & -63.6 \\
\hline $\mathrm{H}_{4} \mathrm{C}_{2} \mathrm{O}$ & $\mathrm{O}$ & 377.9 & 377.9 & 330.1 & 330.1 \\
\hline & $\mathrm{C}$ & 158.7 & 158.7 & 143.0 & 143.0 \\
\hline & $\mathrm{H}$ & 29.8 & 29.8 & 29.2 & 29.2 \\
\hline $\mathrm{PN}$ & $\mathrm{N}$ & -509.2 & -509.1 & -403.9 & -403.8 \\
\hline & $\mathrm{P}$ & -91.4 & -91.2 & -26.2 & -26.1 \\
\hline
\end{tabular}


Table 14: Testing influence of number of frequency integration points in a cc-pVTZ basis set. The default of 120 grid points is used for the RPA frequency integration in "RPA@BH\&H" and " $\sigma$-P1@PBE". In the other calculations with suffix "-npX", X frequency points are employed. All values are given in ppm.

\begin{tabular}{|c|c|c|c|c|c|c|c|}
\hline$\overline{\text { Mol. }}$ & Nuc. & $\overline{\mathrm{RPA} @ \mathrm{BH} \& \mathrm{H}}$ & RPA@BH\&H-np90 & $\sigma-\mathrm{P} 1 @ \mathrm{PBE}$ & $\sigma$-P1@PBE-np160 & $\overline{\sigma \sigma \text {-P1@PBE-np200 }}$ & $\overline{\sigma \sigma-\mathrm{P} 1 @ \mathrm{PBE}-\mathrm{np} 250}$ \\
\hline \multirow[t]{2}{*}{$\mathrm{HF}$} & $\mathrm{H}$ & 29.7 & 29.7 & 30.0 & 30.0 & 30.0 & 30.0 \\
\hline & $\mathrm{F}$ & 418.6 & 418.6 & 415.7 & 415.7 & 415.7 & 415.7 \\
\hline \multirow[t]{2}{*}{$\mathrm{CO}$} & $\mathrm{C}$ & 14.3 & 14.3 & 18.4 & 18.4 & 18.7 & 18.4 \\
\hline & $\mathrm{O}$ & -37.8 & -37.8 & -36.5 & -36.5 & -36.5 & -36.4 \\
\hline $\mathrm{N}_{2}$ & $\mathrm{~N}$ & -47.3 & -47.3 & -43.0 & -43.0 & -43.0 & -43.0 \\
\hline \multirow[t]{2}{*}{$\mathrm{H}_{2} \mathrm{O}$} & $\mathrm{O}$ & 341.5 & 341.5 & 338.5 & 338.5 & 338.5 & 338.5 \\
\hline & $\mathrm{H}$ & 31.5 & 31.5 & 31.6 & 31.6 & 31.6 & 31.6 \\
\hline \multirow{3}{*}{$\mathrm{HCN}$} & $\mathrm{H}$ & 29.5 & 29.5 & 29.5 & 29.5 & 29.5 & 29.5 \\
\hline & $\mathrm{C}$ & 92.2 & 92.2 & 94.8 & 95.2 & 95.2 & 95.2 \\
\hline & $\mathrm{N}$ & -1.3 & -1.3 & 3.5 & 3.7 & 3.7 & 3.7 \\
\hline \multirow[t]{3}{*}{$\mathrm{HOF}$} & $\mathrm{O}$ & -26.7 & -26.7 & -19.6 & -19.5 & -19.6 & -19.6 \\
\hline & $\mathrm{H}$ & 21.5 & 21.5 & 21.8 & 21.8 & 21.8 & 21.8 \\
\hline & $\mathrm{F}$ & 209.9 & 209.9 & 190.4 & 190.4 & 190.4 & 190.4 \\
\hline \multirow[t]{2}{*}{$\mathrm{NH}_{3}$} & $\mathrm{~N}$ & 273.0 & 273.0 & 271.3 & 271.3 & 271.3 & 271.3 \\
\hline & $\mathrm{H}$ & 32.2 & 32.2 & 32.1 & 32.1 & 32.1 & 32.1 \\
\hline \multirow[t]{3}{*}{$\mathrm{CH}_{2} \mathrm{O}$} & $\mathrm{O}$ & -316.4 & -316.4 & -333.6 & -333.5 & -333.5 & -333.5 \\
\hline & $\mathrm{C}$ & 18.6 & 18.6 & 24.1 & 24.1 & 23.9 & 24.1 \\
\hline & $\mathrm{H}$ & 22.9 & 22.9 & 23.0 & 23.0 & 23.0 & 23.0 \\
\hline \multirow[t]{2}{*}{$\mathrm{CH}_{4}$} & $\mathrm{C}$ & 198.9 & 198.9 & 198.4 & 198.4 & 198.4 & 198.4 \\
\hline & $\mathrm{H}$ & 31.6 & 31.6 & 31.6 & 31.6 & 31.6 & 31.6 \\
\hline \multirow[t]{2}{*}{$\mathrm{C} 2 \mathrm{H} 4$} & $\mathrm{C}$ & 77.6 & 77.6 & 80.8 & 80.8 & 80.8 & 80.8 \\
\hline & $\mathrm{H}$ & 26.6 & 26.6 & 26.7 & 26.7 & 26.7 & 26.7 \\
\hline \multirow[t]{2}{*}{$\mathrm{AlF}$} & $\mathrm{Al}$ & 580.7 & 580.7 & 583.0 & 583.0 & 583.0 & 583.0 \\
\hline & $\mathrm{F}$ & 216.0 & 216.0 & 214.0 & 214.0 & 214.1 & 214.0 \\
\hline \multirow[t]{3}{*}{$\mathrm{CH}_{3} \mathrm{~F}$} & $\mathrm{C}$ & 128.1 & 128.1 & 128.8 & 128.8 & 128.8 & 128.8 \\
\hline & $\mathrm{F}$ & 474.5 & 474.5 & 475.4 & 475.4 & 475.3 & 475.3 \\
\hline & $\mathrm{H}$ & 27.9 & 27.9 & 28.1 & 28.1 & 28.1 & 28.1 \\
\hline $\mathrm{C}_{3} \mathrm{H}_{4}$ & $\mathrm{C}$ & 193.5 & 193.5 & 194.3 & 194.2 & 194.2 & 194.2 \\
\hline & $\mathrm{C}$ & 90.8 & 90.8 & 94.1 & 94.1 & 94.1 & 94.1 \\
\hline & $\mathrm{H}$ & 25.0 & 25.0 & 25.1 & 25.1 & 25.1 & 25.1 \\
\hline & $\mathrm{H}$ & 31.1 & 31.1 & 31.2 & 31.2 & 31.2 & 31.2 \\
\hline $\mathrm{FCCH}$ & $\mathrm{C}$ & 182.7 & 182.7 & 182.5 & 182.5 & 182.6 & 182.5 \\
\hline & $\mathrm{C}$ & 106.4 & 106.4 & 107.6 & 107.6 & 107.6 & 107.6 \\
\hline & $\mathrm{H}$ & 30.9 & 30.9 & 31.0 & 31.0 & 31.0 & 31.0 \\
\hline & $\mathrm{F}$ & 422.5 & 422.5 & 421.7 & 421.7 & 421.7 & 421.7 \\
\hline $\mathrm{FCN}$ & $\mathrm{F}$ & 376.7 & 376.7 & 373.6 & 373.6 & 373.2 & 373.6 \\
\hline & $\mathrm{C}$ & 88.8 & 88.8 & 90.5 & 90.5 & 90.5 & 90.5 \\
\hline & $\mathrm{N}$ & 125.1 & 125.1 & 125.6 & 125.6 & 125.6 & 125.6 \\
\hline $\mathrm{H}_{2} \mathrm{~S}$ & $\mathrm{~S}$ & 747.7 & 747.7 & 739.5 & 739.6 & 739.5 & 739.6 \\
\hline & $\mathrm{H}$ & 31.2 & 31.2 & 31.4 & 31.4 & 31.4 & 31.4 \\
\hline $\mathrm{HCP}$ & $\mathrm{H}$ & 30.0 & 30.0 & 30.1 & 30.1 & 30.1 & 30.1 \\
\hline & $\mathrm{C}$ & 45.7 & 45.7 & 52.5 & 52.5 & 52.5 & 52.5 \\
\hline & $\mathrm{P}$ & 416.8 & 416.8 & 436.5 & 436.5 & 436.5 & 436.5 \\
\hline $\mathrm{HFCO}$ & $\mathrm{O}$ & -65.9 & -65.9 & -76.7 & -76.7 & -76.7 & -76.7 \\
\hline & $\mathrm{C}$ & 48.8 & 48.8 & 49.9 & 49.9 & 49.9 & 49.9 \\
\hline & $\mathrm{F}$ & 181.6 & 181.6 & 174.4 & 174.4 & 174.3 & 174.4 \\
\hline & $\mathrm{H}$ & 24.6 & 24.6 & 24.6 & 24.6 & 24.6 & 24.6 \\
\hline $\mathrm{H}_{2} \mathrm{C}_{2} \mathrm{O}$ & $\mathrm{C}$ & 194.9 & 194.9 & 194.0 & 193.9 & 194.0 & 194.0 \\
\hline & $\mathrm{C}$ & 6.6 & 6.6 & 5.3 & 5.3 & 5.3 & 5.4 \\
\hline & $\mathrm{O}$ & 13.5 & 13.5 & 3.1 & 3.2 & 3.2 & 3.2 \\
\hline & $\mathrm{H}$ & 29.8 & 29.8 & 29.8 & 29.8 & 29.8 & 29.8 \\
\hline $\mathrm{LiF}$ & $\mathrm{Li}$ & 90.8 & 90.8 & 90.8 & 90.8 & 90.8 & 90.8 \\
\hline & $\mathrm{F}$ & 370.6 & 370.6 & 369.7 & 369.7 & 369.7 & 369.7 \\
\hline $\mathrm{LiH}$ & $\mathrm{H}$ & 26.8 & 26.8 & 26.7 & 26.7 & 26.7 & 26.7 \\
\hline & $\mathrm{Li}$ & 90.2 & 90.2 & 90.6 & 90.6 & 90.6 & 90.6 \\
\hline $\mathrm{N}_{2} \mathrm{O}$ & $\mathrm{N}$ & 116.2 & 116.2 & 118.0 & 118.4 & 118.0 & 118.0 \\
\hline & $\mathrm{N}$ & 23.3 & 23.3 & 29.7 & 29.7 & 29.7 & 29.7 \\
\hline & $\mathrm{O}$ & 202.6 & 202.6 & 200.0 & 200.0 & 200.0 & 202.5 \\
\hline OCS & $\mathrm{O}$ & 106.6 & 106.6 & 108.6 & 108.4 & 108.4 & 108.1 \\
\hline & $\mathrm{C}$ & 39.3 & 39.3 & 44.2 & 44.2 & 44.2 & 44.0 \\
\hline & $\mathrm{S}$ & 816.6 & 816.6 & 811.8 & 811.8 & 811.8 & 811.8 \\
\hline $\mathrm{OF}_{2}$ & $\mathrm{O}$ & -356.8 & -356.8 & -341.9 & -341.6 & -341.3 & -341.9 \\
\hline & $\mathrm{F}$ & 31.1 & 31.1 & 12.4 & 12.4 & 12.8 & 12.3 \\
\hline $\mathrm{H}_{4} \mathrm{C}_{2} \mathrm{O}$ & $\mathrm{O}$ & 362.9 & 362.9 & 359.3 & 359.3 & 359.3 & 359.3 \\
\hline & $\mathrm{C}$ & 158.0 & 158.0 & 158.3 & 158.3 & 158.3 & 158.3 \\
\hline & $\mathrm{H}$ & 29.7 & 29.7 & 29.7 & 29.7 & 29.7 & 29.7 \\
\hline $\mathrm{PN}$ & $\mathrm{N}$ & -317.7 & -317.7 & -299.1 & -299.1 & -299.1 & -299.0 \\
\hline & $\mathrm{P}$ & 100.8 & 100.8 & 132.7 & 132.3 & 132.7 & 132.7 \\
\hline
\end{tabular}


Table 15: Absolute isotropic NMR shieldings in a cc-pVQZ basis. All values are given in ppm. "Extrap." stands for basis-set extrapolated $\operatorname{CCSD}(\mathrm{T})$-shieldings in an aug-cc-pCV[TQ]Z basis set. The data for "Extrap." was taken from Ref. 3, CCSD(T) calculations were done with the CFOUR program [1, 2]. MP2 calculations were done with the Turbomole program [4 [6].

\begin{tabular}{|c|c|c|c|c|c|c|c|}
\hline Mol. & Nuc. & $\operatorname{CCSD}(\mathrm{T})$ & Extrap. & RPA@HF & RPA@PBE & $\sigma$-P1@PBE & MP2 \\
\hline \multirow[t]{2}{*}{$\mathrm{HF}$} & $\mathrm{H}$ & 29.1 & 28.8 & 29.0 & 29.6 & 29.1 & 28.8 \\
\hline & $\mathrm{F}$ & 419.9 & 420.3 & 419.2 & 418.5 & 418.3 & 425.3 \\
\hline \multirow[t]{2}{*}{$\mathrm{CO}$} & $\mathrm{C}$ & 5.9 & 2.2 & -0.5 & 22.4 & 11.5 & 11.2 \\
\hline & $\mathrm{O}$ & -51.9 & -55.0 & -50.7 & -25.1 & -38.9 & -45.2 \\
\hline $\mathrm{N}_{2}$ & $\mathrm{~N}$ & -57.6 & -60.4 & -64.3 & -34.7 & -49.5 & -40.6 \\
\hline \multirow[t]{2}{*}{$\mathrm{H}_{2} \mathrm{O}$} & $\mathrm{O}$ & 341.0 & 338.0 & 338.5 & 338.5 & 338.0 & 349.1 \\
\hline & $\mathrm{H}$ & 30.9 & 30.6 & 30.9 & 31.2 & 30.9 & 30.6 \\
\hline \multirow[t]{3}{*}{$\mathrm{HCN}$} & $\mathrm{H}$ & 29.1 & 29.0 & 29.3 & 29.3 & 29.3 & 29.0 \\
\hline & $\mathrm{C}$ & 87.3 & 84.6 & 83.3 & 94.4 & 90.1 & 88.7 \\
\hline & $\mathrm{N}$ & -11.3 & -14.1 & -15.9 & 8.2 & -1.3 & 1.6 \\
\hline \multirow{3}{*}{$\mathrm{HOF}$} & $\mathrm{O}$ & -58.7 & -68.9 & -57.7 & 8.6 & -37.1 & -43.9 \\
\hline & $\mathrm{H}$ & 20.0 & 19.6 & 20.6 & 22.0 & 20.7 & 20.1 \\
\hline & $\mathrm{F}$ & 191.6 & 192.2 & 215.3 & 214.2 & 194.7 & 194.9 \\
\hline \multirow[t]{2}{*}{$\mathrm{NH}_{3}$} & $\mathrm{~N}$ & 273.1 & 270.7 & 269.8 & 270.6 & 269.5 & 278.7 \\
\hline & $\mathrm{H}$ & 31.7 & 31.4 & 31.8 & 31.9 & 31.7 & 31.5 \\
\hline \multirow{3}{*}{$\mathrm{CH}_{2} \mathrm{O}$} & $\mathrm{O}$ & -377.2 & -378.6 & -350.5 & -271.3 & -333.0 & -332.6 \\
\hline & $\mathrm{C}$ & 6.5 & 1.5 & 5.7 & 24.7 & 15.3 & 6.6 \\
\hline & $\mathrm{H}$ & 22.1 & 22.0 & 22.6 & 23.0 & 22.6 & 22.1 \\
\hline \multirow[t]{2}{*}{$\mathrm{CH}_{4}$} & $\mathrm{C}$ & 199.9 & 198.9 & 196.9 & 197.9 & 198.0 & 201.5 \\
\hline & $\mathrm{H}$ & 31.4 & 31.3 & 31.5 & 31.5 & 31.4 & 31.3 \\
\hline \multirow[t]{2}{*}{$\mathrm{C} 2 \mathrm{H} 4$} & $\mathrm{C}$ & 72.9 & 69.7 & 69.0 & 78.7 & 75.7 & 71.4 \\
\hline & $\mathrm{H}$ & 26.1 & 26.0 & 26.4 & 26.5 & 26.4 & 26.0 \\
\hline \multirow[t]{2}{*}{$\mathrm{AlF}$} & $\mathrm{Al}$ & 587.7 & 572.9 & 589.8 & 589.7 & 587.5 & 587.0 \\
\hline & $\mathrm{F}$ & 212.2 & 211.8 & 228.7 & 229.5 & 231.0 & 209.5 \\
\hline \multirow{3}{*}{$\mathrm{CH}_{3} \mathrm{~F}$} & $\mathrm{C}$ & 124.5 & 122.1 & 124.6 & 127.3 & 126.1 & 123.0 \\
\hline & $\mathrm{F}$ & 482.8 & 482.9 & 480.2 & 475.6 & 481.1 & 488.9 \\
\hline & $\mathrm{H}$ & 27.5 & 27.3 & 27.8 & 27.8 & 27.7 & 27.4 \\
\hline $\mathrm{C}_{3} \mathrm{H}_{4}$ & $\mathrm{C}$ & 193.2 & 192.1 & 191.4 & 192.4 & 192.4 & 194.1 \\
\hline & $\mathrm{C}$ & 86.5 & 83.7 & 83.1 & 92.5 & 89.7 & 87.7 \\
\hline & $\mathrm{H}$ & 24.5 & 24.4 & 24.6 & 24.9 & 24.8 & 24.4 \\
\hline & $\mathrm{H}$ & 30.7 & 30.6 & 30.9 & 30.9 & 30.9 & 30.7 \\
\hline $\mathrm{FCCH}$ & $\mathrm{C}$ & 181.4 & 179.9 & 179.0 & 181.9 & 179.6 & 183.2 \\
\hline & $\mathrm{C}$ & 102.6 & 100.1 & 102.3 & 106.0 & 104.8 & 99.9 \\
\hline & $\mathrm{H}$ & 30.6 & 30.5 & 30.7 & 30.8 & 30.6 & 30.5 \\
\hline & $\mathrm{F}$ & 424.0 & 423.5 & 425.4 & 423.0 & 424.9 & 430.0 \\
\hline $\mathrm{FCN}$ & $\mathrm{F}$ & 375.1 & 374.1 & 379.2 & 377.3 & 376.4 & 383.7 \\
\hline & $\mathrm{C}$ & 84.8 & 82.2 & 82.0 & 89.1 & 86.6 & 83.7 \\
\hline & $\mathrm{N}$ & 120.1 & 117.9 & 114.1 & 128.8 & 122.1 & 133.1 \\
\hline $\mathrm{H}_{2} \mathrm{~S}$ & $\mathrm{~S}$ & 759.6 & 739.0 & 744.2 & 752.4 & 743.9 & 775.1 \\
\hline & $\mathrm{H}$ & 30.7 & 30.5 & 30.9 & 31.0 & 30.9 & 30.5 \\
\hline $\mathrm{HCP}$ & $\mathrm{H}$ & 29.7 & 29.6 & 30.0 & 29.9 & 29.9 & 29.5 \\
\hline & $\mathrm{C}$ & 41.1 & 37.6 & 34.0 & 49.5 & 46.4 & 45.8 \\
\hline & $\mathrm{P}$ & 426.9 & 388.0 & 408.5 & 443.1 & 432.9 & 432.6 \\
\hline $\mathrm{HFCO}$ & $\mathrm{O}$ & -91.0 & -94.3 & -85.7 & -59.0 & -79.2 & -60.2 \\
\hline & $\mathrm{C}$ & 43.1 & 39.6 & 41.1 & 49.2 & 44.9 & 43.4 \\
\hline & $\mathrm{F}$ & 166.9 & 165.3 & 183.7 & 183.9 & 177.2 & 166.6 \\
\hline & $\mathrm{H}$ & 24.0 & 23.9 & 24.3 & 24.3 & 24.2 & 23.9 \\
\hline $\mathrm{H}_{2} \mathrm{C}_{2} \mathrm{O}$ & $\mathrm{C}$ & 194.4 & 193.3 & 192.0 & 193.6 & 192.4 & 199.1 \\
\hline & $\mathrm{C}$ & -2.0 & -6.3 & -3.9 & 6.1 & -0.7 & 1.7 \\
\hline & $\mathrm{O}$ & -4.3 & -5.9 & 3.1 & 16.6 & 4.7 & 29.1 \\
\hline & $\mathrm{H}$ & 29.3 & 29.2 & 29.5 & 29.6 & 29.4 & 29.4 \\
\hline $\mathrm{LiF}$ & $\mathrm{Li}$ & 89.3 & 89.3 & 89.7 & 89.7 & 89.5 & 89.3 \\
\hline & $\mathrm{F}$ & 375.8 & 382.5 & 383.6 & 383.8 & 387.9 & 373.9 \\
\hline $\mathrm{LiH}$ & $\mathrm{H}$ & 26.6 & 26.6 & 26.9 & 26.6 & 26.8 & 26.7 \\
\hline & $\mathrm{Li}$ & 89.3 & 89.3 & 89.2 & 90.2 & 89.4 & 89.3 \\
\hline $\mathrm{N}_{2} \mathrm{O}$ & $\mathrm{N}$ & 109.4 & 106.4 & 102.0 & 120.1 & 112.3 & 132.0 \\
\hline & $\mathrm{N}$ & 16.0 & 12.6 & 7.5 & 28.0 & 23.1 & 33.6 \\
\hline & $\mathrm{O}$ & 199.9 & 199.0 & 199.3 & 208.3 & 201.7 & 217.4 \\
\hline OCS & $\mathrm{O}$ & 98.7 & 96.8 & 100.3 & 111.7 & 108.8 & 111.9 \\
\hline & $\mathrm{C}$ & 33.8 & 30.2 & 27.9 & 42.5 & 39.5 & 40.7 \\
\hline & $\mathrm{S}$ & 815.1 & 796.7 & 814.1 & 819.1 & 810.1 & 833.9 \\
\hline $\mathrm{OF}_{2}$ & $\mathrm{O}$ & -430.3 & -447.1 & -406.1 & -260.8 & -374.3 & -451.6 \\
\hline & $\mathrm{F}$ & -12.7 & -24.0 & 14.0 & 34.7 & -0.5 & -3.4 \\
\hline $\mathrm{H}_{4} \mathrm{C}_{2} \mathrm{O}$ & $\mathrm{O}$ & 364.7 & 363.2 & 366.5 & 360.2 & 360.7 & 373.5 \\
\hline & $\mathrm{C}$ & 155.1 & 153.2 & 154.5 & 156.4 & 155.1 & 154.2 \\
\hline & $\mathrm{H}$ & 29.2 & 29.1 & 29.5 & 29.4 & 29.4 & 29.2 \\
\hline $\mathrm{PN}$ & $\mathrm{N}$ & -340.7 & -344.0 & -353.8 & -276.3 & -306.7 & -252.2 \\
\hline & $\mathrm{P}$ & 118.2 & 50.6 & 84.8 & 191.2 & 149.7 & 176.7 \\
\hline
\end{tabular}


Table 16: Statistics for absolute isotropic NMR shieldings in a cc-pVQZ basis. CCSD(T)/aug-cc$\mathrm{pCV}[\mathrm{TQ}] \mathrm{Z}$ shieldings are used as a reference. $\mathrm{MSD}=$ mean signed deviation; $\mathrm{MAD}=\mathrm{mean}$ absolute deviation; $\mathrm{MRD}=$ mean relative deviation [\%]; $\mathrm{MARD}=$ mean absolute relative deviation [\%]; $\mathrm{RMSD}=\mathrm{root}$ mean squared deviation.

\begin{tabular}{lrrrrr}
\hline & MSD & MAD & MRD & MARD & RMSD \\
\hline RHF & -9.2 & 17.3 & -49.9 & 59.8 & 32.9 \\
PBE & -19.6 & 19.8 & -58.9 & 59.3 & 32.6 \\
KT2 & -5.3 & 9.4 & 5.8 & 18.4 & 17.3 \\
B3LYP & -20.0 & 20.2 & -69.9 & 70.4 & 30.7 \\
CCSD & 3.4 & 4.9 & 5.7 & 11.1 & 10.2 \\
CCSD(T) & 4.1 & 4.3 & 13.9 & 14.0 & 10.8 \\
RPA@HF & 4.1 & 5.6 & 9.0 & 15.4 & 10.8 \\
RPA@BH\&H & 8.5 & 8.8 & 30.7 & 30.8 & 17.3 \\
RPA@B3LYP & 12.3 & 12.6 & 48.3 & 48.4 & 25.0 \\
RPA@KT2 & 13.1 & 13.8 & 51.7 & 51.9 & 27.7 \\
RPA@PBE & 16.9 & 17.3 & 65.7 & 65.8 & 36.7 \\
$\sigma-P 1 @ P B E$ & 9.0 & 9.3 & 35.5 & 35.6 & 19.2 \\
MP2 & 11.3 & 11.8 & 35.8 & 36.0 & 23.8 \\
\hline \hline
\end{tabular}

Table 17: Statistics for absolute isotropic NMR shieldings in a cc-pVQZ basis. CCSD(T) shieldings are used as a reference. $\mathrm{MSD}=$ mean signed deviation; $\mathrm{MAD}=$ mean absolute deviation; $\mathrm{MRD}=$ mean relative deviation [\%]; MARD=mean absolute relative deviation [\%]; RMSD=root mean squared deviation.

\begin{tabular}{lrrrrr}
\hline & MSD & MAD & MRD & MARD & RMSD \\
\hline RHF & -13.4 & 20.1 & -43.5 & 56.2 & 37.9 \\
PBE & -23.8 & 23.8 & -52.5 & 52.8 & 38.5 \\
KT2 & -9.4 & 10.6 & -4.5 & 17.9 & 19.4 \\
B3LYP & -24.1 & 24.2 & -63.9 & 64.1 & 37.3 \\
CCSD & -0.7 & 3.4 & -5.0 & 9.2 & 6.4 \\
CCSD(T) & 0.0 & 0.0 & 0.0 & 0.0 & 0.0 \\
RPA@HF & -0.0 & 5.1 & 0.3 & 13.2 & 9.1 \\
RPA@BH\&H & 4.4 & 5.3 & 16.4 & 16.7 & 11.6 \\
RPA@B3LYP & 8.2 & 9.0 & 28.3 & 28.5 & 18.8 \\
RPA@KT2 & 9.0 & 10.0 & 30.5 & 30.8 & 20.5 \\
RPA@PBE & 12.8 & 13.6 & 39.0 & 39.2 & 30.3 \\
$\sigma-P 1 @ P B E$ & 4.9 & 6.1 & 14.3 & 14.6 & 12.0 \\
MP2 & 7.2 & 8.3 & 24.0 & 24.6 & 16.8 \\
\hline \hline
\end{tabular}


Table 18: Absolute isotropic NMR shieldings in a cc-pCVDZ basis. All values are given in ppm. "Extrap." stands for basis-set extrapolated CCSD(T)-shieldings in an aug-cc-pCV[TQ]Z basis set. The data for "Extrap." was taken from Ref. 3. CCSD(T) calculations were done with the CFOUR program[1, [2].

\begin{tabular}{|c|c|c|c|c|c|c|}
\hline Mol. & Nuc. & $\operatorname{CCSD}(\mathrm{T})$ & Extrap. & RPA@HF & RPA@PBE & $\sigma-\mathrm{P} 1 @ \mathrm{PBE}$ \\
\hline \multirow[t]{2}{*}{$\mathrm{HF}$} & $\mathrm{H}$ & 30.5 & 28.8 & 30.4 & 31.1 & 31.4 \\
\hline & $\mathrm{F}$ & 426.0 & 420.3 & 426.2 & 424.0 & 420.7 \\
\hline \multirow[t]{2}{*}{$\mathrm{CO}$} & $\mathrm{C}$ & 28.9 & 2.2 & 27.0 & 52.2 & 42.8 \\
\hline & $\mathrm{O}$ & -24.3 & -55.0 & -18.2 & 12.1 & -7.3 \\
\hline $\mathrm{N}_{2}$ & $\mathrm{~N}$ & -32.8 & -60.4 & -32.7 & -1.8 & -18.4 \\
\hline \multirow[t]{2}{*}{$\mathrm{H}_{2} \mathrm{O}$} & $\mathrm{O}$ & 353.7 & 338.0 & 352.5 & 350.5 & 346.2 \\
\hline & $\mathrm{H}$ & 31.9 & 30.6 & 31.9 & 32.3 & 32.5 \\
\hline \multirow[t]{3}{*}{$\mathrm{HCN}$} & $\mathrm{H}$ & 29.4 & 29.0 & 29.5 & 29.6 & 29.6 \\
\hline & $\mathrm{C}$ & 106.3 & 84.6 & 103.6 & 116.0 & 113.0 \\
\hline & $\mathrm{N}$ & 13.9 & -14.1 & 12.8 & 39.4 & 31.5 \\
\hline \multirow[t]{3}{*}{$\mathrm{HOF}$} & $\mathrm{O}$ & -12.4 & -68.9 & 2.6 & 72.9 & 38.1 \\
\hline & $\mathrm{H}$ & 21.8 & 19.6 & 22.6 & 24.5 & 23.8 \\
\hline & $\mathrm{F}$ & 179.7 & 192.2 & 204.4 & 219.7 & 210.3 \\
\hline \multirow[t]{2}{*}{$\mathrm{NH}_{3}$} & $\mathrm{~N}$ & 282.2 & 270.7 & 280.1 & 279.5 & 276.8 \\
\hline & $\mathrm{H}$ & 32.3 & 31.4 & 32.4 & 32.5 & 32.6 \\
\hline \multirow[t]{3}{*}{$\mathrm{CH}_{2} \mathrm{O}$} & $\mathrm{O}$ & -352.1 & -378.6 & -321.4 & -212.0 & -268.7 \\
\hline & $\mathrm{C}$ & 32.3 & 1.5 & 32.7 & 56.4 & 51.0 \\
\hline & $\mathrm{H}$ & 22.6 & 22.0 & 23.1 & 23.7 & 23.4 \\
\hline \multirow{2}{*}{$\mathrm{CH}_{4}$} & $\mathrm{C}$ & 206.8 & 198.9 & 204.9 & 206.4 & 204.5 \\
\hline & $\mathrm{H}$ & 31.6 & 31.3 & 31.7 & 31.7 & 31.7 \\
\hline \multirow[t]{2}{*}{$\mathrm{C} 2 \mathrm{H} 4$} & $\mathrm{C}$ & 90.3 & 69.7 & 87.2 & 99.2 & 98.3 \\
\hline & $\mathrm{H}$ & 26.6 & 26.0 & 26.7 & 27.0 & 27.0 \\
\hline \multirow[t]{2}{*}{$\mathrm{AlF}$} & $\mathrm{Al}$ & 578.6 & 572.9 & 584.9 & 592.8 & 592.1 \\
\hline & $\mathrm{F}$ & 186.3 & 211.8 & 207.7 & 203.7 & 199.8 \\
\hline \multirow[t]{3}{*}{$\mathrm{CH}_{3} \mathrm{~F}$} & $\mathrm{C}$ & 139.1 & 122.1 & 139.3 & 144.1 & 142.7 \\
\hline & $\mathrm{F}$ & 475.6 & 482.9 & 476.4 & 469.8 & 470.1 \\
\hline & $\mathrm{H}$ & 27.9 & 27.3 & 28.1 & 28.2 & 28.3 \\
\hline $\mathrm{C}_{3} \mathrm{H}_{4}$ & $\mathrm{C}$ & 202.2 & 192.1 & 201.2 & 203.2 & 202.9 \\
\hline & $\mathrm{C}$ & 102.7 & 83.7 & 100.9 & 111.8 & 109.9 \\
\hline & $\mathrm{H}$ & 25.0 & 24.4 & 25.2 & 25.6 & 25.5 \\
\hline & $\mathrm{H}$ & 31.1 & 30.6 & 31.3 & 31.3 & 31.3 \\
\hline $\mathrm{FCCH}$ & $\mathrm{C}$ & 189.6 & 179.9 & 188.0 & 192.3 & 193.2 \\
\hline & $\mathrm{C}$ & 120.7 & 100.1 & 120.5 & 125.6 & 125.0 \\
\hline & $\mathrm{H}$ & 31.0 & 30.5 & 31.0 & 31.2 & 31.2 \\
\hline & $\mathrm{F}$ & 428.0 & 423.5 & 431.4 & 429.6 & 430.0 \\
\hline $\mathrm{FCN}$ & $\mathrm{F}$ & 382.5 & 374.1 & 389.3 & 388.5 & 384.2 \\
\hline & $\mathrm{C}$ & 105.7 & 82.2 & 103.7 & 111.9 & 110.4 \\
\hline & $\mathrm{N}$ & 135.8 & 117.9 & 133.3 & 149.8 & 147.3 \\
\hline $\mathrm{H}_{2} \mathrm{~S}$ & $\mathrm{~S}$ & 756.4 & 739.0 & 750.5 & 759.2 & 745.5 \\
\hline & $\mathrm{H}$ & 31.3 & 30.5 & 31.4 & 31.6 & 31.8 \\
\hline $\mathrm{HCP}$ & $\mathrm{H}$ & 29.4 & 29.6 & 29.6 & 29.6 & 29.7 \\
\hline & $\mathrm{C}$ & 61.6 & 37.6 & 55.5 & 74.2 & 72.3 \\
\hline & $\mathrm{P}$ & 422.0 & 388.0 & 407.3 & 465.8 & 462.4 \\
\hline $\mathrm{HFCO}$ & $\mathrm{O}$ & -67.1 & -94.3 & -56.5 & -22.1 & -40.7 \\
\hline & $\mathrm{C}$ & 64.0 & 39.6 & 63.1 & 73.9 & 71.1 \\
\hline & $\mathrm{F}$ & 183.1 & 165.3 & 200.7 & 204.0 & 193.1 \\
\hline & $\mathrm{H}$ & 24.7 & 23.9 & 24.9 & 25.0 & 24.9 \\
\hline $\mathrm{H}_{2} \mathrm{C}_{2} \mathrm{O}$ & $\mathrm{C}$ & 202.2 & 193.3 & 201.4 & 203.1 & 202.3 \\
\hline & $\mathrm{C}$ & 21.2 & -6.3 & 21.8 & 33.6 & 26.6 \\
\hline & $\mathrm{O}$ & 14.8 & -5.9 & 29.1 & 44.6 & 27.7 \\
\hline & $\mathrm{H}$ & 30.0 & 29.2 & 30.2 & 30.3 & 30.4 \\
\hline $\mathrm{LiF}$ & $\mathrm{Li}$ & 93.0 & 89.3 & 93.2 & 93.4 & 93.5 \\
\hline & $\mathrm{F}$ & 348.3 & 382.5 & 358.8 & 352.2 & 354.5 \\
\hline $\mathrm{LiH}$ & $\mathrm{H}$ & 26.3 & 26.6 & 26.5 & 26.2 & 26.3 \\
\hline & $\mathrm{Li}$ & 91.7 & 89.3 & 91.8 & 93.2 & 93.4 \\
\hline $\mathrm{N}_{2} \mathrm{O}$ & $\mathrm{N}$ & 125.2 & 106.4 & 123.3 & 140.8 & 135.9 \\
\hline & $\mathrm{N}$ & 41.3 & 12.6 & 36.6 & 57.0 & 51.0 \\
\hline & $\mathrm{O}$ & 206.2 & 199.0 & 211.6 & 220.3 & 213.7 \\
\hline OCS & $\mathrm{O}$ & 117.0 & 96.8 & 121.8 & 135.6 & 127.3 \\
\hline & $\mathrm{C}$ & 54.4 & 30.2 & 50.6 & 66.5 & 63.2 \\
\hline & S & 817.4 & 796.7 & 823.6 & 836.3 & 830.8 \\
\hline $\mathrm{OF}_{2}$ & $\mathrm{O}$ & -349.1 & -447.1 & -314.6 & -132.6 & -234.1 \\
\hline & $\mathrm{F}$ & 13.9 & -24.0 & 45.6 & 77.1 & 55.3 \\
\hline $\mathrm{H}_{4} \mathrm{C}_{2} \mathrm{O}$ & $\mathrm{O}$ & 364.0 & 363.2 & 368.9 & 364.1 & 363.5 \\
\hline & $\mathrm{C}$ & 167.5 & 153.2 & 167.5 & 170.8 & 170.1 \\
\hline & $\mathrm{H}$ & 29.7 & 29.1 & 30.0 & 29.9 & 30.0 \\
\hline $\mathrm{PN}$ & $\mathrm{N}$ & -295.2 & -344.0 & -289.6 & -200.5 & -252.9 \\
\hline & $\mathrm{P}$ & 111.2 & 50.6 & 100.4 & 235.3 & 168.0 \\
\hline
\end{tabular}


Table 19: Statistics for absolute isotropic NMR shieldings in a cc-pCVDZ basis. CCSD(T)/aug-cc$\mathrm{pCV}[\mathrm{TQ}] \mathrm{Z}$ shieldings are used as a reference. $\mathrm{MSD}=$ mean signed deviation; $\mathrm{MAD}=$ mean absolute deviation; $\mathrm{MRD}=$ mean relative deviation [\%]; $\mathrm{MARD}=$ mean absolute relative deviation [\%]; $\mathrm{RMSD}=$ root mean squared deviation.

\begin{tabular}{lrrrrr}
\hline \hline & MSD & MAD & MRD & MARD & RMSD \\
\hline RHF & -4.0 & 17.1 & -6.4 & 43.2 & 34.8 \\
CCSD & 14.0 & 15.4 & 74.2 & 74.7 & 23.6 \\
CCSD(T) & 14.2 & 16.6 & 81.1 & 82.0 & 23.8 \\
RPA@HF & 17.1 & 18.1 & 85.9 & 86.2 & 28.2 \\
RPA@PBE & 33.1 & 34.6 & 152.9 & 153.4 & 62.4 \\
$\sigma-\mathrm{P} 1 @ P B E$ & 25.1 & 26.6 & 126.8 & 127.3 & 44.5 \\
\hline \hline
\end{tabular}

Table 20: Statistics for absolute isotropic NMR shieldings in a cc-pCVDZ basis. CCSD(T) shieldings are used as a reference. $\mathrm{MSD}=$ mean signed deviation; $\mathrm{MAD}=$ mean absolute deviation; $\mathrm{MRD}=$ mean relative deviation [\%]; MARD=mean absolute relative deviation [\%]; RMSD=root mean squared deviation.

\begin{tabular}{lrrrrr}
\hline \hline & MSD & MAD & MRD & MARD & RMSD \\
\hline RHF & -18.2 & 24.9 & -35.4 & 45.2 & 47.5 \\
CCSD & -0.3 & 3.0 & -0.5 & 4.6 & 5.7 \\
CCSD(T) & 0.0 & 0.0 & 0.0 & 0.0 & 0.0 \\
RPA@HF & 2.9 & 5.0 & 7.4 & 9.6 & 9.4 \\
RPA@PBE & 18.8 & 19.3 & 37.9 & 38.0 & 41.3 \\
$\sigma$-P1@PBE & 10.9 & 12.0 & 22.5 & 22.8 & 23.1 \\
\hline
\end{tabular}


Table 21: Absolute isotropic NMR shieldings in a cc-pCVTZ basis. All values are given in ppm. "Extrap." stands for basis-set extrapolated $\operatorname{CCSD}(\mathrm{T})$-shieldings in an aug-cc-pCV[TQ]Z basis set. The data for "Extrap." was taken from Ref. 3. CCSD(T) calculations were done with the CFOUR program[1, 2.

\begin{tabular}{|c|c|c|c|c|c|c|}
\hline Mol. & Nuc. & $\overline{\mathrm{CCSD}(\mathrm{T})}$ & Extrap. & $\overline{\mathrm{RPA} @ H F}$ & RPA@PBE & $\sigma-\mathrm{P} 1 @ \mathrm{PBE}$ \\
\hline \multirow[t]{2}{*}{$\mathrm{HF}$} & $\mathrm{H}$ & 29.4 & 28.8 & 29.4 & 30.0 & 29.9 \\
\hline & $\mathrm{F}$ & 420.4 & 420.3 & 419.5 & 418.2 & 415.9 \\
\hline \multirow[t]{2}{*}{$\mathrm{CO}$} & $\mathrm{C}$ & 8.0 & 2.2 & 2.2 & 26.0 & 16.1 \\
\hline & $\mathrm{O}$ & -52.0 & -55.0 & -51.3 & -24.0 & -39.8 \\
\hline $\mathrm{N}_{2}$ & $\mathrm{~N}$ & -56.3 & -60.4 & -62.9 & -32.3 & -47.5 \\
\hline \multirow[t]{2}{*}{$\mathrm{H}_{2} \mathrm{O}$} & $\mathrm{O}$ & 344.5 & 338.0 & 341.8 & 341.1 & 338.3 \\
\hline & $\mathrm{H}$ & 31.2 & 30.6 & 31.3 & 31.6 & 31.5 \\
\hline \multirow[t]{3}{*}{$\mathrm{HCN}$} & $\mathrm{H}$ & 29.2 & 29.0 & 29.4 & 29.4 & 29.5 \\
\hline & $\mathrm{C}$ & 89.5 & 84.6 & 85.5 & 97.1 & 93.7 \\
\hline & $\mathrm{N}$ & -11.0 & -14.1 & -15.8 & 9.4 & 0.5 \\
\hline \multirow[t]{3}{*}{$\mathrm{HOF}$} & $\mathrm{O}$ & -49.0 & -68.9 & -46.1 & 20.2 & -24.8 \\
\hline & $\mathrm{H}$ & 20.6 & 19.6 & 21.2 & 22.8 & 21.7 \\
\hline & $\mathrm{F}$ & 184.9 & 192.2 & 210.2 & 210.7 & 185.5 \\
\hline \multirow[t]{2}{*}{$\mathrm{NH}_{3}$} & $\mathrm{~N}$ & 275.5 & 270.7 & 272.1 & 272.4 & 270.5 \\
\hline & $\mathrm{H}$ & 32.0 & 31.4 & 32.1 & 32.2 & 32.1 \\
\hline \multirow[t]{3}{*}{$\mathrm{CH}_{2} \mathrm{O}$} & $\mathrm{O}$ & -383.6 & -378.6 & -359.7 & -272.4 & -340.4 \\
\hline & $\mathrm{C}$ & 10.1 & 1.5 & 9.2 & 29.7 & 22.2 \\
\hline & $\mathrm{H}$ & 22.3 & 22.0 & 22.8 & 23.2 & 22.9 \\
\hline \multirow[t]{2}{*}{$\mathrm{CH}_{4}$} & $\mathrm{C}$ & 200.3 & 198.9 & 197.5 & 198.6 & 197.6 \\
\hline & $\mathrm{H}$ & 31.5 & 31.3 & 31.6 & 31.6 & 31.6 \\
\hline \multirow[t]{2}{*}{$\mathrm{C} 2 \mathrm{H} 4$} & $\mathrm{C}$ & 74.4 & 69.7 & 70.5 & 80.9 & 78.7 \\
\hline & $\mathrm{H}$ & 26.3 & 26.0 & 26.5 & 26.7 & 26.7 \\
\hline \multirow[t]{2}{*}{$\mathrm{AlF}$} & $\mathrm{Al}$ & 573.6 & 572.9 & 578.4 & 582.4 & 578.8 \\
\hline & $\mathrm{F}$ & 197.8 & 211.8 & 215.8 & 214.6 & 210.7 \\
\hline \multirow[t]{3}{*}{$\mathrm{CH}_{3} \mathrm{~F}$} & $\mathrm{C}$ & 125.8 & 122.1 & 125.8 & 129.1 & 127.8 \\
\hline & $\mathrm{F}$ & 480.3 & 482.9 & 478.5 & 473.0 & 475.7 \\
\hline & $\mathrm{H}$ & 27.6 & 27.3 & 28.0 & 28.0 & 28.0 \\
\hline $\mathrm{C}_{3} \mathrm{H}_{4}$ & $\mathrm{C}$ & 193.8 & 192.1 & 192.1 & 193.4 & 193.3 \\
\hline & $\mathrm{C}$ & 87.7 & 83.7 & 84.5 & 94.3 & 92.0 \\
\hline & $\mathrm{H}$ & 24.6 & 24.4 & 24.8 & 25.1 & 25.0 \\
\hline & $\mathrm{H}$ & 30.9 & 30.6 & 31.1 & 31.0 & 31.1 \\
\hline $\mathrm{FCCH}$ & $\mathrm{C}$ & 182.1 & 179.9 & 179.9 & 183.4 & 182.5 \\
\hline & $\mathrm{C}$ & 103.6 & 100.1 & 103.3 & 107.3 & 106.7 \\
\hline & $\mathrm{H}$ & 30.7 & 30.5 & 30.8 & 30.9 & 30.9 \\
\hline & $\mathrm{F}$ & 422.0 & 423.5 & 423.7 & 421.8 & 421.4 \\
\hline $\mathrm{FCN}$ & $\mathrm{F}$ & 373.2 & 374.1 & 377.3 & 375.9 & 371.4 \\
\hline & $\mathrm{C}$ & 86.6 & 82.2 & 83.8 & 91.4 & 89.8 \\
\hline & $\mathrm{N}$ & 120.3 & 117.9 & 114.5 & 130.1 & 124.5 \\
\hline $\mathrm{H}_{2} \mathrm{~S}$ & $\mathrm{~S}$ & 739.0 & 739.0 & 725.1 & 734.4 & 722.5 \\
\hline & $\mathrm{H}$ & 30.8 & 30.5 & 30.9 & 31.1 & 31.2 \\
\hline HCP & $\mathrm{H}$ & 29.6 & 29.6 & 30.0 & 29.8 & 30.0 \\
\hline & $\mathrm{C}$ & 42.3 & 37.6 & 35.0 & 51.8 & 50.3 \\
\hline & $\mathrm{P}$ & 393.6 & 388.0 & 376.2 & 426.9 & 417.7 \\
\hline $\mathrm{HFCO}$ & $\mathrm{O}$ & -92.3 & -94.3 & -87.8 & -58.6 & -81.3 \\
\hline & $\mathrm{C}$ & 45.0 & 39.6 & 43.1 & 52.1 & 48.7 \\
\hline & $\mathrm{F}$ & 166.3 & 165.3 & 182.9 & 183.2 & 172.3 \\
\hline & $\mathrm{H}$ & 24.3 & 23.9 & 24.6 & 24.6 & 24.5 \\
\hline $\mathrm{H}_{2} \mathrm{C}_{2} \mathrm{O}$ & $\mathrm{C}$ & 195.0 & 193.3 & 192.8 & 194.3 & 192.9 \\
\hline & $\mathrm{C}$ & 0.4 & -6.3 & -1.2 & 9.4 & 2.7 \\
\hline & $\mathrm{O}$ & -6.9 & -5.9 & 0.6 & 15.0 & -1.0 \\
\hline & $\mathrm{H}$ & 29.5 & 29.2 & 29.7 & 29.8 & 29.7 \\
\hline $\mathrm{LiF}$ & $\mathrm{Li}$ & 90.3 & 89.3 & 90.8 & 91.0 & 91.5 \\
\hline & $\mathrm{F}$ & 364.2 & 382.5 & 373.2 & 373.7 & 377.6 \\
\hline $\mathrm{LiH}$ & $\mathrm{H}$ & 26.5 & 26.6 & 26.8 & 26.5 & 26.7 \\
\hline & $\mathrm{Li}$ & 89.9 & 89.3 & 90.1 & 91.3 & 91.6 \\
\hline $\mathrm{N}_{2} \mathrm{O}$ & $\mathrm{N}$ & 111.1 & 106.4 & 103.7 & 122.6 & 114.7 \\
\hline & $\mathrm{N}$ & 19.0 & 12.6 & 9.9 & 31.5 & 26.2 \\
\hline & $\mathrm{O}$ & 196.6 & 199.0 & 196.0 & 206.1 & 197.7 \\
\hline OCS & $\mathrm{O}$ & 96.6 & 96.8 & 97.8 & 110.3 & 105.0 \\
\hline & $\mathrm{C}$ & 35.4 & 30.2 & 29.4 & 44.9 & 42.2 \\
\hline & $\mathrm{S}$ & 800.2 & 796.7 & 800.5 & 810.1 & 799.2 \\
\hline $\mathrm{OF}_{2}$ & $\mathrm{O}$ & -412.4 & -447.1 & -387.7 & -239.0 & -352.7 \\
\hline & $\mathrm{F}$ & -6.2 & -24.0 & 21.2 & 43.1 & 4.0 \\
\hline $\mathrm{H}_{4} \mathrm{C}_{2} \mathrm{O}$ & $\mathrm{O}$ & 362.4 & 363.2 & 365.1 & 359.2 & 359.4 \\
\hline & $\mathrm{C}$ & 156.3 & 153.2 & 155.9 & 158.0 & 157.0 \\
\hline & $\mathrm{H}$ & 29.4 & 29.1 & 29.7 & 29.6 & 29.7 \\
\hline $\mathrm{PN}$ & $\mathrm{N}$ & -342.9 & -344.0 & -359.2 & -273.0 & -304.1 \\
\hline & $\mathrm{P}$ & 59.8 & 50.6 & 27.1 & 151.2 & 105.8 \\
\hline
\end{tabular}


Table 22: Statistics for absolute isotropic NMR shieldings in a cc-pCVTZ basis. CCSD(T)/aug-cc$\mathrm{pCV}[\mathrm{TQ}] \mathrm{Z}$ shieldings are used as a reference. $\mathrm{MSD}=$ mean signed deviation; $\mathrm{MAD}=$ mean absolute deviation; $\mathrm{MRD}=$ mean relative deviation [\%]; $\mathrm{MARD}=$ mean absolute relative deviation [\%]; $\mathrm{RMSD}=$ root mean squared deviation.

\begin{tabular}{lrrrrr}
\hline \hline & MSD & MAD & MRD & MARD & RMSD \\
\hline RHF & -10.8 & 18.7 & -46.0 & 57.1 & 36.9 \\
CCSD & 1.5 & 4.5 & 9.7 & 15.2 & 9.7 \\
CCSD(T) & 2.3 & 3.9 & 18.2 & 19.3 & 6.9 \\
RPA@HF & 2.4 & 5.4 & 13.7 & 17.1 & 11.3 \\
RPA@PBE & 16.5 & 17.4 & 74.2 & 74.5 & 36.9 \\
$\sigma-\mathrm{P} 1 @ P B E$ & 7.9 & 9.5 & 45.2 & 45.6 & 17.9 \\
\hline \hline
\end{tabular}

Table 23: Statistics for absolute isotropic NMR shieldings in a cc-pCVTZ basis. CCSD(T) shieldings are used as a reference. $\mathrm{MSD}=$ mean signed deviation; $\mathrm{MAD}=$ mean absolute deviation; $\mathrm{MRD}=$ mean relative deviation [\%]; MARD=mean absolute relative deviation [\%]; RMSD=root mean squared deviation.

\begin{tabular}{lrrrrr}
\hline \hline & MSD & MAD & MRD & MARD & RMSD \\
\hline RHF & -13.1 & 20.3 & -66.0 & 90.4 & 38.6 \\
CCSD & -0.8 & 3.5 & -7.4 & 14.9 & 6.6 \\
CCSD(T) & 0.0 & 0.0 & 0.0 & 0.0 & 0.0 \\
RPA@HF & 0.1 & 5.2 & -1.4 & 20.0 & 9.1 \\
RPA@PBE & 14.2 & 15.0 & 70.1 & 70.3 & 32.3 \\
$\sigma$-P1@PBE & 5.6 & 7.0 & 22.4 & 22.8 & 13.4 \\
\hline \hline
\end{tabular}


Table 24: Absolute isotropic NMR shieldings in a cc-pCVQZ basis. All values are given in ppm. "Extrap." stands for basis-set extrapolated CCSD(T)-shieldings in an aug-cc-pCV[TQ]Z basis set. The data for "Extrap." was taken from Ref. 3. CCSD(T) calculations were done with the CFOUR program[1, [2].

\begin{tabular}{|c|c|c|c|c|c|c|}
\hline Mol. & Nuc. & $\operatorname{CCSD}(\mathrm{T})$ & Extrap. & $\overline{\mathrm{RPA} @ \mathrm{HF}}$ & RPA@PBE & $\sigma-\mathrm{P} 1 @ \mathrm{PBE}$ \\
\hline \multirow[t]{2}{*}{$\mathrm{HF}$} & $\mathrm{H}$ & 29.1 & 28.8 & 29.0 & 29.6 & 29.1 \\
\hline & $\mathrm{F}$ & 419.7 & 420.3 & 419.0 & 418.3 & 418.0 \\
\hline \multirow[t]{2}{*}{$\mathrm{CO}$} & $\mathrm{C}$ & 3.9 & 2.2 & -3.0 & 20.6 & 9.7 \\
\hline & $\mathrm{O}$ & -54.9 & -55.0 & -54.5 & -27.9 & -41.7 \\
\hline $\mathrm{N}_{2}$ & $\mathrm{~N}$ & -60.2 & -60.4 & -67.7 & -37.2 & -52.0 \\
\hline \multirow[t]{2}{*}{$\mathrm{H}_{2} \mathrm{O}$} & $\mathrm{O}$ & 340.6 & 338.0 & 338.0 & 338.0 & 337.3 \\
\hline & $\mathrm{H}$ & 30.9 & 30.6 & 30.9 & 31.2 & 30.9 \\
\hline \multirow[t]{3}{*}{$\mathrm{HCN}$} & $\mathrm{H}$ & 29.1 & 29.0 & 29.3 & 29.3 & 29.2 \\
\hline & $\mathrm{C}$ & 86.0 & 84.6 & 81.7 & 93.1 & 88.9 \\
\hline & $\mathrm{N}$ & -13.6 & -14.1 & -18.7 & 6.1 & -3.3 \\
\hline \multirow{3}{*}{$\mathrm{HOF}$} & $\mathrm{O}$ & -61.8 & -68.9 & -61.6 & 5.9 & -39.7 \\
\hline & $\mathrm{H}$ & 20.0 & 19.6 & 20.5 & 22.0 & 20.6 \\
\hline & $\mathrm{F}$ & 189.1 & 192.2 & 213.4 & 211.6 & 191.9 \\
\hline \multirow[t]{2}{*}{$\mathrm{NH}_{3}$} & $\mathrm{~N}$ & 272.7 & 270.7 & 269.4 & 270.2 & 269.5 \\
\hline & $\mathrm{H}$ & 31.6 & 31.4 & 31.7 & 31.8 & 31.6 \\
\hline \multirow[t]{3}{*}{$\mathrm{CH}_{2} \mathrm{O}$} & $\mathrm{O}$ & -382.9 & -378.6 & -357.5 & -275.8 & -336.7 \\
\hline & $\mathrm{C}$ & 4.3 & 1.5 & 3.1 & 22.8 & 13.5 \\
\hline & $\mathrm{H}$ & 22.1 & 22.0 & 22.6 & 23.0 & 22.6 \\
\hline \multirow[t]{2}{*}{$\mathrm{CH}_{4}$} & $\mathrm{C}$ & 199.2 & 198.9 & 196.3 & 197.6 & 197.3 \\
\hline & $\mathrm{H}$ & 31.4 & 31.3 & 31.5 & 31.4 & 31.4 \\
\hline \multirow[t]{2}{*}{$\mathrm{C} 2 \mathrm{H} 4$} & $\mathrm{C}$ & 71.0 & 69.7 & 66.9 & 77.1 & 74.1 \\
\hline & $\mathrm{H}$ & 26.1 & 26.0 & 26.3 & 26.5 & 26.4 \\
\hline \multirow[t]{2}{*}{$\mathrm{AlF}$} & $\mathrm{Al}$ & 573.2 & 572.9 & 576.7 & 579.8 & 576.2 \\
\hline & $\mathrm{F}$ & 206.8 & 211.8 & 223.6 & 223.5 & 225.1 \\
\hline \multirow[t]{3}{*}{$\mathrm{CH}_{3} \mathrm{~F}$} & $\mathrm{C}$ & 123.2 & 122.1 & 123.3 & 126.5 & 125.1 \\
\hline & $\mathrm{F}$ & 482.4 & 482.9 & 479.7 & 475.2 & 480.3 \\
\hline & $\mathrm{H}$ & 27.4 & 27.3 & 27.8 & 27.7 & 27.7 \\
\hline $\mathrm{C}_{3} \mathrm{H}_{4}$ & $\mathrm{C}$ & 192.5 & 192.1 & 190.6 & 191.9 & 191.8 \\
\hline & $\mathrm{C}$ & 84.8 & 83.7 & 81.2 & 90.9 & 88.2 \\
\hline & $\mathrm{H}$ & 24.4 & 24.4 & 24.6 & 24.9 & 24.7 \\
\hline & $\mathrm{H}$ & 30.7 & 30.6 & 30.9 & 30.9 & 30.8 \\
\hline $\mathrm{FCCH}$ & $\mathrm{C}$ & 180.5 & 179.9 & 178.1 & 181.3 & 179.8 \\
\hline & $\mathrm{C}$ & 101.0 & 100.1 & 100.7 & 104.7 & 103.8 \\
\hline & $\mathrm{H}$ & 30.5 & 30.5 & 30.6 & 30.8 & 30.7 \\
\hline & $\mathrm{F}$ & 422.6 & 423.5 & 423.7 & 422.3 & 423.9 \\
\hline $\mathrm{FCN}$ & $\mathrm{F}$ & 373.6 & 374.1 & 377.1 & 376.0 & 374.4 \\
\hline & $\mathrm{C}$ & 83.4 & 82.2 & 80.5 & 87.9 & 85.6 \\
\hline & $\mathrm{N}$ & 118.4 & 117.9 & 112.1 & 127.3 & 120.8 \\
\hline $\mathrm{H}_{2} \mathrm{~S}$ & S & 737.9 & 739.0 & 722.8 & 733.1 & 725.5 \\
\hline & $\mathrm{H}$ & 30.6 & 30.5 & 30.7 & 30.8 & 30.7 \\
\hline $\mathrm{HCP}$ & $\mathrm{H}$ & 29.6 & 29.6 & 29.9 & 29.8 & 29.8 \\
\hline & $\mathrm{C}$ & 38.8 & 37.6 & 31.1 & 47.6 & 44.4 \\
\hline & $\mathrm{P}$ & 390.5 & 388.0 & 373.0 & 421.9 & 409.0 \\
\hline $\mathrm{HFCO}$ & $\mathrm{O}$ & -94.7 & -94.3 & -90.2 & -62.5 & -82.4 \\
\hline & $\mathrm{C}$ & 41.2 & 39.6 & 38.9 & 47.6 & 43.4 \\
\hline & $\mathrm{F}$ & 164.9 & 165.3 & 181.4 & 182.2 & 175.3 \\
\hline & $\mathrm{H}$ & 24.0 & 23.9 & 24.3 & 24.3 & 24.2 \\
\hline $\mathrm{H}_{2} \mathrm{C}_{2} \mathrm{O}$ & $\mathrm{C}$ & 193.7 & 193.3 & 191.2 & 192.8 & 191.6 \\
\hline & $\mathrm{C}$ & -4.4 & -6.3 & -6.6 & 4.0 & -2.9 \\
\hline & $\mathrm{O}$ & -7.2 & -5.9 & -0.3 & 13.7 & 1.7 \\
\hline & $\mathrm{H}$ & 29.3 & 29.2 & 29.5 & 29.5 & 29.3 \\
\hline $\mathrm{LiF}$ & $\mathrm{Li}$ & 89.6 & 89.3 & 90.1 & 90.3 & 90.9 \\
\hline & $\mathrm{F}$ & 375.0 & 382.5 & 383.3 & 385.0 & 392.4 \\
\hline $\mathrm{LiH}$ & $\mathrm{H}$ & 26.6 & 26.6 & 26.9 & 26.6 & 26.8 \\
\hline & $\mathrm{Li}$ & 89.5 & 89.3 & 89.7 & 90.7 & 90.8 \\
\hline $\mathrm{N}_{2} \mathrm{O}$ & $\mathrm{N}$ & 107.9 & 106.4 & 99.8 & 118.7 & 110.8 \\
\hline & $\mathrm{N}$ & 14.0 & 12.6 & 4.7 & 26.1 & 21.1 \\
\hline & $\mathrm{O}$ & 197.5 & 199.0 & 195.9 & 206.0 & 199.0 \\
\hline OCS & $\mathrm{O}$ & 96.2 & 96.8 & 97.1 & 109.2 & 106.1 \\
\hline & $\mathrm{C}$ & 31.5 & 30.2 & 25.2 & 40.5 & 37.4 \\
\hline & $\mathrm{S}$ & 798.5 & 796.7 & 797.4 & 806.0 & 795.7 \\
\hline $\mathrm{OF}_{2}$ & $\mathrm{O}$ & -435.0 & -447.1 & -411.6 & -265.2 & -378.0 \\
\hline & $\mathrm{F}$ & -15.7 & -24.0 & 10.7 & 31.5 & -3.7 \\
\hline $\mathrm{H}_{4} \mathrm{C}_{2} \mathrm{O}$ & $\mathrm{O}$ & 363.3 & 363.2 & 364.9 & 359.2 & 359.8 \\
\hline & $\mathrm{C}$ & 154.0 & 153.2 & 153.4 & 155.6 & 154.3 \\
\hline & $\mathrm{H}$ & 29.2 & 29.1 & 29.5 & 29.4 & 29.3 \\
\hline $\mathrm{PN}$ & $\mathrm{N}$ & -345.5 & -344.0 & -362.3 & -279.8 & -309.9 \\
\hline & $\mathrm{P}$ & 55.3 & 50.6 & 21.7 & 140.9 & 96.2 \\
\hline
\end{tabular}


Table 25: Statistics for absolute isotropic NMR shieldings in a cc-pCVQZ basis. CCSD(T)/aug-cc$\mathrm{pCV}[\mathrm{TQ}] \mathrm{Z}$ shieldings are used as a reference. $\mathrm{MSD}=$ mean signed deviation; $\mathrm{MAD}=$ mean absolute deviation; $\mathrm{MRD}=$ mean relative deviation [\%]; $\mathrm{MARD}=$ mean absolute relative deviation [\%]; $\mathrm{RMSD}=$ root mean squared deviation.

\begin{tabular}{lrrrrr}
\hline \hline & MSD & MAD & MRD & MARD & RMSD \\
\hline RHF & -12.1 & 19.2 & -55.1 & 64.7 & 37.2 \\
CCSD & -0.2 & 3.7 & -3.1 & 10.2 & 7.4 \\
CCSD(T) & 0.6 & 1.4 & 5.4 & 6.4 & 2.6 \\
RPA@HF & 0.4 & 4.9 & -0.8 & 13.4 & 9.3 \\
RPA@PBE & 14.1 & 14.8 & 58.3 & 58.5 & 32.5 \\
$\sigma-\mathrm{P} 1 @ P B E$ & 6.2 & 7.0 & 28.0 & 28.2 & 14.0 \\
\hline \hline
\end{tabular}

Table 26: Statistics for absolute isotropic NMR shieldings in a cc-pCVQZ basis. CCSD(T) shieldings are used as a reference. $\mathrm{MSD}=$ mean signed deviation; $\mathrm{MAD}=$ mean absolute deviation; $\mathrm{MRD}=$ mean relative deviation [\%]; MARD=mean absolute relative deviation [\%]; RMSD=root mean squared deviation.

\begin{tabular}{lrrrrr}
\hline \hline & MSD & MAD & MRD & MARD & RMSD \\
\hline RHF & -12.7 & 19.7 & -41.9 & 53.3 & 37.6 \\
CCSD & -0.8 & 3.5 & -5.4 & 9.1 & 6.6 \\
CCSD(T) & 0.0 & 0.0 & 0.0 & 0.0 & 0.0 \\
RPA@HF & -0.2 & 5.2 & -2.9 & 12.7 & 9.1 \\
RPA@PBE & 13.5 & 14.3 & 37.5 & 37.7 & 31.1 \\
$\sigma$-P1@PBE & 5.6 & 6.6 & 15.1 & 15.4 & 12.9 \\
\hline \hline
\end{tabular}


Table 27: Absolute isotropic NMR shieldings in a def2-SVP basis. All values are given in ppm. "Extrap." stands for basis-set extrapolated $\operatorname{CCSD}(\mathrm{T})$-shieldings in an aug-cc-pCV[TQ]Z basis set. The data for "Extrap." was taken from Ref. 3. CCSD(T) calculations were done with the CFOUR program[1, 2.

\begin{tabular}{|c|c|c|c|c|c|}
\hline Mol. & Nuc. & $\overline{C C C S D(T)}$ & Extrap. & RPA@HF & RPA@PBE \\
\hline \multirow[t]{2}{*}{$\mathrm{HF}$} & $\mathrm{H}$ & 30.1 & 28.8 & 30.0 & 30.6 \\
\hline & $\mathrm{F}$ & 430.6 & 420.3 & 430.7 & 428.6 \\
\hline \multirow[t]{2}{*}{$\mathrm{CO}$} & $\mathrm{C}$ & 32.2 & 2.2 & 28.8 & 52.7 \\
\hline & $\mathrm{O}$ & -10.5 & -55.0 & -5.4 & 23.6 \\
\hline $\mathrm{N}_{2}$ & $\mathrm{~N}$ & -20.8 & -60.4 & -22.0 & 7.0 \\
\hline \multirow[t]{2}{*}{$\mathrm{H}_{2} \mathrm{O}$} & $\mathrm{O}$ & 355.2 & 338.0 & 353.9 & 352.0 \\
\hline & $\mathrm{H}$ & 31.7 & 30.6 & 31.7 & 32.1 \\
\hline \multirow[t]{3}{*}{$\mathrm{HCN}$} & $\mathrm{H}$ & 29.8 & 29.0 & 29.9 & 30.0 \\
\hline & $\mathrm{C}$ & 108.4 & 84.6 & 105.2 & 116.7 \\
\hline & $\mathrm{N}$ & 17.9 & -14.1 & 16.1 & 41.7 \\
\hline \multirow[t]{3}{*}{$\mathrm{HOF}$} & $\mathrm{O}$ & -4.6 & -68.9 & 6.9 & 73.4 \\
\hline & $\mathrm{H}$ & 21.7 & 19.6 & 22.4 & 24.2 \\
\hline & $\mathrm{F}$ & 193.0 & 192.2 & 217.0 & 231.8 \\
\hline \multirow[t]{2}{*}{$\mathrm{NH}_{3}$} & $\mathrm{~N}$ & 280.7 & 270.7 & 278.4 & 278.0 \\
\hline & $\mathrm{H}$ & 32.3 & 31.4 & 32.4 & 32.5 \\
\hline \multirow[t]{3}{*}{$\mathrm{CH}_{2} \mathrm{O}$} & $\mathrm{O}$ & -338.7 & -378.6 & -307.9 & -202.0 \\
\hline & $\mathrm{C}$ & 33.3 & 1.5 & 32.6 & 54.7 \\
\hline & $\mathrm{H}$ & 23.1 & 22.0 & 23.5 & 24.2 \\
\hline \multirow[t]{2}{*}{$\mathrm{CH}_{4}$} & $\mathrm{C}$ & 205.5 & 198.9 & 203.3 & 204.2 \\
\hline & $\mathrm{H}$ & 31.6 & 31.3 & 31.7 & 31.7 \\
\hline \multirow{2}{*}{$\mathrm{C} 2 \mathrm{H} 4$} & C & 92.3 & 69.7 & 88.1 & 99.1 \\
\hline & $\mathrm{H}$ & 26.8 & 26.0 & 26.9 & 27.2 \\
\hline \multirow[t]{2}{*}{$\mathrm{AlF}$} & $\mathrm{Al}$ & 583.4 & 572.9 & 587.7 & 592.2 \\
\hline & $\mathrm{F}$ & 205.1 & 211.8 & 223.9 & 224.6 \\
\hline \multirow[t]{3}{*}{$\mathrm{CH}_{3} \mathrm{~F}$} & $\mathrm{C}$ & 138.4 & 122.1 & 138.2 & 141.9 \\
\hline & $\mathrm{F}$ & 481.2 & 482.9 & 481.3 & 475.4 \\
\hline & $\mathrm{H}$ & 28.0 & 27.3 & 28.3 & 28.4 \\
\hline \multirow[t]{4}{*}{$\mathrm{C}_{3} \mathrm{H}_{4}$} & $\mathrm{C}$ & 202.9 & 192.1 & 201.6 & 203.2 \\
\hline & $\mathrm{C}$ & 104.8 & 83.7 & 102.3 & 112.1 \\
\hline & $\mathrm{H}$ & 25.2 & 24.4 & 25.4 & 25.8 \\
\hline & $\mathrm{H}$ & 31.3 & 30.6 & 31.5 & 31.5 \\
\hline $\mathrm{FCCH}$ & $\mathrm{C}$ & 188.9 & 179.9 & 186.6 & 190.3 \\
\hline & $\mathrm{C}$ & 121.7 & 100.1 & 121.0 & 125.2 \\
\hline & $\mathrm{H}$ & 31.2 & 30.5 & 31.4 & 31.5 \\
\hline & $\mathrm{F}$ & 438.9 & 423.5 & 441.2 & 441.2 \\
\hline $\mathrm{FCN}$ & $\mathrm{F}$ & 398.3 & 374.1 & 403.8 & 404.5 \\
\hline & C & 105.3 & 82.2 & 103.0 & 110.2 \\
\hline & $\mathrm{N}$ & 134.1 & 117.9 & 130.3 & 146.4 \\
\hline $\mathrm{H}_{2} \mathrm{~S}$ & $\mathrm{~S}$ & 786.6 & 739.0 & 780.0 & 787.0 \\
\hline & $\mathrm{H}$ & 31.5 & 30.5 & 31.6 & 31.8 \\
\hline $\mathrm{HCP}$ & $\mathrm{H}$ & 29.8 & 29.6 & 30.1 & 30.0 \\
\hline & C & 63.0 & 37.6 & 55.9 & 72.8 \\
\hline & $\mathrm{P}$ & 462.9 & 388.0 & 445.7 & 500.7 \\
\hline $\mathrm{HFCO}$ & $\mathrm{O}$ & -58.8 & -94.3 & -49.6 & -16.2 \\
\hline & $\mathrm{C}$ & 64.6 & 39.6 & 62.9 & 72.3 \\
\hline & $\mathrm{F}$ & 196.8 & 165.3 & 212.8 & 217.1 \\
\hline & $\mathrm{H}$ & 25.1 & 23.9 & 25.4 & 25.5 \\
\hline $\mathrm{H}_{2} \mathrm{C}_{2} \mathrm{O}$ & $\mathrm{C}$ & 203.9 & 193.3 & 202.7 & 204.2 \\
\hline & $\mathrm{C}$ & 21.3 & -6.3 & 21.4 & 31.8 \\
\hline & $\mathrm{O}$ & 21.3 & -5.9 & 36.4 & 51.1 \\
\hline & $\mathrm{H}$ & 30.1 & 29.2 & 30.3 & 30.4 \\
\hline $\mathrm{LiF}$ & $\mathrm{Li}$ & 89.3 & 89.3 & 89.7 & 89.6 \\
\hline & $\mathrm{F}$ & 338.0 & 382.5 & 349.7 & 338.9 \\
\hline $\mathrm{LiH}$ & $\mathrm{H}$ & 26.7 & 26.6 & 26.9 & 26.6 \\
\hline & $\mathrm{Li}$ & 90.3 & 89.3 & 90.4 & 92.4 \\
\hline $\mathrm{N}_{2} \mathrm{O}$ & $\mathrm{N}$ & 133.5 & 106.4 & 131.0 & 147.5 \\
\hline & $\mathrm{N}$ & 44.5 & 12.6 & 39.8 & 58.8 \\
\hline & $\mathrm{O}$ & 224.7 & 199.0 & 229.9 & 237.2 \\
\hline OCS & $\mathrm{O}$ & 131.8 & 96.8 & 135.9 & 148.9 \\
\hline & $\mathrm{C}$ & 54.7 & 30.2 & 50.4 & 65.0 \\
\hline & $\mathrm{S}$ & 849.1 & 796.7 & 852.3 & 865.3 \\
\hline $\mathrm{OF}_{2}$ & $\mathrm{O}$ & -329.6 & -447.1 & -300.0 & -132.4 \\
\hline & $\mathrm{F}$ & 27.3 & -24.0 & 58.0 & 88.7 \\
\hline $\mathrm{H}_{4} \mathrm{C}_{2} \mathrm{O}$ & $\mathrm{O}$ & 368.4 & 363.2 & 372.4 & 367.4 \\
\hline & $\mathrm{C}$ & 166.9 & 153.2 & 166.6 & 169.1 \\
\hline & $\mathrm{H}$ & 29.8 & 29.1 & 30.1 & 30.1 \\
\hline $\mathrm{PN}$ & $\mathrm{N}$ & -282.6 & -344.0 & -278.6 & -194.6 \\
\hline & $\mathrm{P}$ & 183.8 & 50.6 & 167.1 & 292.3 \\
\hline
\end{tabular}


Table 28: Statistics for absolute isotropic NMR shieldings in a def2-SVP basis. CCSD(T)/aug-cc$\mathrm{pCV}[\mathrm{TQ}] \mathrm{Z}$ shieldings are used as a reference. $\mathrm{MSD}=$ mean signed deviation; $\mathrm{MAD}=$ mean absolute deviation; $\mathrm{MRD}=$ mean relative deviation [\%]; $\mathrm{MARD}=$ mean absolute relative deviation [\%]; $\mathrm{RMSD}=$ root mean squared deviation.

\begin{tabular}{lrrrrr}
\hline \hline & MSD & MAD & MRD & MARD & RMSD \\
\hline CCSD(T) & 20.4 & 22.0 & 92.2 & 92.7 & 33.7 \\
RPA@HF & 22.5 & 23.6 & 94.3 & 94.6 & 36.4 \\
RPA@PBE & 37.5 & 39.0 & 157.1 & 157.5 & 68.5 \\
\hline \hline
\end{tabular}

Table 29: Statistics for absolute isotropic NMR shieldings in a def2-SVP basis. CCSD(T) shieldings are used as a reference. $\mathrm{MSD}=$ mean signed deviation; $\mathrm{MAD}=$ mean absolute deviation; $\mathrm{MRD}=$ mean relative deviation [\%]; MARD=mean absolute relative deviation [\%]; RMSD=root mean squared deviation.

\begin{tabular}{lrrrrr}
\hline \hline & MSD & MAD & MRD & MARD & RMSD \\
\hline CCSD(T) & 0.0 & 0.0 & 0.0 & 0.0 & 0.0 \\
RPA@HF & 2.1 & 4.9 & 7.0 & 9.9 & 9.0 \\
RPA@PBE & 17.1 & 17.6 & 48.9 & 49.1 & 38.1 \\
\hline \hline
\end{tabular}


Table 30: Absolute isotropic NMR shieldings in a def2-TZVP basis. All values are given in ppm. "Extrap." stands for basis-set extrapolated CCSD(T)-shieldings in an aug-cc-pCV[TQ]Z basis set. The data for "Extrap." was taken from Ref. 3. CCSD(T) calculations were done with the CFOUR program[1, [2].

\begin{tabular}{|c|c|c|c|c|c|}
\hline Mol. & Nuc. & $\operatorname{CCSD}(\mathrm{T})$ & Extrap. & RPA@HF & RPA@PBE \\
\hline \multirow[t]{2}{*}{$\mathrm{HF}$} & $\mathrm{H}$ & 29.7 & 28.8 & 29.6 & 30.2 \\
\hline & $\mathrm{F}$ & 420.8 & 420.3 & 420.7 & 420.1 \\
\hline \multirow[t]{2}{*}{$\mathrm{CO}$} & $\mathrm{C}$ & 10.4 & 2.2 & 4.8 & 27.7 \\
\hline & $\mathrm{O}$ & -50.9 & -55.0 & -49.2 & -21.4 \\
\hline $\mathrm{N}_{2}$ & $\mathrm{~N}$ & -52.2 & -60.4 & -58.1 & -28.4 \\
\hline \multirow[t]{2}{*}{$\mathrm{H}_{2} \mathrm{O}$} & $\mathrm{O}$ & 343.3 & 338.0 & 341.7 & 341.9 \\
\hline & $\mathrm{H}$ & 31.6 & 30.6 & 31.6 & 31.9 \\
\hline \multirow[t]{3}{*}{$\mathrm{HCN}$} & $\mathrm{H}$ & 29.3 & 29.0 & 29.4 & 29.5 \\
\hline & $\mathrm{C}$ & 90.3 & 84.6 & 86.2 & 97.0 \\
\hline & $\mathrm{N}$ & -8.3 & -14.1 & -12.8 & 12.8 \\
\hline \multirow[t]{3}{*}{$\mathrm{HOF}$} & $\mathrm{O}$ & -53.7 & -68.9 & -50.1 & 17.1 \\
\hline & $\mathrm{H}$ & 20.9 & 19.6 & 21.4 & 23.0 \\
\hline & $\mathrm{F}$ & 188.0 & 192.2 & 213.4 & 213.4 \\
\hline \multirow[t]{2}{*}{$\mathrm{NH}_{3}$} & $\mathrm{~N}$ & 275.8 & 270.7 & 273.3 & 274.2 \\
\hline & $\mathrm{H}$ & 32.2 & 31.4 & 32.3 & 32.4 \\
\hline \multirow[t]{3}{*}{$\mathrm{CH}_{2} \mathrm{O}$} & $\mathrm{O}$ & -374.8 & -378.6 & -350.4 & -266.7 \\
\hline & C & 10.7 & 1.5 & 9.3 & 28.2 \\
\hline & $\mathrm{H}$ & 22.4 & 22.0 & 22.9 & 23.3 \\
\hline \multirow[t]{2}{*}{$\mathrm{CH}_{4}$} & $\mathrm{C}$ & 201.1 & 198.9 & 198.7 & 200.3 \\
\hline & $\mathrm{H}$ & 31.7 & 31.3 & 31.8 & 31.8 \\
\hline \multirow[t]{2}{*}{$\mathrm{C} 2 \mathrm{H} 4$} & $\mathrm{C}$ & 75.8 & 69.7 & 71.6 & 81.5 \\
\hline & $\mathrm{H}$ & 26.5 & 26.0 & 26.6 & 26.8 \\
\hline \multirow{2}{*}{$\mathrm{AlF}$} & $\mathrm{Al}$ & 574.6 & 572.9 & 579.9 & 584.5 \\
\hline & $\mathrm{F}$ & 203.3 & 211.8 & 220.7 & 222.0 \\
\hline \multirow[t]{3}{*}{$\mathrm{CH}_{3} \mathrm{~F}$} & C & 126.5 & 122.1 & 126.6 & 129.8 \\
\hline & $\mathrm{F}$ & 482.1 & 482.9 & 480.6 & 476.0 \\
\hline & $\mathrm{H}$ & 27.8 & 27.3 & 28.1 & 28.1 \\
\hline \multirow{4}{*}{$\mathrm{C}_{3} \mathrm{H}_{4}$} & C & 195.3 & 192.1 & 193.8 & 195.6 \\
\hline & $\mathrm{C}$ & 89.4 & 83.7 & 86.0 & 96.0 \\
\hline & $\mathrm{H}$ & 24.8 & 24.4 & 24.9 & 25.2 \\
\hline & $\mathrm{H}$ & 31.0 & 30.6 & 31.2 & 31.2 \\
\hline $\mathrm{FCCH}$ & $\mathrm{C}$ & 184.5 & 179.9 & 182.2 & 185.4 \\
\hline & $\mathrm{C}$ & 104.5 & 100.1 & 103.9 & 107.8 \\
\hline & $\mathrm{H}$ & 30.9 & 30.5 & 31.0 & 31.1 \\
\hline & $\mathrm{F}$ & 426.0 & 423.5 & 427.4 & 427.9 \\
\hline $\mathrm{FCN}$ & $\mathrm{F}$ & 374.1 & 374.1 & 378.8 & 378.8 \\
\hline & C & 87.3 & 82.2 & 84.5 & 91.6 \\
\hline & $\mathrm{N}$ & 123.3 & 117.9 & 117.9 & 133.6 \\
\hline $\mathrm{H}_{2} \mathrm{~S}$ & $\mathrm{~S}$ & 729.2 & 739.0 & 718.4 & 729.4 \\
\hline & $\mathrm{H}$ & 31.0 & 30.5 & 31.2 & 31.3 \\
\hline HCP & $\mathrm{H}$ & 29.7 & 29.6 & 30.0 & 29.9 \\
\hline & $\mathrm{C}$ & 44.1 & 37.6 & 36.7 & 52.4 \\
\hline & $\mathrm{P}$ & 381.4 & 388.0 & 363.4 & 414.4 \\
\hline $\mathrm{HFCO}$ & $\mathrm{O}$ & -88.3 & -94.3 & -83.0 & -53.8 \\
\hline & C & 45.8 & 39.6 & 43.8 & 52.3 \\
\hline & $\mathrm{F}$ & 165.7 & 165.3 & 182.3 & 184.1 \\
\hline & $\mathrm{H}$ & 24.4 & 23.9 & 24.7 & 24.7 \\
\hline $\mathrm{H}_{2} \mathrm{C}_{2} \mathrm{O}$ & $\mathrm{C}$ & 197.1 & 193.3 & 195.3 & 197.0 \\
\hline & $\mathrm{C}$ & 1.8 & -6.3 & 0.4 & 10.9 \\
\hline & $\mathrm{O}$ & -2.6 & -5.9 & 6.5 & 22.3 \\
\hline & $\mathrm{H}$ & 29.8 & 29.2 & 29.9 & 30.0 \\
\hline $\mathrm{LiF}$ & $\mathrm{Li}$ & 90.6 & 89.3 & 91.0 & 91.3 \\
\hline & $\mathrm{F}$ & 378.0 & 382.5 & 386.8 & 392.1 \\
\hline $\mathrm{LiH}$ & $\mathrm{H}$ & 26.6 & 26.6 & 26.9 & 26.6 \\
\hline & $\mathrm{Li}$ & 91.1 & 89.3 & 91.2 & 93.2 \\
\hline $\mathrm{N}_{2} \mathrm{O}$ & $\mathrm{N}$ & 114.1 & 106.4 & 107.7 & 125.8 \\
\hline & $\mathrm{N}$ & 18.7 & 12.6 & 10.8 & 31.4 \\
\hline & $\mathrm{O}$ & 200.6 & 199.0 & 201.2 & 211.3 \\
\hline OCS & $\mathrm{O}$ & 100.4 & 96.8 & 102.6 & 115.4 \\
\hline & C & 36.4 & 30.2 & 31.0 & 45.7 \\
\hline & $\mathrm{S}$ & 797.7 & 796.7 & 799.4 & 808.0 \\
\hline $\mathrm{OF}_{2}$ & $\mathrm{O}$ & -419.7 & -447.1 & -393.0 & -242.5 \\
\hline & $\mathrm{F}$ & -12.8 & -24.0 & 15.5 & 37.6 \\
\hline $\mathrm{H}_{4} \mathrm{C}_{2} \mathrm{O}$ & $\mathrm{O}$ & 366.1 & 363.2 & 368.8 & 363.5 \\
\hline & $\mathrm{C}$ & 157.3 & 153.2 & 156.8 & 159.1 \\
\hline & $\mathrm{H}$ & 29.6 & 29.1 & 29.8 & 29.7 \\
\hline $\mathrm{PN}$ & $\mathrm{N}$ & -336.2 & -344.0 & -347.2 & -267.4 \\
\hline & $\mathrm{P}$ & 40.0 & 50.6 & 11.8 & 134.6 \\
\hline
\end{tabular}


Table 31: Statistics for absolute isotropic NMR shieldings in a def2-TZVP basis. CCSD(T)/aug-cc$\mathrm{pCV}[\mathrm{TQ}] \mathrm{Z}$ shieldings are used as a reference. $\mathrm{MSD}=$ mean signed deviation; $\mathrm{MAD}=$ mean absolute deviation; $\mathrm{MRD}=$ mean relative deviation [\%]; $\mathrm{MARD}=$ mean absolute relative deviation [\%]; $\mathrm{RMSD}=$ root mean squared deviation.

\begin{tabular}{lrrrrr}
\hline \hline & MSD & MAD & MRD & MARD & RMSD \\
\hline CCSD(T) & 2.9 & 4.2 & 22.0 & 22.9 & 6.1 \\
RPA@HF & 3.5 & 6.2 & 18.1 & 21.1 & 12.0 \\
RPA@PBE & 17.6 & 18.1 & 76.6 & 76.7 & 36.5 \\
\hline \hline
\end{tabular}

Table 32: Statistics for absolute isotropic NMR shieldings in a def2-TZVP basis. CCSD(T) shieldings are used as a reference. $\mathrm{MSD}=$ mean signed deviation; $\mathrm{MAD}=$ mean absolute deviation; $\mathrm{MRD}=$ mean relative deviation [\%]; MARD=mean absolute relative deviation [\%]; RMSD=root mean squared deviation.

\begin{tabular}{lrrrrr}
\hline \hline & MSD & MAD & MRD & MARD & RMSD \\
\hline CCSD(T) & 0.0 & 0.0 & 0.0 & 0.0 & 0.0 \\
RPA@HF & 0.6 & 5.0 & 3.7 & 15.5 & 8.9 \\
RPA@PBE & 14.7 & 15.1 & 48.8 & 49.0 & 32.7 \\
\hline \hline
\end{tabular}


Table 33: Absolute isotropic NMR shieldings in a def2-QZVP basis. All values are given in ppm. "Extrap." stands for basis-set extrapolated CCSD(T)-shieldings in an aug-cc-pCV[TQ]Z basis set. The data for "Extrap." was taken from Ref. 3. CCSD(T) calculations were done with the CFOUR program[1, [2].

\begin{tabular}{|c|c|c|c|c|c|}
\hline Mol. & Nuc. & $\operatorname{CCSD}(\mathrm{T})$ & Extrap. & RPA@HF & RPA@PBE \\
\hline \multirow[t]{2}{*}{$\mathrm{HF}$} & $\mathrm{H}$ & 29.0 & 28.8 & 28.9 & 29.6 \\
\hline & $\mathrm{F}$ & 419.2 & 420.3 & 418.7 & 418.9 \\
\hline \multirow[t]{2}{*}{$\mathrm{CO}$} & $\mathrm{C}$ & 4.4 & 2.2 & -2.2 & 20.8 \\
\hline & $\mathrm{O}$ & -54.9 & -55.0 & -53.9 & -26.9 \\
\hline $\mathrm{N}_{2}$ & $\mathrm{~N}$ & -60.0 & -60.4 & -66.9 & -36.7 \\
\hline \multirow[t]{2}{*}{$\mathrm{H}_{2} \mathrm{O}$} & $\mathrm{O}$ & 337.8 & 338.0 & 335.6 & 336.9 \\
\hline & $\mathrm{H}$ & 30.8 & 30.6 & 30.9 & 31.2 \\
\hline \multirow{3}{*}{$\mathrm{HCN}$} & $\mathrm{H}$ & 29.1 & 29.0 & 29.3 & 29.3 \\
\hline & $\mathrm{C}$ & 86.3 & 84.6 & 82.1 & 93.5 \\
\hline & $\mathrm{N}$ & -12.4 & -14.1 & -17.2 & 7.5 \\
\hline \multirow[t]{3}{*}{$\mathrm{HOF}$} & $\mathrm{O}$ & -63.3 & -68.9 & -61.8 & 5.0 \\
\hline & $\mathrm{H}$ & 19.9 & 19.6 & 20.5 & 22.0 \\
\hline & $\mathrm{F}$ & 190.6 & 192.2 & 213.8 & 211.5 \\
\hline \multirow[t]{2}{*}{$\mathrm{NH}_{3}$} & $\mathrm{~N}$ & 271.1 & 270.7 & 268.0 & 269.5 \\
\hline & $\mathrm{H}$ & 31.6 & 31.4 & 31.7 & 31.8 \\
\hline \multirow[t]{3}{*}{$\mathrm{CH}_{2} \mathrm{O}$} & $\mathrm{O}$ & -378.1 & -378.6 & -351.8 & -272.6 \\
\hline & $\mathrm{C}$ & 4.9 & 1.5 & 4.0 & 23.1 \\
\hline & $\mathrm{H}$ & 22.1 & 22.0 & 22.6 & 23.0 \\
\hline \multirow{2}{*}{$\mathrm{CH}_{4}$} & $\mathrm{C}$ & 199.7 & 198.9 & 196.7 & 197.4 \\
\hline & $\mathrm{H}$ & 31.4 & 31.3 & 31.5 & 31.5 \\
\hline \multirow[t]{2}{*}{$\mathrm{C} 2 \mathrm{H} 4$} & $\mathrm{C}$ & 72.1 & 69.7 & 68.0 & 77.8 \\
\hline & $\mathrm{H}$ & 26.1 & 26.0 & 26.4 & 26.5 \\
\hline \multirow[t]{2}{*}{$\mathrm{AlF}$} & $\mathrm{Al}$ & 572.1 & 572.9 & 575.6 & 579.4 \\
\hline & $\mathrm{F}$ & 211.2 & 211.8 & 227.8 & 227.6 \\
\hline \multirow[t]{3}{*}{$\mathrm{CH}_{3} \mathrm{~F}$} & $\mathrm{C}$ & 124.0 & 122.1 & 124.0 & 126.0 \\
\hline & $\mathrm{F}$ & 482.3 & 482.9 & 479.8 & 474.8 \\
\hline & $\mathrm{H}$ & 27.4 & 27.3 & 27.8 & 27.8 \\
\hline \multirow[t]{4}{*}{$\mathrm{C}_{3} \mathrm{H}_{4}$} & $\mathrm{C}$ & 193.1 & 192.1 & 191.1 & 192.3 \\
\hline & $\mathrm{C}$ & 85.8 & 83.7 & 82.3 & 92.5 \\
\hline & $\mathrm{H}$ & 24.5 & 24.4 & 24.6 & 24.9 \\
\hline & $\mathrm{H}$ & 30.7 & 30.6 & 30.9 & 30.9 \\
\hline $\mathrm{FCCH}$ & C & 181.0 & 179.9 & 178.5 & 181.3 \\
\hline & $\mathrm{C}$ & 101.2 & 100.1 & 100.9 & 104.3 \\
\hline & $\mathrm{H}$ & 30.6 & 30.5 & 30.7 & 30.8 \\
\hline & $\mathrm{F}$ & 423.7 & 423.5 & 424.9 & 422.8 \\
\hline $\mathrm{FCN}$ & $\mathrm{F}$ & 374.7 & 374.1 & 378.7 & 377.6 \\
\hline & $\mathrm{C}$ & 83.3 & 82.2 & 80.5 & 87.4 \\
\hline & $\mathrm{N}$ & 119.4 & 117.9 & 113.2 & 128.0 \\
\hline $\mathrm{H}_{2} \mathrm{~S}$ & $\mathrm{~S}$ & 737.5 & 739.0 & 722.1 & 732.3 \\
\hline & $\mathrm{H}$ & 30.6 & 30.5 & 30.8 & 30.9 \\
\hline HCP & $\mathrm{H}$ & 29.6 & 29.6 & 29.9 & 29.8 \\
\hline & $\mathrm{C}$ & 39.7 & 37.6 & 32.2 & 48.0 \\
\hline & $\mathrm{P}$ & 385.9 & 388.0 & 368.8 & 417.8 \\
\hline $\mathrm{HFCO}$ & $\mathrm{O}$ & -93.1 & -94.3 & -88.1 & -60.8 \\
\hline & $\mathrm{C}$ & 41.7 & 39.6 & 39.6 & 47.3 \\
\hline & $\mathrm{F}$ & 165.2 & 165.3 & 182.0 & 182.2 \\
\hline & $\mathrm{H}$ & 24.0 & 23.9 & 24.3 & 24.3 \\
\hline $\mathrm{H}_{2} \mathrm{C}_{2} \mathrm{O}$ & $\mathrm{C}$ & 194.3 & 193.3 & 191.8 & 193.7 \\
\hline & $\mathrm{C}$ & -4.1 & -6.3 & -6.1 & 4.1 \\
\hline & $\mathrm{O}$ & -6.1 & -5.9 & 1.2 & 15.7 \\
\hline & $\mathrm{H}$ & 29.3 & 29.2 & 29.5 & 29.6 \\
\hline $\mathrm{LiF}$ & $\mathrm{Li}$ & 90.3 & 89.3 & 90.8 & 91.0 \\
\hline & $\mathrm{F}$ & 382.2 & 382.5 & 390.2 & 392.6 \\
\hline $\mathrm{LiH}$ & $\mathrm{H}$ & 26.6 & 26.6 & 26.9 & 26.6 \\
\hline & $\mathrm{Li}$ & 90.0 & 89.3 & 90.2 & 91.3 \\
\hline $\mathrm{N}_{2} \mathrm{O}$ & $\mathrm{N}$ & 108.3 & 106.4 & 100.7 & 119.2 \\
\hline & $\mathrm{N}$ & 13.5 & 12.6 & 4.9 & 26.0 \\
\hline & $\mathrm{O}$ & 199.0 & 199.0 & 198.3 & 207.9 \\
\hline OCS & $\mathrm{O}$ & 97.2 & 96.8 & 98.6 & 111.1 \\
\hline & $\mathrm{C}$ & 31.7 & 30.2 & 25.8 & 40.5 \\
\hline & $\mathrm{S}$ & 798.6 & 796.7 & 798.4 & 806.6 \\
\hline $\mathrm{OF}_{2}$ & $\mathrm{O}$ & -438.0 & -447.1 & -412.7 & -267.5 \\
\hline & $\mathrm{F}$ & -19.3 & -24.0 & 7.6 & 28.3 \\
\hline $\mathrm{H}_{4} \mathrm{C}_{2} \mathrm{O}$ & $\mathrm{O}$ & 363.7 & 363.2 & 365.2 & 359.2 \\
\hline & $\mathrm{C}$ & 154.7 & 153.2 & 154.0 & 155.5 \\
\hline & $\mathrm{H}$ & 29.2 & 29.1 & 29.5 & 29.4 \\
\hline PN & $\mathrm{N}$ & -341.8 & -344.0 & -356.5 & -278.4 \\
\hline & $\mathrm{P}$ & 47.5 & 50.6 & 16.1 & 134.7 \\
\hline
\end{tabular}


Table 34: Statistics for absolute isotropic NMR shieldings in a def2-QZVP basis. CCSD(T)/aug-cc$\mathrm{pCV}[\mathrm{TQ}] \mathrm{Z}$ shieldings are used as a reference. $\mathrm{MSD}=$ mean signed deviation; $\mathrm{MAD}=$ mean absolute deviation; $\mathrm{MRD}=$ mean relative deviation [\%]; $\mathrm{MARD}=$ mean absolute relative deviation [\%]; $\mathrm{RMSD}=$ root mean squared deviation.

\begin{tabular}{lrrrrr}
\hline \hline & MSD & MAD & MRD & MARD & RMSD \\
\hline CCSD(T) & 0.8 & 1.2 & 6.6 & 6.9 & 1.9 \\
RPA@HF & 0.8 & 5.2 & 1.4 & 13.9 & 9.7 \\
RPA@PBE & 14.3 & 15.0 & 59.2 & 59.4 & 32.3 \\
\hline \hline
\end{tabular}

Table 35: Statistics for absolute isotropic NMR shieldings in a def2-QZVP basis. CCSD(T) shieldings are used as a reference. $\mathrm{MSD}=$ mean signed deviation; $\mathrm{MAD}=$ mean absolute deviation; $\mathrm{MRD}=$ mean relative deviation [\%]; MARD=mean absolute relative deviation [\%]; RMSD=root mean squared deviation.

\begin{tabular}{lrrrrr}
\hline \hline & MSD & MAD & MRD & MARD & RMSD \\
\hline CCSD(T) & 0.0 & 0.0 & 0.0 & 0.0 & 0.0 \\
RPA@HF & 0.0 & 5.1 & -2.3 & 12.1 & 9.0 \\
RPA@PBE & 13.5 & 14.2 & 36.8 & 37.0 & 31.2 \\
\hline \hline
\end{tabular}


Table 36: Absolute isotropic NMR shieldings in a pcSseg-0 basis. All values are given in ppm. "Extrap." stands for basis-set extrapolated $\operatorname{CCSD}(\mathrm{T})$-shieldings in an aug-cc-pCV[TQ]Z basis set. The data for "Extrap." was taken from Ref. 3. CCSD(T) calculations were done with the CFOUR program[1, 2.

\begin{tabular}{|c|c|c|c|c|c|}
\hline Mol. & Nuc. & $\overline{C C C S D(T)}$ & Extrap. & RPA@HF & RPA@PBE \\
\hline \multirow[t]{2}{*}{$\mathrm{HF}$} & $\mathrm{H}$ & 30.8 & 28.8 & 30.7 & 30.9 \\
\hline & $\mathrm{F}$ & 430.9 & 420.3 & 432.5 & 432.1 \\
\hline \multirow[t]{2}{*}{$\mathrm{CO}$} & $\mathrm{C}$ & 7.0 & 2.2 & 11.6 & 43.2 \\
\hline & $\mathrm{O}$ & -77.8 & -55.0 & -63.6 & -19.8 \\
\hline $\mathrm{N}_{2}$ & $\mathrm{~N}$ & -74.3 & -60.4 & -63.1 & -25.2 \\
\hline \multirow[t]{2}{*}{$\mathrm{H}_{2} \mathrm{O}$} & $\mathrm{O}$ & 355.8 & 338.0 & 356.3 & 355.3 \\
\hline & $\mathrm{H}$ & 32.8 & 30.6 & 32.8 & 32.8 \\
\hline \multirow[t]{3}{*}{$\mathrm{HCN}$} & $\mathrm{H}$ & 30.4 & 29.0 & 30.5 & 30.5 \\
\hline & $\mathrm{C}$ & 87.6 & 84.6 & 87.1 & 102.5 \\
\hline & $\mathrm{N}$ & -14.7 & -14.1 & -8.9 & 26.5 \\
\hline \multirow[t]{3}{*}{$\mathrm{HOF}$} & $\mathrm{O}$ & -38.8 & -68.9 & -7.3 & 103.6 \\
\hline & $\mathrm{H}$ & 23.6 & 19.6 & 24.4 & 27.0 \\
\hline & $\mathrm{F}$ & 130.4 & 192.2 & 168.0 & 212.2 \\
\hline \multirow[t]{2}{*}{$\mathrm{NH}_{3}$} & $\mathrm{~N}$ & 280.5 & 270.7 & 279.7 & 279.3 \\
\hline & $\mathrm{H}$ & 33.5 & 31.4 & 33.7 & 33.5 \\
\hline \multirow[t]{3}{*}{$\mathrm{CH}_{2} \mathrm{O}$} & $\mathrm{O}$ & -475.5 & -378.6 & -392.7 & -217.6 \\
\hline & $\mathrm{C}$ & 13.6 & 1.5 & 18.8 & 47.9 \\
\hline & $\mathrm{H}$ & 23.1 & 22.0 & 23.7 & 24.8 \\
\hline \multirow[t]{2}{*}{$\mathrm{CH}_{4}$} & $\mathrm{C}$ & 205.6 & 198.9 & 204.9 & 207.2 \\
\hline & $\mathrm{H}$ & 32.7 & 31.3 & 32.8 & 32.6 \\
\hline \multirow[t]{2}{*}{$\mathrm{C} 2 \mathrm{H} 4$} & $\mathrm{C}$ & 82.6 & 69.7 & 78.6 & 92.8 \\
\hline & $\mathrm{H}$ & 27.8 & 26.0 & 27.9 & 28.1 \\
\hline \multirow{2}{*}{$\mathrm{AlF}$} & $\mathrm{Al}$ & 554.5 & 572.9 & 559.3 & 573.8 \\
\hline & $\mathrm{F}$ & 187.8 & 211.8 & 211.7 & 205.1 \\
\hline \multirow[t]{3}{*}{$\mathrm{CH}_{3} \mathrm{~F}$} & $\mathrm{C}$ & 134.9 & 122.1 & 135.4 & 142.1 \\
\hline & $\mathrm{F}$ & 506.2 & 482.9 & 507.0 & 499.3 \\
\hline & $\mathrm{H}$ & 28.9 & 27.3 & 29.1 & 29.1 \\
\hline \multirow[t]{4}{*}{$\mathrm{C}_{3} \mathrm{H}_{4}$} & $\mathrm{C}$ & 207.9 & 192.1 & 207.3 & 210.1 \\
\hline & $\mathrm{C}$ & 96.7 & 83.7 & 96.2 & 108.3 \\
\hline & $\mathrm{H}$ & 27.0 & 24.4 & 27.2 & 27.4 \\
\hline & $\mathrm{H}$ & 32.1 & 30.6 & 32.3 & 32.1 \\
\hline $\mathrm{FCCH}$ & $\mathrm{C}$ & 179.1 & 179.9 & 177.3 & 182.8 \\
\hline & $\mathrm{C}$ & 108.3 & 100.1 & 108.1 & 114.1 \\
\hline & $\mathrm{H}$ & 31.8 & 30.5 & 31.9 & 31.8 \\
\hline & $\mathrm{F}$ & 436.2 & 423.5 & 439.0 & 438.2 \\
\hline $\mathrm{FCN}$ & $\mathrm{F}$ & 391.6 & 374.1 & 400.3 & 402.0 \\
\hline & C & 84.6 & 82.2 & 84.1 & 93.4 \\
\hline & $\mathrm{N}$ & 105.5 & 117.9 & 107.7 & 130.6 \\
\hline $\mathrm{H}_{2} \mathrm{~S}$ & $\mathrm{~S}$ & 789.4 & 739.0 & 797.4 & 806.0 \\
\hline & $\mathrm{H}$ & 32.7 & 30.5 & 32.9 & 32.7 \\
\hline $\mathrm{HCP}$ & $\mathrm{H}$ & 30.9 & 29.6 & 31.1 & 31.1 \\
\hline & C & 39.6 & 37.6 & 37.2 & 58.4 \\
\hline & $\mathrm{P}$ & 405.2 & 388.0 & 404.4 & 469.9 \\
\hline $\mathrm{HFCO}$ & $\mathrm{O}$ & -139.8 & -94.3 & -114.1 & -60.0 \\
\hline & $\mathrm{C}$ & 44.0 & 39.6 & 44.8 & 56.5 \\
\hline & $\mathrm{F}$ & 170.3 & 165.3 & 193.3 & 202.4 \\
\hline & $\mathrm{H}$ & 25.2 & 23.9 & 25.4 & 25.5 \\
\hline $\mathrm{H}_{2} \mathrm{C}_{2} \mathrm{O}$ & $\mathrm{C}$ & 197.7 & 193.3 & 197.2 & 199.8 \\
\hline & $\mathrm{C}$ & -4.4 & -6.3 & 0.9 & 16.0 \\
\hline & $\mathrm{O}$ & -49.1 & -5.9 & -13.0 & 14.2 \\
\hline & $\mathrm{H}$ & 31.2 & 29.2 & 31.4 & 31.3 \\
\hline $\mathrm{LiF}$ & $\mathrm{Li}$ & 89.0 & 89.3 & 89.3 & 89.1 \\
\hline & $\mathrm{F}$ & 301.8 & 382.5 & 318.5 & 302.8 \\
\hline $\mathrm{LiH}$ & $\mathrm{H}$ & 26.1 & 26.6 & 26.2 & 25.7 \\
\hline & $\mathrm{Li}$ & 89.6 & 89.3 & 89.5 & 91.4 \\
\hline $\mathrm{N}_{2} \mathrm{O}$ & $\mathrm{N}$ & 99.0 & 106.4 & 104.3 & 124.2 \\
\hline & $\mathrm{N}$ & 10.0 & 12.6 & 12.2 & 33.9 \\
\hline & $\mathrm{O}$ & 183.1 & 199.0 & 198.1 & 207.1 \\
\hline OCS & $\mathrm{O}$ & 73.4 & 96.8 & 83.4 & 105.0 \\
\hline & $\mathrm{C}$ & 29.1 & 30.2 & 28.7 & 47.1 \\
\hline & $\mathrm{S}$ & 807.2 & 796.7 & 816.8 & 831.3 \\
\hline $\mathrm{OF}_{2}$ & $\mathrm{O}$ & -392.4 & -447.1 & -329.6 & -27.6 \\
\hline & $\mathrm{F}$ & -38.1 & -24.0 & 14.6 & 74.1 \\
\hline $\mathrm{H}_{4} \mathrm{C}_{2} \mathrm{O}$ & $\mathrm{O}$ & 376.6 & 363.2 & 382.4 & 377.3 \\
\hline & $\mathrm{C}$ & 165.1 & 153.2 & 165.6 & 169.9 \\
\hline & $\mathrm{H}$ & 30.8 & 29.1 & 31.0 & 30.8 \\
\hline $\mathrm{PN}$ & $\mathrm{N}$ & -370.9 & -344.0 & -268.3 & -201.9 \\
\hline & $\mathrm{P}$ & 45.1 & 50.6 & 146.9 & 254.6 \\
\hline
\end{tabular}


Table 37: Statistics for absolute isotropic NMR shieldings in a pcSseg-0 basis. CCSD(T)/aug-cc$\mathrm{pCV}[\mathrm{TQ}] \mathrm{Z}$ shieldings are used as a reference. $\mathrm{MSD}=$ mean signed deviation; $\mathrm{MAD}=$ mean absolute deviation; $\mathrm{MRD}=$ mean relative deviation [\%]; $\mathrm{MARD}=$ mean absolute relative deviation [\%]; $\mathrm{RMSD}=$ root mean squared deviation.

\begin{tabular}{lrrrrr}
\hline \hline & MSD & MAD & MRD & MARD & RMSD \\
\hline CCSD(T) & -1.4 & 13.8 & 4.0 & 36.3 & 23.4 \\
RPA@HF & 9.0 & 14.5 & 33.4 & 40.3 & 26.8 \\
RPA@PBE & 30.3 & 32.9 & 118.8 & 119.6 & 70.8 \\
\hline \hline
\end{tabular}

Table 38: Statistics for absolute isotropic NMR shieldings in a pcSseg-0 basis. CCSD(T) shieldings are used as a reference. $\mathrm{MSD}=$ mean signed deviation; $\mathrm{MAD}=$ mean absolute deviation; $\mathrm{MRD}=$ mean relative deviation [\%]; MARD=mean absolute relative deviation [\%]; RMSD=root mean squared deviation.

\begin{tabular}{lrrrrr}
\hline \hline & MSD & MAD & MRD & MARD & RMSD \\
\hline CCSD(T) & 0.0 & 0.0 & 0.0 & 0.0 & 0.0 \\
RPA@HF & 10.5 & 10.9 & 14.9 & 15.4 & 24.7 \\
RPA@PBE & 31.8 & 32.0 & 56.7 & 56.8 & 71.1 \\
\hline \hline
\end{tabular}


Table 39: Testing influence of the auxiliary basis set in a pcSseg-0 basis set. Calculations with the cc-pVDZ/mp2fit, cc-pVTZ/mp2fit, and cc-pVQZ/mp2fit auxiliary basis set are compared. All values are given in $\mathrm{ppm}$.

\begin{tabular}{|c|c|c|c|c|}
\hline Mol. & Nuc. & 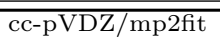 & "cc-pVTZ/mp2fit & $\overline{\text { cc-pVQZ/mp2fit }}$ \\
\hline \multirow[t]{2}{*}{$\mathrm{HF}$} & $\mathrm{H}$ & 30.7 & 30.7 & 30.7 \\
\hline & $\mathrm{F}$ & 432.5 & 432.5 & 432.5 \\
\hline \multirow[t]{2}{*}{$\mathrm{CO}$} & $\mathrm{C}$ & 11.5 & 11.6 & 11.6 \\
\hline & $\mathrm{O}$ & -63.7 & -63.7 & -63.6 \\
\hline $\mathrm{N}_{2}$ & $\mathrm{~N}$ & -63.2 & -63.1 & -63.1 \\
\hline \multirow[t]{2}{*}{$\mathrm{H}_{2} \mathrm{O}$} & $\mathrm{O}$ & 356.3 & 356.3 & 356.3 \\
\hline & $\mathrm{H}$ & 32.8 & 32.8 & 32.8 \\
\hline \multirow[t]{3}{*}{$\mathrm{HCN}$} & $\mathrm{H}$ & 30.5 & 30.5 & 30.5 \\
\hline & $\mathrm{C}$ & 87.1 & 87.1 & 87.1 \\
\hline & $\mathrm{N}$ & -9.0 & -8.9 & -8.9 \\
\hline \multirow[t]{3}{*}{$\mathrm{HOF}$} & $\mathrm{O}$ & -7.6 & -7.4 & -7.3 \\
\hline & $\mathrm{H}$ & 24.4 & 24.4 & 24.4 \\
\hline & $\mathrm{F}$ & 167.9 & 168.0 & 168.0 \\
\hline \multirow[t]{2}{*}{$\mathrm{NH}_{3}$} & $\mathrm{~N}$ & 279.7 & 279.7 & 279.7 \\
\hline & $\mathrm{H}$ & 33.7 & 33.7 & 33.7 \\
\hline \multirow[t]{3}{*}{$\mathrm{CH}_{2} \mathrm{O}$} & $\mathrm{O}$ & -393.1 & -392.7 & -392.7 \\
\hline & $\mathrm{C}$ & 18.8 & 18.8 & 18.8 \\
\hline & $\mathrm{H}$ & 23.7 & 23.7 & 23.7 \\
\hline \multirow[t]{2}{*}{$\mathrm{CH}_{4}$} & $\mathrm{C}$ & 204.8 & 204.8 & 204.9 \\
\hline & $\mathrm{H}$ & 32.8 & 32.8 & 32.8 \\
\hline \multirow[t]{2}{*}{$\mathrm{C} 2 \mathrm{H} 4$} & $\mathrm{C}$ & 78.6 & 78.6 & 78.6 \\
\hline & $\mathrm{H}$ & 27.9 & 27.9 & 27.9 \\
\hline \multirow[t]{2}{*}{$\mathrm{AlF}$} & $\mathrm{Al}$ & 559.2 & 559.3 & 559.3 \\
\hline & $\mathrm{F}$ & 211.6 & 211.7 & 211.7 \\
\hline \multirow[t]{3}{*}{$\mathrm{CH}_{3} \mathrm{~F}$} & $\mathrm{C}$ & 135.4 & 135.4 & 135.4 \\
\hline & $\mathrm{F}$ & 507.0 & 507.0 & 507.0 \\
\hline & $\mathrm{H}$ & 29.1 & 29.1 & 29.1 \\
\hline \multirow[t]{4}{*}{$\mathrm{C}_{3} \mathrm{H}_{4}$} & $\mathrm{C}$ & 207.3 & 207.3 & 207.3 \\
\hline & C & 96.2 & 96.2 & 96.2 \\
\hline & $\mathrm{H}$ & 27.2 & 27.2 & 27.2 \\
\hline & $\mathrm{H}$ & 32.3 & 32.3 & 32.3 \\
\hline $\mathrm{FCCH}$ & $\mathrm{C}$ & 177.3 & 177.3 & 177.3 \\
\hline & $\mathrm{C}$ & 108.1 & 108.1 & 108.1 \\
\hline & $\mathrm{H}$ & 31.9 & 31.9 & 31.9 \\
\hline & $\mathrm{F}$ & 439.0 & 439.0 & 439.0 \\
\hline $\mathrm{FCN}$ & $\mathrm{F}$ & 400.3 & 400.3 & 400.3 \\
\hline & $\mathrm{C}$ & 84.1 & 84.1 & 84.1 \\
\hline & $\mathrm{N}$ & 107.6 & 107.7 & 107.7 \\
\hline $\mathrm{H}_{2} \mathrm{~S}$ & $\mathrm{~S}$ & 797.1 & 797.4 & 797.4 \\
\hline & $\mathrm{H}$ & 32.9 & 32.9 & 32.9 \\
\hline $\mathrm{HCP}$ & $\mathrm{H}$ & 31.1 & 31.1 & 31.1 \\
\hline & $\mathrm{C}$ & 37.1 & 37.2 & 37.2 \\
\hline & $\mathrm{P}$ & 404.2 & 404.4 & 404.4 \\
\hline $\mathrm{HFCO}$ & $\mathrm{O}$ & -114.2 & -114.1 & -114.1 \\
\hline & $\mathrm{C}$ & 44.8 & 44.8 & 44.8 \\
\hline & $\mathrm{F}$ & 193.2 & 193.2 & 193.3 \\
\hline & $\mathrm{H}$ & 25.4 & 25.4 & 25.4 \\
\hline $\mathrm{H}_{2} \mathrm{C}_{2} \mathrm{O}$ & $\mathrm{C}$ & 197.2 & 197.2 & 197.2 \\
\hline & $\mathrm{C}$ & 0.8 & 0.9 & 0.9 \\
\hline & $\mathrm{O}$ & -13.3 & -13.1 & -13.0 \\
\hline & $\mathrm{H}$ & 31.4 & 31.4 & 31.4 \\
\hline $\mathrm{LiF}$ & $\mathrm{Li}$ & 89.3 & 89.3 & 89.3 \\
\hline & $\mathrm{F}$ & 318.5 & 318.5 & 318.5 \\
\hline $\mathrm{LiH}$ & $\mathrm{H}$ & 26.2 & 26.2 & 26.2 \\
\hline & $\mathrm{Li}$ & 89.5 & 89.5 & 89.5 \\
\hline $\mathrm{N}_{2} \mathrm{O}$ & $\mathrm{N}$ & 104.3 & 104.3 & 104.3 \\
\hline & $\mathrm{N}$ & 12.2 & 12.2 & 12.2 \\
\hline & $\mathrm{O}$ & 198.1 & 198.1 & 198.1 \\
\hline OCS & $\mathrm{O}$ & 83.3 & 83.4 & 83.4 \\
\hline & $\mathrm{C}$ & 28.6 & 28.7 & 28.7 \\
\hline & $\mathrm{S}$ & 816.7 & 816.8 & 816.8 \\
\hline $\mathrm{OF}_{2}$ & $\mathrm{O}$ & -330.1 & -329.7 & -329.6 \\
\hline & $\mathrm{F}$ & 14.5 & 14.6 & 14.6 \\
\hline $\mathrm{H}_{4} \mathrm{C}_{2} \mathrm{O}$ & $\mathrm{O}$ & 382.3 & 382.4 & 382.4 \\
\hline & $\mathrm{C}$ & 165.6 & 165.6 & 165.6 \\
\hline & $\mathrm{H}$ & 31.0 & 31.0 & 31.0 \\
\hline $\mathrm{PN}$ & $\mathrm{N}$ & -268.8 & -268.3 & -268.3 \\
\hline & $\mathrm{P}$ & 146.2 & 146.8 & 146.9 \\
\hline
\end{tabular}


Table 40: Absolute isotropic NMR shieldings in a pcSseg-1 basis. All values are given in ppm. "Extrap." stands for basis-set extrapolated $\operatorname{CCSD}(\mathrm{T})$-shieldings in an aug-cc-pCV[TQ]Z basis set. The data for "Extrap." was taken from Ref. 3. CCSD(T) calculations were done with the CFOUR program[1, 2.

\begin{tabular}{|c|c|c|c|c|c|}
\hline Mol. & Nuc. & $\overline{\overline{C C S D}(\mathrm{~T})}$ & Extrap. & 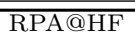 & $\overline{\mathrm{RPA} @ \mathrm{PBE}}$ \\
\hline \multirow[t]{2}{*}{$\mathrm{HF}$} & $\mathrm{H}$ & 29.2 & 28.8 & 29.1 & 29.8 \\
\hline & $\mathrm{F}$ & 419.6 & 420.3 & 419.5 & 418.5 \\
\hline \multirow[t]{2}{*}{$\mathrm{CO}$} & $\mathrm{C}$ & 6.3 & 2.2 & 2.2 & 27.0 \\
\hline & $\mathrm{O}$ & -54.4 & -55.0 & -50.8 & -18.8 \\
\hline $\mathrm{N}_{2}$ & $\mathrm{~N}$ & -58.6 & -60.4 & -61.7 & -29.9 \\
\hline \multirow[t]{2}{*}{$\mathrm{H}_{2} \mathrm{O}$} & $\mathrm{O}$ & 346.3 & 338.0 & 344.4 & 343.8 \\
\hline & $\mathrm{H}$ & 31.1 & 30.6 & 31.1 & 31.4 \\
\hline \multirow[t]{3}{*}{$\mathrm{HCN}$} & $\mathrm{H}$ & 29.2 & 29.0 & 29.4 & 29.5 \\
\hline & $\mathrm{C}$ & 88.0 & 84.6 & 83.9 & 96.0 \\
\hline & $\mathrm{N}$ & -11.6 & -14.1 & -15.1 & 12.8 \\
\hline \multirow[t]{3}{*}{$\mathrm{HOF}$} & $\mathrm{O}$ & -50.7 & -68.9 & -38.9 & 36.3 \\
\hline & $\mathrm{H}$ & 20.7 & 19.6 & 21.4 & 23.2 \\
\hline & $\mathrm{F}$ & 179.2 & 192.2 & 205.2 & 219.3 \\
\hline \multirow[t]{2}{*}{$\mathrm{NH}_{3}$} & $\mathrm{~N}$ & 276.8 & 270.7 & 274.0 & 274.4 \\
\hline & $\mathrm{H}$ & 31.9 & 31.4 & 32.0 & 32.1 \\
\hline \multirow{3}{*}{$\mathrm{CH}_{2} \mathrm{O}$} & $\mathrm{O}$ & -399.5 & -378.6 & -364.8 & -257.5 \\
\hline & $\mathrm{C}$ & 13.0 & 1.5 & 11.9 & 34.0 \\
\hline & $\mathrm{H}$ & 22.6 & 22.0 & 23.0 & 23.7 \\
\hline \multirow[t]{2}{*}{$\mathrm{CH}_{4}$} & $\mathrm{C}$ & 197.9 & 198.9 & 195.7 & 197.0 \\
\hline & $\mathrm{H}$ & 31.4 & 31.3 & 31.5 & 31.5 \\
\hline \multirow[t]{2}{*}{$\mathrm{C} 2 \mathrm{H} 4$} & $\mathrm{C}$ & 74.6 & 69.7 & 69.8 & 81.3 \\
\hline & $\mathrm{H}$ & 26.5 & 26.0 & 26.6 & 26.9 \\
\hline \multirow[t]{2}{*}{$\mathrm{AlF}$} & $\mathrm{Al}$ & 568.6 & 572.9 & 574.1 & 581.9 \\
\hline & $\mathrm{F}$ & 199.6 & 211.8 & 216.7 & 224.4 \\
\hline \multirow[t]{3}{*}{$\mathrm{CH}_{3} \mathrm{~F}$} & $\mathrm{C}$ & 126.0 & 122.1 & 126.0 & 129.9 \\
\hline & $\mathrm{F}$ & 473.8 & 482.9 & 474.3 & 468.8 \\
\hline & $\mathrm{H}$ & 27.6 & 27.3 & 27.9 & 28.0 \\
\hline \multirow[t]{4}{*}{$\mathrm{C}_{3} \mathrm{H}_{4}$} & $\mathrm{C}$ & 193.8 & 192.1 & 192.3 & 194.2 \\
\hline & $\mathrm{C}$ & 88.1 & 83.7 & 84.6 & 95.3 \\
\hline & $\mathrm{H}$ & 24.8 & 24.4 & 25.0 & 25.4 \\
\hline & $\mathrm{H}$ & 31.0 & 30.6 & 31.1 & 31.1 \\
\hline $\mathrm{FCCH}$ & $\mathrm{C}$ & 179.9 & 179.9 & 177.4 & 181.3 \\
\hline & $\mathrm{C}$ & 102.2 & 100.1 & 101.4 & 105.5 \\
\hline & $\mathrm{H}$ & 30.6 & 30.5 & 30.7 & 30.8 \\
\hline & $\mathrm{F}$ & 422.8 & 423.5 & 425.0 & 425.4 \\
\hline $\mathrm{FCN}$ & $\mathrm{F}$ & 374.4 & 374.1 & 380.0 & 380.6 \\
\hline & $\mathrm{C}$ & 84.2 & 82.2 & 81.3 & 88.8 \\
\hline & $\mathrm{N}$ & 119.2 & 117.9 & 114.3 & 131.7 \\
\hline $\mathrm{H}_{2} \mathrm{~S}$ & S & 750.6 & 739.0 & 744.9 & 755.0 \\
\hline & $\mathrm{H}$ & 31.0 & 30.5 & 31.1 & 31.3 \\
\hline $\mathrm{HCP}$ & $\mathrm{H}$ & 29.6 & 29.6 & 29.9 & 29.9 \\
\hline & $\mathrm{C}$ & 44.1 & 37.6 & 35.9 & 54.6 \\
\hline & $\mathrm{P}$ & 399.4 & 388.0 & 383.6 & 441.7 \\
\hline $\mathrm{HFCO}$ & $\mathrm{O}$ & -100.1 & -94.3 & -91.3 & -56.7 \\
\hline & $\mathrm{C}$ & 45.5 & 39.6 & 43.4 & 52.8 \\
\hline & $\mathrm{F}$ & 165.6 & 165.3 & 183.1 & 188.4 \\
\hline & $\mathrm{H}$ & 24.4 & 23.9 & 24.6 & 24.8 \\
\hline $\mathrm{H}_{2} \mathrm{C}_{2} \mathrm{O}$ & $\mathrm{C}$ & 192.4 & 193.3 & 190.6 & 192.2 \\
\hline & $\mathrm{C}$ & -2.5 & -6.3 & -2.9 & 7.5 \\
\hline & $\mathrm{O}$ & -17.3 & -5.9 & -2.5 & 13.9 \\
\hline & $\mathrm{H}$ & 29.5 & 29.2 & 29.7 & 29.8 \\
\hline $\mathrm{LiF}$ & $\mathrm{Li}$ & 90.0 & 89.3 & 90.3 & 90.5 \\
\hline & $\mathrm{F}$ & 345.5 & 382.5 & 355.5 & 351.9 \\
\hline $\mathrm{LiH}$ & $\mathrm{H}$ & 26.5 & 26.6 & 26.7 & 26.5 \\
\hline & $\mathrm{Li}$ & 90.8 & 89.3 & 90.9 & 93.3 \\
\hline $\mathrm{N}_{2} \mathrm{O}$ & $\mathrm{N}$ & 109.6 & 106.4 & 104.7 & 123.5 \\
\hline & $\mathrm{N}$ & 20.5 & 12.6 & 13.5 & 34.6 \\
\hline & $\mathrm{O}$ & 194.2 & 199.0 & 197.8 & 207.7 \\
\hline OCS & $\mathrm{O}$ & 94.7 & 96.8 & 97.3 & 113.2 \\
\hline & $\mathrm{C}$ & 35.0 & 30.2 & 29.5 & 45.0 \\
\hline & $\mathrm{S}$ & 805.4 & 796.7 & 808.9 & 823.4 \\
\hline $\mathrm{OF}_{2}$ & $\mathrm{O}$ & -411.2 & -447.1 & -376.5 & -189.9 \\
\hline & $\mathrm{F}$ & -6.4 & -24.0 & 27.3 & 60.1 \\
\hline $\mathrm{H}_{4} \mathrm{C}_{2} \mathrm{O}$ & $\mathrm{O}$ & 361.3 & 363.2 & 365.7 & 360.7 \\
\hline & $\mathrm{C}$ & 156.7 & 153.2 & 156.4 & 159.1 \\
\hline & $\mathrm{H}$ & 29.5 & 29.1 & 29.7 & $\begin{array}{r}29.7 \\
\end{array}$ \\
\hline $\mathrm{PN}$ & $\mathrm{N}$ & -330.3 & -344.0 & -329.6 & -237.8 \\
\hline & $\mathrm{P}$ & 83.1 & 50.6 & 69.2 & 200.0 \\
\hline
\end{tabular}


Table 41: Statistics for absolute isotropic NMR shieldings in a pcSseg-1 basis. CCSD(T)/aug-cc$\mathrm{pCV}[\mathrm{TQ}] \mathrm{Z}$ shieldings are used as a reference. $\mathrm{MSD}=$ mean signed deviation; $\mathrm{MAD}=$ mean absolute deviation; $\mathrm{MRD}=$ mean relative deviation [\%]; $\mathrm{MARD}=$ mean absolute relative deviation [\%]; $\mathrm{RMSD}=$ root mean squared deviation.

\begin{tabular}{lrrrrr}
\hline \hline & MSD & MAD & MRD & MARD & RMSD \\
\hline CCSD(T) & 1.9 & 5.6 & 17.0 & 24.0 & 9.8 \\
RPA@HF & 3.9 & 5.7 & 17.5 & 18.6 & 12.8 \\
RPA@PBE & 20.4 & 21.9 & 83.1 & 83.5 & 46.7 \\
\hline \hline
\end{tabular}

Table 42: Statistics for absolute isotropic NMR shieldings in a pcSseg-1 basis. CCSD(T) shieldings are used as a reference. $\mathrm{MSD}=$ mean signed deviation; $\mathrm{MAD}=$ mean absolute deviation; $\mathrm{MRD}=$ mean relative deviation [\%]; MARD=mean absolute relative deviation [\%]; RMSD=root mean squared deviation.

\begin{tabular}{lrrrrr}
\hline \hline & MSD & MAD & MRD & MARD & RMSD \\
\hline CCSD(T) & 0.0 & 0.0 & 0.0 & 0.0 & 0.0 \\
RPA@HF & 2.0 & 5.2 & 7.0 & 14.4 & 9.6 \\
RPA@PBE & 18.5 & 18.9 & 47.6 & 47.7 & 41.4 \\
\hline \hline
\end{tabular}


Table 43: Absolute isotropic NMR shieldings in a pcSseg-2 basis. All values are given in ppm. "Extrap." stands for basis-set extrapolated $\operatorname{CCSD}(\mathrm{T})$-shieldings in an aug-cc-pCV[TQ]Z basis set. The data for "Extrap." was taken from Ref. 3, CCSD(T) calculations were done with the CFOUR program[1, 2. MP2 calculations were done with the Turbomole program $4-6$.

\begin{tabular}{|c|c|c|c|c|c|c|c|c|c|c|}
\hline Mol. & Nuc. & $\operatorname{CCSD}(\mathrm{T})$ & Extrap. & RPA@HF & RPA@PBE & RPA@KT2 & RPA@B3LYP & RPA@BH\&H & $\sigma$-P1@PBE & $\overline{M P 2}$ \\
\hline \multirow[t]{2}{*}{$\mathrm{HF}$} & $\mathrm{H}$ & 29.2 & 28.8 & 29.1 & 29.7 & 29.7 & 29.6 & 29.4 & 29.5 & 28.9 \\
\hline & $\mathrm{F}$ & 419.5 & 420.3 & 418.9 & 418.2 & 418.0 & 418.5 & 418.7 & 417.3 & 425.0 \\
\hline \multirow[t]{2}{*}{$\mathrm{CO}$} & $\mathrm{C}$ & 6.5 & 2.2 & 0.6 & 24.7 & 20.4 & 17.4 & 9.8 & 15.3 & 12.1 \\
\hline & $\mathrm{O}$ & -52.3 & -55.0 & -50.7 & -22.6 & -27.0 & -31.3 & -39.5 & -36.2 & -45.4 \\
\hline \multirow{2}{*}{$\mathrm{H}_{2} \mathrm{O}$} & $\mathrm{O}$ & 339.4 & 338.0 & 337.1 & 337.4 & 337.7 & 337.7 & 337.6 & 335.7 & 347.6 \\
\hline & $\mathrm{H}$ & 30.9 & 30.6 & 31.0 & 31.3 & 31.3 & 31.2 & 31.1 & 31.2 & 30.7 \\
\hline \multirow[t]{2}{*}{$\mathrm{HCN}$} & $\mathrm{H}$ & 29.2 & 29.0 & 29.4 & 29.4 & 29.4 & 29.4 & 29.4 & 29.3 & 29.1 \\
\hline & $\mathrm{C}$ & 87.4 & 84.6 & 83.3 & 95.1 & 93.1 & 91.8 & 88.2 & 92.4 & 88.8 \\
\hline \multirow[t]{3}{*}{$\mathrm{HOF}$} & $\mathrm{O}$ & -58.9 & -68.9 & -53.9 & 15.0 & -9.1 & -15.8 & -38.4 & -25.7 & -44.1 \\
\hline & $\mathrm{H}$ & 20.1 & 19.6 & 20.8 & 22.3 & 21.7 & 21.5 & 21.0 & 21.2 & 20.2 \\
\hline & $\mathrm{F}$ & 190.0 & 192.2 & 214.6 & 215.7 & 213.2 & 212.6 & 212.3 & 199.6 & 194.3 \\
\hline \multirow{2}{*}{$\mathrm{NH}_{3}$} & $\mathrm{~N}$ & 272.4 & 270.7 & 269.1 & 270.2 & 270.4 & 270.2 & 269.8 & 269.0 & 278.0 \\
\hline & $\mathrm{H}$ & 31.7 & 31.4 & 31.8 & 31.9 & 31.9 & 31.9 & 31.8 & 31.8 & 31.5 \\
\hline \multirow[t]{3}{*}{$\mathrm{CH}_{2} \mathrm{O}$} & $\mathrm{O}$ & -377.6 & -378.6 & -348.7 & -261.9 & -287.9 & -290.1 & -313.2 & -318.8 & -331.5 \\
\hline & $\mathrm{C}$ & 7.3 & 1.5 & 6.5 & 27.6 & 21.7 & 19.9 & 13.2 & 20.8 & 7.3 \\
\hline & $\mathrm{H}$ & 22.3 & 22.0 & 22.8 & 23.2 & 23.0 & 22.9 & 22.8 & 22.9 & 22.3 \\
\hline \multirow[t]{2}{*}{$\mathrm{CH}_{4}$} & $\mathrm{C}$ & 199.5 & 198.9 & 196.5 & 197.9 & 197.6 & 197.7 & 197.1 & 197.4 & 201.2 \\
\hline & $\mathrm{H}$ & 31.4 & 31.3 & 31.5 & 31.5 & 31.5 & 31.5 & 31.5 & 31.5 & 31.3 \\
\hline $\mathrm{C} 2 \mathrm{H} 4$ & $\mathrm{H}$ & 26.2 & 26.0 & 26.4 & 26.6 & 26.6 & 26.6 & 26.5 & 26.6 & 26.1 \\
\hline $\mathrm{AlF}$ & $\mathrm{Al}$ & 571.0 & 572.9 & 574.7 & 579.8 & 577.1 & 576.7 & 574.2 & 575.6 & 569.6 \\
\hline & $\mathrm{F}$ & 213.9 & 211.8 & 229.1 & 239.0 & 236.4 & 233.0 & 229.3 & 242.6 & 210.3 \\
\hline $\mathrm{CH}_{3} \mathrm{~F}$ & $\mathrm{C}$ & 124.2 & 122.1 & 124.3 & 127.9 & 126.9 & 126.8 & 125.1 & 127.2 & 122.7 \\
\hline & $\mathrm{F}$ & 479.4 & 482.9 & 477.4 & 472.0 & 472.4 & 473.3 & 474.7 & 474.1 & 485.9 \\
\hline & $\mathrm{H}$ & 27.5 & 27.3 & 27.9 & 27.9 & 27.9 & 27.8 & 27.8 & 27.9 & 27.4 \\
\hline $\mathrm{C}_{3} \mathrm{H}_{4}$ & $\mathrm{C}$ & 193.2 & 192.1 & 191.4 & 192.9 & 192.2 & 192.4 & 191.7 & 192.8 & 194.3 \\
\hline & $\mathrm{C}$ & 85.9 & 83.7 & 82.5 & 92.7 & 90.7 & 89.9 & 86.5 & 90.7 & 87.2 \\
\hline & $\mathrm{H}$ & 24.6 & 24.4 & 24.7 & 25.1 & 25.1 & 25.0 & 24.9 & 25.0 & 24.5 \\
\hline & $\mathrm{H}$ & 30.8 & 30.6 & 31.0 & 31.0 & 31.0 & 31.0 & 31.0 & 31.0 & 30.8 \\
\hline $\mathrm{FCCH}$ & $\mathrm{C}$ & 181.4 & 179.9 & 179.0 & 182.4 & 181.2 & 182.1 & 181.0 & 182.1 & 183.1 \\
\hline & $\mathrm{C}$ & 101.8 & 100.1 & 101.5 & 106.0 & 105.0 & 104.9 & 103.2 & 106.0 & 99.1 \\
\hline & $\mathrm{H}$ & 30.6 & 30.5 & 30.8 & 30.8 & 30.9 & 30.9 & 30.8 & 30.8 & 30.6 \\
\hline & $\mathrm{F}$ & 424.5 & 423.5 & 425.8 & 425.5 & 425.0 & 425.3 & 425.5 & 425.9 & 430.7 \\
\hline $\mathrm{FCN}$ & $\mathrm{F}$ & 375.2 & 374.1 & 379.2 & 378.9 & 379.1 & 378.0 & 378.2 & 374.7 & 384.1 \\
\hline & $\mathrm{C}$ & 84.3 & 82.2 & 81.6 & 89.3 & 88.0 & 87.8 & 85.1 & 88.4 & 83.2 \\
\hline & $\mathrm{N}$ & 120.1 & 117.9 & 114.6 & 130.5 & 127.3 & 128.0 & 123.4 & 126.7 & 133.4 \\
\hline $\mathrm{H}_{2} \mathrm{~S}$ & $\mathrm{~S}$ & 732.1 & 739.0 & 718.0 & 725.7 & 724.2 & 723.2 & 720.2 & 712.9 & 748.4 \\
\hline & $\mathrm{H}$ & 30.7 & 30.5 & 30.9 & 31.1 & 31.1 & 31.1 & 31.0 & 31.1 & 30.5 \\
\hline $\mathrm{HFCO}$ & $\mathrm{O}$ & -93.0 & -94.3 & -86.9 & -57.6 & -61.8 & -62.3 & -69.5 & -76.0 & -61.3 \\
\hline & $\mathrm{C}$ & 43.2 & 39.6 & 41.1 & 50.6 & 48.9 & 48.4 & 45.1 & 47.9 & 43.4 \\
\hline & $\mathrm{F}$ & 166.8 & 165.3 & 183.3 & 186.5 & 182.2 & 184.1 & 181.9 & 179.5 & 166.5 \\
\hline & $\mathrm{H}$ & 24.1 & 23.9 & 24.4 & 24.4 & 24.4 & 24.4 & 24.4 & 24.4 & 24.1 \\
\hline $\mathrm{H}_{2} \mathrm{C}_{2} \mathrm{O}$ & $\mathrm{C}$ & 194.4 & 193.3 & 192.0 & 193.8 & 193.5 & 193.4 & 192.9 & 192.5 & 199.3 \\
\hline & $\mathrm{C}$ & -3.3 & -6.3 & -4.8 & 5.9 & 4.8 & 3.5 & 0.1 & -0.5 & 0.7 \\
\hline & $\mathrm{O}$ & -6.7 & -5.9 & 2.2 & 16.8 & 16.1 & 14.9 & 11.8 & 4.0 & 28.4 \\
\hline & $\mathrm{H}$ & 29.3 & 29.2 & 29.5 & 29.6 & 29.7 & 29.6 & 29.6 & 29.5 & 29.4 \\
\hline $\mathrm{LiF}$ & $\mathrm{Li}$ & 89.6 & 89.3 & 90.0 & 90.2 & 89.6 & 90.0 & 89.8 & 90.5 & 89.5 \\
\hline & $\mathrm{F}$ & 372.0 & 382.5 & 380.9 & 382.9 & 374.3 & 382.1 & 380.3 & 390.5 & 369.7 \\
\hline $\mathrm{LiH}$ & $\mathrm{H}$ & 26.6 & 26.6 & 26.9 & 26.6 & 26.6 & 26.7 & 26.8 & 26.8 & 26.7 \\
\hline & $\mathrm{Li}$ & 89.4 & 89.3 & 89.5 & 91.1 & 90.6 & 90.5 & 90.0 & 91.2 & 89.4 \\
\hline $\mathrm{N}_{2} \mathrm{O}$ & $\mathrm{N}$ & 109.6 & 106.4 & 103.0 & 121.7 & 119.7 & 117.5 & 111.9 & 115.2 & 132.7 \\
\hline & $\mathrm{N}$ & 15.3 & 12.6 & 7.2 & 28.4 & 26.7 & 23.2 & 16.8 & 24.3 & 33.5 \\
\hline & $\mathrm{O}$ & 200.8 & 199.0 & 200.9 & 210.8 & 210.6 & 208.8 & 206.2 & 202.8 & 218.9 \\
\hline OCS & $\mathrm{O}$ & 99.9 & 96.8 & 101.1 & 115.3 & 114.1 & 111.9 & 107.7 & 111.2 & 113.0 \\
\hline & $\mathrm{C}$ & 32.9 & 30.2 & 27.0 & 42.6 & 41.2 & 39.1 & 34.3 & 40.4 & 39.9 \\
\hline & $\mathrm{S}$ & 797.4 & 796.7 & 796.6 & 806.1 & 804.3 & 803.2 & 800.3 & 794.5 & 816.8 \\
\hline $\mathrm{OF}_{2}$ & $\mathrm{O}$ & -429.7 & -447.1 & -401.2 & -245.5 & -317.2 & -328.9 & -378.8 & -349.4 & -452.3 \\
\hline & $\mathrm{F}$ & -16.9 & -24.0 & 12.9 & 36.9 & 30.3 & 26.0 & 17.9 & 7.5 & -7.0 \\
\hline $\mathrm{H}_{4} \mathrm{C}_{2} \mathrm{O}$ & $\mathrm{O}$ & 363.6 & 363.2 & 365.5 & 360.5 & 359.5 & 362.7 & 364.1 & 360.3 & 372.5 \\
\hline & $\mathrm{C}$ & 154.9 & 153.2 & 154.4 & 157.0 & 156.0 & 156.2 & 155.0 & 156.2 & 154.2 \\
\hline & $\mathrm{H}$ & 29.3 & 29.1 & 29.6 & 29.5 & 29.5 & 29.5 & 29.5 & 29.5 & 29.2 \\
\hline $\mathrm{PN}$ & $\mathrm{N}$ & -339.8 & -344.0 & -350.0 & -262.9 & -281.1 & -290.5 & -317.0 & -289.4 & -248.0 \\
\hline & $\mathrm{P}$ & 42.7 & 50.6 & 10.8 & 129.4 & 105.3 & 87.9 & 51.8 & 82.7 & 104.9 \\
\hline
\end{tabular}


Table 44: Absolute isotropic NMR shieldings in a pcSseg-2 basis. All values are given in ppm. "Extrap." stands for basis-set extrapolated $\operatorname{CCSD}(\mathrm{T})$-shieldings in an aug-cc-pCV[TQ]Z basis set. The data for "Extrap." was taken from Ref. 3. CCSD(T) calculations were done with the CFOUR program[1, 2.

\begin{tabular}{|c|c|c|c|c|c|c|c|}
\hline Mol. & Nuc. & $\overline{\mathrm{CCSD}(\mathrm{T})}$ & Extrap. & HF(num.) & PBE(num.) & KT2(num.) & $\overline{\text { B3LYP(num.) }}$ \\
\hline \multirow[t]{2}{*}{$\mathrm{HF}$} & $\mathrm{H}$ & 29.2 & 28.8 & 28.3 & 29.9 & 29.9 & 29.4 \\
\hline & $\mathrm{F}$ & 419.5 & 420.3 & 414.5 & 411.0 & 408.6 & 411.3 \\
\hline \multirow[t]{2}{*}{$\mathrm{CO}$} & $\mathrm{C}$ & 6.5 & 2.2 & -27.0 & -17.2 & -0.9 & -22.8 \\
\hline & $\mathrm{O}$ & -52.3 & -55.0 & -91.8 & -85.7 & -68.1 & -88.6 \\
\hline $\mathrm{N}_{2}$ & $\mathrm{~N}$ & -56.7 & -60.4 & -114.9 & -88.8 & -69.1 & -97.2 \\
\hline \multirow[t]{2}{*}{$\mathrm{H}_{2} \mathrm{O}$} & $\mathrm{O}$ & 339.4 & 338.0 & 328.8 & 330.4 & 326.9 & 328.4 \\
\hline & $\mathrm{H}$ & 30.9 & 30.6 & 30.6 & 31.3 & 31.4 & 31.1 \\
\hline \multirow[t]{3}{*}{$\mathrm{HCN}$} & $\mathrm{H}$ & 29.2 & 29.0 & 29.3 & 29.2 & 28.9 & 29.3 \\
\hline & $\mathrm{C}$ & 87.4 & 84.6 & 70.3 & 69.6 & 80.2 & 67.1 \\
\hline & $\mathrm{N}$ & -11.3 & -14.1 & -53.2 & -49.2 & -28.0 & -54.2 \\
\hline \multirow{3}{*}{$\mathrm{HOF}$} & $\mathrm{O}$ & -58.9 & -68.9 & -135.9 & -129.4 & -97.8 & -138.2 \\
\hline & $\mathrm{H}$ & 20.1 & 19.6 & 19.2 & 19.5 & 20.2 & 19.5 \\
\hline & $\mathrm{F}$ & 190.0 & 192.2 & 287.0 & 147.2 & 156.8 & 172.3 \\
\hline \multirow{2}{*}{$\mathrm{NH}_{3}$} & $\mathrm{~N}$ & 272.4 & 270.7 & 263.5 & 264.1 & 263.1 & 261.6 \\
\hline & $\mathrm{H}$ & 31.7 & 31.4 & 31.7 & 31.9 & 31.9 & 31.8 \\
\hline \multirow[t]{3}{*}{$\mathrm{CH}_{2} \mathrm{O}$} & $\mathrm{O}$ & -377.6 & -378.6 & -449.6 & -461.8 & -398.7 & -466.7 \\
\hline & $\mathrm{C}$ & 7.3 & 1.5 & -7.8 & -28.6 & -11.1 & -26.9 \\
\hline & $\mathrm{H}$ & 22.3 & 22.0 & 22.6 & 20.9 & 21.5 & 21.5 \\
\hline \multirow[t]{2}{*}{$\mathrm{CH}_{4}$} & $\mathrm{C}$ & 199.5 & 198.9 & 195.1 & 190.1 & 192.0 & 188.4 \\
\hline & $\mathrm{H}$ & 31.4 & 31.3 & 31.6 & 31.5 & 31.5 & 31.5 \\
\hline \multirow[t]{2}{*}{$\mathrm{C} 2 \mathrm{H} 4$} & $\mathrm{C}$ & 72.7 & 69.7 & 58.9 & 46.6 & 58.2 & 45.8 \\
\hline & $\mathrm{H}$ & 26.2 & 26.0 & 26.2 & 25.6 & 25.9 & 25.9 \\
\hline \multirow[t]{2}{*}{$\mathrm{AlF}$} & $\mathrm{Al}$ & 571.0 & 572.9 & 577.2 & 540.4 & 556.7 & 547.2 \\
\hline & $\mathrm{F}$ & 213.9 & 211.8 & 226.2 & 140.6 & 140.7 & 164.3 \\
\hline \multirow[t]{3}{*}{$\mathrm{CH}_{3} \mathrm{~F}$} & $\mathrm{C}$ & 124.2 & 122.1 & 125.1 & 104.5 & 109.7 & 105.8 \\
\hline & $\mathrm{F}$ & 479.4 & 482.9 & 485.7 & 459.8 & 458.0 & 464.7 \\
\hline & $\mathrm{H}$ & 27.5 & 27.3 & 28.0 & 27.1 & 27.2 & 27.4 \\
\hline $\mathrm{C}_{3} \mathrm{H}_{4}$ & $\mathrm{C}$ & 193.2 & 192.1 & 193.6 & 176.7 & 180.0 & 177.9 \\
\hline & $\mathrm{C}$ & 85.9 & 83.7 & 70.3 & 61.3 & 71.8 & 59.6 \\
\hline & $\mathrm{H}$ & 24.6 & 24.4 & 24.1 & 24.2 & 24.3 & 24.3 \\
\hline & $\mathrm{H}$ & 30.8 & 30.6 & 30.9 & 30.6 & 30.6 & 30.7 \\
\hline $\mathrm{FCCH}$ & $\mathrm{C}$ & 181.4 & 179.9 & 176.1 & 169.5 & 177.4 & 168.7 \\
\hline & $\mathrm{C}$ & 101.8 & 100.1 & 100.5 & 81.5 & 89.5 & 82.2 \\
\hline & $\mathrm{H}$ & 30.6 & 30.5 & 30.5 & 31.0 & 30.8 & 30.9 \\
\hline & $\mathrm{F}$ & 424.5 & 423.5 & 428.3 & 388.4 & 391.1 & 399.9 \\
\hline $\mathrm{FCN}$ & $\mathrm{F}$ & 375.2 & 374.1 & 377.3 & 338.2 & 334.4 & 350.6 \\
\hline & $\mathrm{C}$ & 84.3 & 82.2 & 74.9 & 67.8 & 77.4 & 65.1 \\
\hline & $\mathrm{N}$ & 120.1 & 117.9 & 90.1 & 97.4 & 113.8 & 90.4 \\
\hline $\mathrm{H}_{2} \mathrm{~S}$ & $\mathrm{~S}$ & 732.1 & 739.0 & 712.4 & 710.4 & 725.8 & 694.4 \\
\hline & $\mathrm{H}$ & 30.7 & 30.5 & 30.6 & 30.8 & 30.8 & 30.8 \\
\hline $\mathrm{HCP}$ & $\mathrm{H}$ & 29.7 & 29.6 & 30.1 & 29.6 & 29.2 & 29.8 \\
\hline & $\mathrm{C}$ & 41.5 & 37.6 & 11.6 & 11.5 & 23.1 & 7.1 \\
\hline & $\mathrm{P}$ & 381.7 & 388.0 & 330.5 & 320.1 & 375.4 & 310.0 \\
\hline $\mathrm{HFCO}$ & $\mathrm{O}$ & -93.0 & -94.3 & -134.0 & -132.2 & -107.2 & -141.5 \\
\hline & $\mathrm{C}$ & 43.2 & 39.6 & 33.4 & 20.3 & 30.6 & 19.0 \\
\hline & $\mathrm{F}$ & 166.8 & 165.3 & 186.3 & 88.2 & 101.2 & 112.9 \\
\hline & $\mathrm{H}$ & 24.1 & 23.9 & 24.5 & 23.4 & 23.5 & 23.8 \\
\hline $\mathrm{H}_{2} \mathrm{C}_{2} \mathrm{O}$ & $\mathrm{C}$ & 194.4 & 193.3 & 189.4 & 185.0 & 188.7 & 183.3 \\
\hline & $\mathrm{C}$ & -3.3 & -6.3 & -15.1 & -19.7 & -8.6 & -25.3 \\
\hline & $\mathrm{O}$ & -6.7 & -5.9 & -30.9 & -25.5 & -11.8 & -33.5 \\
\hline & $\mathrm{H}$ & 29.3 & 29.2 & 29.3 & 29.3 & 29.3 & 29.4 \\
\hline $\mathrm{LiF}$ & $\mathrm{Li}$ & 89.6 & 89.3 & 90.8 & 86.7 & 90.6 & 87.7 \\
\hline & $\mathrm{F}$ & 372.0 & 382.5 & 383.2 & 323.1 & 345.8 & 335.6 \\
\hline $\mathrm{LiH}$ & $\mathrm{H}$ & 26.6 & 26.6 & 26.5 & 26.2 & 26.4 & 26.5 \\
\hline & $\mathrm{Li}$ & 89.4 & 89.3 & 89.5 & 88.1 & 92.0 & 88.2 \\
\hline $\mathrm{N}_{2} \mathrm{O}$ & $\mathrm{N}$ & 109.6 & 106.4 & 61.7 & 89.6 & 96.2 & 79.4 \\
\hline & $\mathrm{N}$ & 15.3 & 12.6 & -34.8 & -0.3 & 6.5 & -13.6 \\
\hline & $\mathrm{O}$ & 200.8 & 199.0 & 173.2 & 173.7 & 171.5 & 170.8 \\
\hline OCS & $\mathrm{O}$ & 99.9 & 96.8 & 73.9 & 68.3 & 75.4 & 68.2 \\
\hline & $\mathrm{C}$ & 32.9 & 30.2 & 6.8 & 21.8 & 30.6 & 13.1 \\
\hline & $\mathrm{S}$ & 797.4 & 796.7 & 784.1 & 762.1 & 778.4 & 759.2 \\
\hline $\mathrm{OF}_{2}$ & $\mathrm{O}$ & -429.7 & -447.1 & -444.5 & -631.8 & -548.1 & -590.8 \\
\hline & $\mathrm{F}$ & -16.9 & -24.0 & 22.4 & -89.8 & -71.5 & -72.5 \\
\hline $\mathrm{H}_{4} \mathrm{C}_{2} \mathrm{O}$ & $\mathrm{O}$ & 363.6 & 363.2 & 378.7 & 331.9 & 336.0 & 339.0 \\
\hline & $\mathrm{C}$ & 154.9 & 153.2 & 155.7 & 137.1 & 142.3 & 138.0 \\
\hline & $\mathrm{H}$ & 29.3 & 29.1 & 29.7 & 29.1 & 29.2 & 29.3 \\
\hline $\mathrm{PN}$ & $\mathrm{N}$ & -339.8 & -344.0 & -521.1 & -426.6 & -390.3 & -454.9 \\
\hline & $\mathrm{P}$ & 42.7 & 50.6 & -127.4 & -54.9 & 27.0 & -81.9 \\
\hline
\end{tabular}


Table 45: Statistics for absolute isotropic NMR shieldings in a pcSseg-2 basis. CCSD(T)/aug-cc$\mathrm{pCV}[\mathrm{TQ}] \mathrm{Z}$ shieldings are used as a reference. $\mathrm{MSD}=$ mean signed deviation; $\mathrm{MAD}=\mathrm{mean}$ absolute deviation; $\mathrm{MRD}=$ mean relative deviation [\%]; $\mathrm{MARD}=$ mean absolute relative deviation [\%]; $\mathrm{RMSD}=\mathrm{root}$ mean squared deviation.

\begin{tabular}{lrrrrr}
\hline & MSD & MAD & MRD & MARD & RMSD \\
\hline HF(num.) & -13.4 & 20.0 & -58.1 & 66.7 & 39.5 \\
PBE(num.) & -24.9 & 25.0 & -75.9 & 76.3 & 39.9 \\
KT2(num.) & -13.8 & 14.1 & -29.0 & 29.6 & 23.3 \\
B3LYP(num.) & -25.1 & 25.2 & -83.9 & 84.3 & 38.9 \\
CCSD & 0.4 & 3.9 & 3.1 & 11.0 & 8.4 \\
CCSD(T) & 1.2 & 2.4 & 11.3 & 12.4 & 3.8 \\
RPA@HF & 1.6 & 5.6 & 7.8 & 15.3 & 11.4 \\
RPA@BH\&H & 6.5 & 7.7 & 31.0 & 31.3 & 15.2 \\
RPA@B3LYP & 11.1 & 11.9 & 50.1 & 50.3 & 23.5 \\
RPA@KT2 & 12.3 & 13.5 & 56.5 & 56.8 & 26.1 \\
RPA@PBE & 16.2 & 17.1 & 69.5 & 69.7 & 36.0 \\
$\sigma-P 1 @ P B E$ & 8.9 & 10.3 & 43.8 & 44.1 & 19.3 \\
MP2 & 8.6 & 9.3 & 34.2 & 34.4 & 18.0 \\
\hline \hline
\end{tabular}

Table 46: Statistics for absolute isotropic NMR shieldings in a pcSseg-2 basis. CCSD(T) shieldings are used as a reference. $\mathrm{MSD}=$ mean signed deviation; $\mathrm{MAD}=$ mean absolute deviation; $\mathrm{MRD}=$ mean relative deviation [\%]; MARD=mean absolute relative deviation [\%]; RMSD=root mean squared deviation.

\begin{tabular}{lrrrrr}
\hline \hline & MSD & MAD & MRD & MARD & RMSD \\
\hline HF(num.) & -14.6 & 21.0 & -44.7 & 54.2 & 40.0 \\
PBE(num.) & -26.1 & 26.1 & -54.9 & 55.0 & 41.6 \\
KT2(num.) & -15.0 & 15.2 & -25.5 & 25.8 & 25.2 \\
B3LYP(num.) & -26.2 & 26.3 & -62.2 & 62.3 & 40.4 \\
CCSD & -0.8 & 3.4 & -4.8 & 8.1 & 6.4 \\
CCSD(T) & 0.0 & 0.0 & 0.0 & 0.0 & 0.0 \\
RPA@HF & 0.4 & 5.2 & -0.2 & 11.8 & 9.4 \\
RPA@BH\&H & 5.4 & 6.2 & 15.0 & 15.2 & 13.0 \\
RPA@B3LYP & 9.9 & 10.6 & 26.6 & 26.8 & 21.3 \\
RPA@KT2 & 11.1 & 11.9 & 30.8 & 31.0 & 23.9 \\
RPA@PBE & 15.0 & 15.7 & 38.0 & 38.1 & 33.9 \\
$\sigma$-P1@PBE & 7.7 & 9.0 & 19.4 & 19.7 & 17.1 \\
MP2 & 7.4 & 8.6 & 20.7 & 21.4 & 17.5 \\
\hline \hline
\end{tabular}




\section{References}

[1] D. A. Matthews, L. Cheng, M. E. Harding, F. Lipparini, S. Stopkowicz, T.-C. Jagau, P. G. Szalay, J. Gauss, and J. F. Stanton, J. Chem. Phys. 152, 214108 (2020).

[2] J. F. Stanton, J. Gauss, L. Cheng, M. E. Harding, D. A. Matthews, and P. G. Szalay, "CFOUR, Coupled-Cluster techniques for Computational Chemistry, a quantum-chemical program package," With contributions from A.A. Auer, R.J. Bartlett, U. Benedikt, C. Berger, D.E. Bernholdt, S. Blaschke, Y. J. Bomble, S. Burger, O. Christiansen, D. Datta, F. Engel, R. Faber, J. Greiner, M. Heckert, O. Heun, M. Hilgenberg, C. Huber, T.-C. Jagau, D. Jonsson, J. Jusélius, T. Kirsch, K. Klein, G.M. KopperW.J. Lauderdale, F. Lipparini, T. Metzroth, L.A. Mück, D.P. O'Neill, T. Nottoli, D.R. Price, E. Prochnow, C. Puzzarini, K. Ruud, F. Schiffmann, W. Schwalbach, C. Simmons, S. Stopkowicz, A. Tajti, J. Vázquez, F. Wang, J.D. Watts and the integral packages MOLECULE (J. Almlöf and P.R. Taylor), PROPS (P.R. Taylor), ABACUS (T. Helgaker, H.J. Aa. Jensen, P. Jørgensen, and J. Olsen), and ECP routines by A. V. Mitin and C. van Wüllen. For the current version, see http://www.cfour.de.

[3] A. M. Teale, O. B. Lutnæs, T. Helgaker, D. J. Tozer, and J. Gauss, J. Chem. Phys. 138, 024111 (2013).

[4] "TURBOMOLE V7.4 2019, a development of University of Karlsruhe and Forschungszentrum Karlsruhe GmbH, 1989-2007, TURBOMOLE GmbH, since 2007; available from http://www.turbomole.com." .

[5] M. Kollwitz and J. Gauss, Chem. Phys. Lett. 260, 639 (1996).

[6] M. Kollwitz, M. Häser, and J. Gauss, J. Chem. Phys. 108, 8295 (1998). 